\title{
Formal Total Synthesis of Oximidine II via a Suzuki-Type Cross-Coupling Macrocyclization Employing Potassium Organotrifluoroborates
}

\author{
Gary A. Molander* and Florian Dehmel
}

General Information. Proton NMR spectra were recorded on Bruker AMX (500 MHz) or Bruker DMX $(500 \mathrm{MHz})$ spectrometers. Proton chemical shifts are reported in ppm $(\delta)$ relative to the solvent reference relative to TMS employed as the internal standard $\left(\mathrm{CDCl}_{3}, \delta 7.24 \mathrm{ppm}\right.$; acetone- $\left.d_{6}, \delta 2.05 \mathrm{ppm}\right)$. Data are reported as follows: chemical shift (multiplicity [singlet (s), doublet $(\mathrm{d})$, triplet $(\mathrm{t})$, quartet $(\mathrm{q})$, and multiplet (m)], coupling constants [Hz], integration). Carbon NMR spectra were recorded on Bruker AMX $(125 \mathrm{MHz})$ or Bruker DRX (125 MHz) spectrometers with complete proton decoupling. Carbon chemical shifts are reported in ppm $(\delta)$ relative to solvent resonance as the internal standard $\left(\mathrm{CDCl}_{3}, \delta 77.0\right.$; acetone$\left.d_{6}, \delta 29.8 \mathrm{ppm}, \mathrm{DMSO}-d_{6}, \delta 39.5 \mathrm{ppm}\right)$. NMR data were collected at $25^{\circ} \mathrm{C}$.

Infrared spectra were obtained on Perkin Elmer FT-IR 1600 spectrometer.

Melting points were recorded on a Thomas-Hoover capillary melting point apparatus and are not corrected. Optical rotations were recorded on a Jasco Polarimeter at room temperature (path length $100 \mathrm{~mm}$ ).

Mass spectra were obtained on Micromass autospec in Electrospray Ionization (EI) and Chemical Ionization (CI) modes using the OPUS software system. The method of ionization is given in parentheses. All reactions were run using standard bench conditions. If moisture or oxygen sensitive reagents were used, the experiments were conducted using Schlenk techniques under a $\mathrm{N}_{2}$-atomsphere. THF, toluene and $\mathrm{Et}_{2} \mathrm{O}$ were dried over $\mathrm{Na}$ /benzophenone and distilled, $\mathrm{CH}_{2} \mathrm{Cl}_{2}$ and $\mathrm{NEt}_{3}$ was dried over $\mathrm{CaH}_{2}$ and distilled prior to use. Other solvent were -if necessary- dried with 3 or $4 \AA$ molecular sieves. All reagents were used as received from commercial sources. $(2 R, 4 S)$-2-Phenyl-[1,3]dioxane-4-carbaldehyde 12 was prepared according to literature procedures. ${ }^{1}$

Chromatographical purification was performed using either column chromatography (silica gel or alumina), or MPLC (Biotage ${ }^{\circledR}-$ system), or preparative rotating TLC ("Chromatotron", Harrison Research).

(Z)-1-Triisopropylsilyl-6-trimethylsilyl-hex-1,5-diyn-3-ene (16). $380 \mathrm{mg}$ of $\left(\mathrm{Ph}_{3} \mathrm{P}\right)_{4} \mathrm{Pd}$ $(0.3 \mathrm{mmol}, 3 \mathrm{~mol} \%)$ and $60 \mathrm{mg}$ of $\mathrm{CuI}(0.3 \mathrm{mmol}, 3 \mathrm{~mol} \%)$ were suspended in $50 \mathrm{~mL}$ of $\mathrm{Et}_{2} \mathrm{O} .2 .20 \mathrm{~mL}$ of $n-$ $\mathrm{BuNH}_{2}$ (22.0 mmol, 2.0 equiv), $1.74 \mathrm{~mL}$ of (Z)-1,2-dichloroethylene 15 (21.0 mmol, 1.9 equiv) and $2.55 \mathrm{~mL}$ of triisopropylsilylacetylene $(11.0 \mathrm{mmol})$ was added and the reaction mixture was stirred at rt overnight. After evaporation of the solvent, the residue was taken up with petroleum ether and filtered through a silica/celite pad. Flash-chromatography (silica, petroleum ether 100\%) yielded a colourless oil (2.48 g, $10.2 \mathrm{mmol}, 93 \%) . \mathrm{R}_{f}$ (petroleum ether) $=0.77 .{ }^{1} \mathrm{H}$ NMR $\left(500 \mathrm{MHz}, \mathrm{CDCl}_{3}\right): \delta[\mathrm{ppm}]=1.07(\mathrm{~s}, 3 \mathrm{H}), 1.09$ $(\mathrm{s}, 18 \mathrm{H}), 5.89(\mathrm{~d}, J=7.5 \mathrm{~Hz}, 1 \mathrm{H}), 6.39(\mathrm{~d}, J=7.5 \mathrm{~Hz}, 1 \mathrm{H}) .{ }^{13} \mathrm{C} \mathrm{NMR}\left(125 \mathrm{MHz}, \mathrm{CDCl}_{3}\right): \delta[\mathrm{ppm}]=11.3$, 18.6, 100.1, 100.5, 112.4, 129.2 .

same as lit. ${ }^{2}$

[1] (a) Isobe, M.; Ychikawa, Y.; Bai, D.-1.; Goto, T. Tetrahedron Lett. 1985, 26, 5203; (b) Pawlak, J.; Nakanishi, K.; Iwashita, T.; Borowski, E. J. Org. Chem. 1987, 52, 2896.

$\left.{ }^{2}\right]$ Lu, Y.-F.; Harwig, C. W.; Fallis, A. G. Can. J. Chem. 1995, 73, 2253. 
To a suspension of $140 \mathrm{mg}$ of $\left(\mathrm{Ph}_{3} \mathrm{P}\right)_{4} \mathrm{Pd}(0.12 \mathrm{mmol}, 2.5 \mathrm{~mol} \%), 25 \mathrm{mg}$ of $\mathrm{CuI}(0.13 \mathrm{mmol}, 2.5 \mathrm{~mol} \%)$, $1.40 \mathrm{~mL}$ of trimethylsilylacetylene (9.9 mmol, 2.0 equiv) and $1.20 \mathrm{~g}$ of crude chloroene-yne (4.9 mmol) in $25 \mathrm{~mL}$ of $\mathrm{Et}_{2} \mathrm{O}$ and $1.0 \mathrm{~mL}$ of $n-\mathrm{BuNH}_{2}(10.0 \mathrm{mmol}, 2.0$ equiv) was added and the reaction mixture was stirred at rt overnight. After evaporation of the solvent, the residue was taken up with petroleum ether and filtered through a silica/Celite pad. Flash chromatography (silica, petroleum ether 100\%) yielded a colourless oil (1.44 g, $4.7 \mathrm{mmol}, 96 \%) . \mathrm{R}_{f}$ (petroleum ether) $=0.48 .{ }^{1} \mathrm{H} \mathrm{NMR}\left(500 \mathrm{MHz}, \mathrm{CDCl}_{3}\right): \delta[\mathrm{ppm}]=0.18(\mathrm{~s}$, 9H), $1.07(\mathrm{~s}, 3 \mathrm{H}), 1.09(\mathrm{~s}, 18 \mathrm{H}), 5.82(\mathrm{~d}, J=11.3 \mathrm{~Hz}, 1 \mathrm{H}), 5.84(\mathrm{~d}, J=11.3 \mathrm{~Hz}, 1 \mathrm{H}) .{ }^{13} \mathrm{C} \mathrm{NMR}(125 \mathrm{MHz}$, $\left.\mathrm{CDCl}_{3}\right): \delta[\mathrm{ppm}]=-0.3,11.2,18.7,100.0,102.0,103.0,103.7,120.0,120.5$.

same as lit. ${ }^{[2]}$

(Z)-Hex-3-en-1,5-diynyl-triisopropyl-silane (18). $1.38 \mathrm{~g} 16$ (4.5 mmol) was dissolved in $70 \mathrm{~mL}$ of benzene/methanol (1:1). $0.69 \mathrm{~g}$ of $\mathrm{K}_{2} \mathrm{CO}_{3}$ (5.0 mmol, 1.1 equiv) was added and the reaction mixture was stirred for $2 \mathrm{~h}$ at $\mathrm{rt}$. Water $(50 \mathrm{~mL})$ was added and the aqueous phase was extracted with hexanes $(3 \times 50 \mathrm{~mL})$. The organic phases were washed with brine, dried over anhydrous $\mathrm{MgSO}_{4}$ and evaporated to leave a dark oil $(1.01 \mathrm{~g}, 4.3 \mathrm{mmol}, 96 \%) . \mathrm{R}_{f}(\mathrm{PE})=0.66 .{ }^{1} \mathrm{H} \mathrm{NMR}\left(500 \mathrm{MHz}, \mathrm{CDCl}_{3}\right)$ : $\delta[\mathrm{ppm}]=1.07(\mathrm{~s}, 3 \mathrm{H}), 1.08(\mathrm{~s}, 18 \mathrm{H}), 3.30(\mathrm{dd}, J=1.2,0.7 \mathrm{~Hz}, 1 \mathrm{H}), 5.81(\mathrm{dd}, J=11.0,1.2 \mathrm{~Hz}, 1 \mathrm{H}), 5.91(\mathrm{dd}$, $J=11.0,0.7 \mathrm{~Hz}, 1 \mathrm{H}) .{ }^{13} \mathrm{C} \mathrm{NMR}\left(125 \mathrm{MHz}, \mathrm{CDCl}_{3}\right): \delta[\mathrm{ppm}]=11.2,18.7,80.9,84.8,100.3,103.4,119.3$, 122.0 .

same as lit. ${ }^{[2]}$

(Z)-(6-Bromo-hexa-3-en-1,5-diynyl)-triisopropyl-silane (17). $1.22 \mathrm{~g}$ of $\mathbf{1 6}$ (4.0 mmol, 1.0 equiv) was dissolved in $40 \mathrm{~mL}$ of acetone at $0{ }^{\circ} \mathrm{C} .0 .80 \mathrm{~g}$ of NBS (4.4 mmol, 1.1 equiv) and $0.14 \mathrm{~g}$ of $\mathrm{AgNO}_{3}(0.8 \mathrm{mmol}, 0.2$ equiv) were added and the reaction mixture was stirred overnight while warming to $\mathrm{rt}$. Water $(50 \mathrm{~mL})$ was added and the aqueous phase was extracted with hexanes $(4 \mathrm{x} 50 \mathrm{~mL})$. The organic phases were washed with brine, dried over anhydrous $\mathrm{MgSO}_{4}$ and evaporated to leave a slightly brown oil (1.24 g, $4.0 \mathrm{mmol}, 99 \%$ ), which was used without purification. $\mathrm{R}_{f}$ (petroleum ether) $=0.65$. IR (neat):

$\left[\mathrm{cm}^{-1}\right]=2943,2890,2865,2189,2134,1674,1569,1462,1384,1366,1241,1078,996,919,883,772$, 743. ${ }^{1} \mathrm{H}$ NMR $\left(500 \mathrm{MHz}, \mathrm{CDCl}_{3}\right): \delta[\mathrm{ppm}]=1.08(\mathrm{~s}, 3 \mathrm{H}), 1.10(\mathrm{~s}, 18 \mathrm{H}), 5.78(\mathrm{~d}, J=10.8 \mathrm{~Hz}, 1 \mathrm{H}), 5.88(\mathrm{~d}$, $J=10.8 \mathrm{~Hz}, 1 \mathrm{H}) .{ }^{13} \mathrm{C} \mathrm{NMR}\left(125 \mathrm{MHz}, \mathrm{CDCl}_{3}\right): \delta[\mathrm{ppm}]=11.2,18.7,57.5,78.3,100.3,103.4,119.8,121.9$. HRMS (CI): calcd. for $\mathrm{C}_{15} \mathrm{H}_{23} \mathrm{BrSi}\left[\mathrm{M}^{+}\right]$: 310.0752; found: 310.0750 .

(Z, Z)-(6-Bromo-hexa-3,5-dien-1-ynyl)-triisopropyl-silane (13). $5.2 \mathrm{~mL}$ of $\mathrm{BH}_{3} \cdot \mathrm{THF}(1 \mathrm{M}$ in THF, $5.2 \mathrm{mmol}, 1.3$ equiv) was added to $1.05 \mathrm{~mL}$ of cyclohexene (10.4 mmol, 2.6 equiv) at $0{ }^{\circ} \mathrm{C}$ and the mixture was stirred for $1.5 \mathrm{~h}$, while a white suspension formed. The reaction mixture was diluted with $50 \mathrm{~mL}$ of pentane and $1.24 \mathrm{~g}$ of $\mathbf{1 7}(4.0 \mathrm{mmol})$ in $50 \mathrm{~mL}$ pentane was added. The suspension cleared within $5 \mathrm{~min}$. After $30 \mathrm{~min} 0.2 \mathrm{~mL}$ of $\mathrm{AcOH}$ (16 mmol, 4.0 equiv) was added, the mixture wasstirred for $15 \mathrm{~min}$, and $1.93 \mathrm{~mL}$ of ethanolamine ( $32 \mathrm{mmol}, 8.0$ equiv) was added. After additional stirring for $10 \mathrm{~min}$, the mixture was filtered through a silica pad with hexanes and the filtrate was evaporated to afford a dark oil $(\sim 1.1 \mathrm{~g})$, which contained the product of ca. $90 \%$ purity $\left({ }^{1} \mathrm{H}-\mathrm{NMR}\right)$. Chromatography (basic alumina, hexanes $100 \%$ ) yielded a slightly coloured oil $(0.71 \mathrm{~g}, 2.3 \mathrm{mmol}, 57 \%, 2$ steps $) . \mathrm{R}_{f}(\mathrm{PE})=0.66$. IR (neat):

$\left[\mathrm{cm}^{-1}\right]=3081,2943,2891,2865,2137,1627,1604,1549,1462,1383,1366,1296,1241,1079,1016$, 996, 980, 919, 883, 782, 735, 711. ${ }^{1} \mathrm{H}$ NMR (500 MHz, $\left.\mathrm{CDCl}_{3}\right): \delta[\mathrm{ppm}]=1.07(\mathrm{~s}, 18 \mathrm{H}), 1.09(\mathrm{~s}, 3 \mathrm{H}), 5.70$ (ddd, $J=10.9,1.4,1.0 \mathrm{~Hz}, 1 \mathrm{H}), 6.35$ (dt, $J=7.2,1.3 \mathrm{~Hz}, 1 \mathrm{H}), 6.72(\mathrm{td}, J=10.7,1.0 \mathrm{~Hz}, 1 \mathrm{H}), 7.20$ (ddd, $J=$ 10.6, 7.2, 1.0 Hz, 1H). ${ }^{13} \mathrm{C}$ NMR $\left(125 \mathrm{MHz} \mathrm{CDCl}_{3}\right): \delta[\mathrm{ppm}]=11.2,18.7,100.0,103.5,112.0,113.5,129.9$, 135.6. HRMS (CI): calcd. for $\mathrm{C}_{15} \mathrm{H}_{25} \mathrm{BrSi}\left[\mathrm{M}^{+}\right]$: 312.0909 ; found: 312.0890 . 


\section{(Z)-(1S,2S,4S)-1-(2-Phenyl-[1,3]dioxan-4-yl)-7-triisopropylsilanyl-hept-4-en-2,6-diyn-1-}

ol (19). $370 \mathrm{mg}$ of $\mathrm{Zn}(\mathrm{OTf})_{2}(1.0 \mathrm{mmol}, 1.25$ equiv) and $185 \mathrm{mg}$ of (+)-NME (1.0 mmol, 1.25 equiv) were suspended in $5 \mathrm{~mL}$ of toluene at rt. $390 \mu \mathrm{L}$ of $\mathrm{NEt}_{3}(3.0 \mathrm{mmol}, 3.8$ equiv) was added and the mixture was stirred for $2 \mathrm{~h} .280 \mathrm{mg}$ of $\mathbf{1 8}$ (1.2 mmol, 1.5 equiv) was added, the mixture was stirred for $15 \mathrm{~min}$ and $155 \mathrm{mg}$ of 10 ( $0.8 \mathrm{mmol}, 1.0$ equiv) was added. After stirring for $2 \mathrm{~h}$ at $\mathrm{rt}$, TLC showed complete conversion, and the mixture was extracted with saturated $\mathrm{NH}_{4} \mathrm{Cl} \cdot \mathrm{aq}$ and $\mathrm{Et}_{2} \mathrm{O}$. The organic phases were washed with brine, dried over $\mathrm{MgSO}_{4}$, filtered through a silica gel pad and evaporated. Purification via Chromatotron (hexanes:ethyl acetate 9:1 to 4:1) gave a clear oil $(260 \mathrm{mg}, 0.61 \mathrm{mmol}, 77 \%)$. $\mathrm{R}_{f}$ (hexanes:ethyl acetate 2:1) = 0.65. $[\alpha]^{20}{ }_{589}+43.0\left(\mathrm{c}=0.66, \mathrm{CHCl}_{3}\right)$. IR (neat): $\left[\mathrm{cm}^{-1}\right]=3038,2942,2890,2864,2138,1464,1394$, 1362, 1306, 1240, 1214, 1128, 1098, 1074, 1018, 918, 883, 848, 750. ${ }^{1} \mathrm{H}$ NMR (500 $\left.\mathrm{MHz}_{2} \mathrm{CDCl}_{3}\right)$ : $\delta[\mathrm{ppm}]=1.10(\mathrm{~s}, 21 \mathrm{H}), 1.77-1.83(\mathrm{~m}, 1 \mathrm{H}), 1.87(\mathrm{qd}, J=12.0,5.1 \mathrm{~Hz}, 1 \mathrm{H}), 2.60(\mathrm{brs}, J=6.1 \mathrm{~Hz}, 1 \mathrm{H}), 3.93-$ $3.98(\mathrm{~m}, 2 \mathrm{H}), 4.23(\mathrm{ddd}, J=11.5,5.0,1.3 \mathrm{~Hz}, 1 \mathrm{H}), 4.53(\mathrm{~d}, J=6.9 \mathrm{~Hz}, 1 \mathrm{H}), 5.52(\mathrm{~s}, 1 \mathrm{H}), 5.85(\mathrm{dd}, J=11.0$, $1.8 \mathrm{~Hz}, 1 \mathrm{H}), 5.89(\mathrm{~d}, J=11.0 \mathrm{~Hz}, 1 \mathrm{H}), 7.33-7.36(\mathrm{~m}, 3 \mathrm{H}), 7.46-7.49(\mathrm{~m}, 2 \mathrm{H}) .{ }^{13} \mathrm{C}$ NMR $\left(125 \mathrm{MHz}, \mathrm{CDCl}_{3}\right)$ : $\delta[\mathrm{ppm}]=11.2,18.6,27.2,66.3,66.5,79.6,83.9,93.2,100.1,101.3,103.5,119.1,120.8,126.1,128.3,129.0$, 138.0. HRMS (EI): calc. for $\mathrm{C}_{26} \mathrm{H}_{36} \mathrm{O}_{3} \mathrm{SiNa}\left[\mathrm{M}+\mathrm{Na}^{+}\right]$: 447.2331; found: 447.2342.

\section{(Z)-(1S,2S,4S)-Triisopropyl-[7-methoxymethoxy-7-(2-phenyl-[1,3]dioxan-4-yl)-hept-3-} en-1,5-diynyl]-silane (20). $1.70 \mathrm{~g}$ of 19 (4.0 mmol, 1.0 equiv) and $875 \mu \mathrm{L}$ of $i$ - $\operatorname{Pr}_{2} \mathrm{NEt}$ (5.0 mmol, 1.25 equiv) were dissolved in $60 \mathrm{~mL}$ of $\mathrm{CH}_{2} \mathrm{Cl}_{2}$ at $0{ }^{\circ} \mathrm{C}$ and $750 \mu \mathrm{L}$ of $\mathrm{MOMCl}(85 \%, 8.0 \mathrm{mmol}, 2.0$ equiv) were added dropwise. The mixture was stirred for $13 \mathrm{~h}$ at $\mathrm{rt}$ (TLC: low conversion), then a catalytic amount of DMAP $(\sim 30 \mathrm{mg}$ ) was added and the mixture was heated to reflux for $36 \mathrm{~h}$. The mixture was extracted with saturated $\mathrm{NH}_{4} \mathrm{Cl} \cdot \mathrm{aq}$. and $\mathrm{Et}_{2} \mathrm{O}$, the organic phases were washed with brine, dried over $\mathrm{MgSO}_{4}$ and evaporated. Purification via chromatography (silica gel, hexanes:ethyl acetate 9:1) afforded a clear oil (1.62 g, 3.46 mmol, 86\%). $\mathrm{R}_{f}$ (hexanes: ethyl acetate 4:1) =0.63. $[\alpha]_{589}^{20}+43.0\left(\mathrm{c}=0.69, \mathrm{CHCl}_{3}\right)$. IR (neat): $\left[\mathrm{cm}^{-}\right.$ $\left.{ }^{1}\right]=3038,2942,2890,2864,2142,1720,1572,1462,1390,1365,1298,1242,1215,1146,1129,1098$, $1027,918,882,751 .{ }^{1} \mathrm{H}$ NMR $\left(500 \mathrm{MHz}_{,} \mathrm{CDCl}_{3}\right): \delta[\mathrm{ppm}]=1.07(\mathrm{~s}, 3 \mathrm{H}), 1.10(\mathrm{~s}, 18 \mathrm{H}), 1.81(\mathrm{bd}, J=$ $13.2 \mathrm{~Hz}, 1 \mathrm{H}), 1.89(\mathrm{qd}, J=12.3,5.0 \mathrm{~Hz}, 1 \mathrm{H}), 3.37(\mathrm{~s}, 3 \mathrm{H}), 3.97(\mathrm{dd}, J=11.8,2.1 \mathrm{~Hz}, 1 \mathrm{H}), 4.06(\mathrm{dd}, J=8.6$, $2.2 \mathrm{~Hz}, 1 \mathrm{H}), 4.28(\mathrm{dd}, J=11.2,4.5 \mathrm{~Hz}, 1 \mathrm{H}), 4.61(\mathrm{~d}, J=5.8 \mathrm{~Hz}, 1 \mathrm{H}), 4.66(\mathrm{~d}, J=6.7 \mathrm{~Hz}, 1 \mathrm{H}), 4.93(\mathrm{~d}, J=$ $6.7 \mathrm{~Hz}, 1 \mathrm{H}), 5.52(\mathrm{~s}, 1 \mathrm{H}), 5.85(\mathrm{dd}, J=11.1,1.8 \mathrm{~Hz}, 1 \mathrm{H}), 5.89(\mathrm{~d}, J=11.1 \mathrm{~Hz}, 1 \mathrm{H}), 7.29-7.35(\mathrm{~m}, 3 \mathrm{H}), 7.46-$ $7.49(\mathrm{~m}, 2 \mathrm{H}) .{ }^{13} \mathrm{C} \mathrm{NMR}\left(125 \mathrm{MHz}, \mathrm{CDCl}_{3}\right): \delta[\mathrm{ppm}]=11.2,18.6,27.4,55.6,66.7,69.3,78.4,84.6,92.0$, $94.4,100.1,101.0,103.5,119.0,120.6,126.1,128.1,128.7,138.4$. HRMS (EI): calc. for $\mathrm{C}_{28} \mathrm{H}_{40} \mathrm{O}_{4} \mathrm{NaSi}$ $\left[\mathrm{M}+\mathrm{Na}^{+}\right]:$491.2594; found: 491.2598 .

\section{(Z,Z)-(1S,2S,4S)-Triisopropyl-[7-methoxymethoxy-7-(2-phenyl-[1,3]dioxan-4-yl)-hept-} 3,5-dien-1-ynyl]-silane (11). $622 \mathrm{mg}$ of 20 (1.32 mmol, 1.0 equiv), $470 \mu \mathrm{L}$ of quinoline (4.0 mmol, 1.3 equiv) and $440 \mathrm{mg}$ of Lindlar's catalyst ( $5 \mathrm{w} / \mathrm{w} \% \mathrm{Pd}$ on $\mathrm{CaCO}_{3} / \mathrm{Pb}, 70 \mathrm{w} / \mathrm{w} \%$ ) were suspended in $50 \mathrm{~mL}$ of hexanes at rt. The mixture was degassed and purged with $\mathrm{H}_{2}$ (4-5x) and then stirred at $\mathrm{rt}$ under $\mathrm{H}_{2}-$ pressure (balloon, $1 \mathrm{~atm}$ ) for $21 \mathrm{~h}$. The reaction mixture was filtered through Celite and evaporated. Purification via chromatography (silica gel, hexanes:ethyl acetate 40:1 to 10:1) yielded a clear oil (613 mg, 1.30 mmol, 98\%). $\mathrm{R}_{f}$ (hexanes:ethyl acetate 4:1) =0.57. $[\alpha]_{589}^{20}-38.4\left(\mathrm{c}=0.76, \mathrm{CHCl}_{3}\right)$. IR (neat): $\quad\left[\mathrm{cm}^{-}\right.$ $\left.{ }^{1}\right]=2942,2889,2864,2138,1764,1642,1631,1596,1462,1426,1366,1310,1241,1212,1141,1099$, 1028, 995, 916, 882, 847, 750. ${ }^{1} \mathrm{H}$ NMR $\left(500 \mathrm{MHz}, \mathrm{CDCl}_{3}\right): \delta[\mathrm{ppm}]=1.07(\mathrm{~s}, 3 \mathrm{H}), 1.10(\mathrm{~s}, 18 \mathrm{H}), 1.46(\mathrm{dd}$, $J=13.3,1.4 \mathrm{~Hz}, 1 \mathrm{H}), 1.84(\mathrm{qd}, J=12.3,5.0 \mathrm{~Hz}, 1 \mathrm{H}), 3.34(\mathrm{~s}, 3 \mathrm{H}), 3.91-3.98(\mathrm{~m}, 2 \mathrm{H}), 4.26(\mathrm{dd}, J=11.3$, $4.2 \mathrm{~Hz}, 1 \mathrm{H}), 4.61(\mathrm{~d}, J=6.7 \mathrm{~Hz}, 1 \mathrm{H}), 4.63(\mathrm{~d}, J=6.7 \mathrm{~Hz}, 1 \mathrm{H}), 4.61-4.64(\mathrm{~m}, 1 \mathrm{H}), 5.52(\mathrm{~s}, 1 \mathrm{H}), 5.52(\mathrm{t}, J=$ $10.3 \mathrm{~Hz}, 1 \mathrm{H}), 5.61(\mathrm{~d}, J=10.6 \mathrm{~Hz}, 1 \mathrm{H}), 6.80(\mathrm{t}, J=10.9 \mathrm{~Hz}, 1 \mathrm{H}), 6.87(\mathrm{t}, J=11.1 \mathrm{~Hz}, 1 \mathrm{H}), 7.29-7.35(\mathrm{~m}, 3 \mathrm{H})$, 
7.46-7.49 (m, 2H). ${ }^{13} \mathrm{C}$ NMR (125 MHz, $\left.\mathrm{CDCl}_{3}\right): \delta[\mathrm{ppm}]=11.3,18.7,27.3,55.4,66.8,73.1,78.9,94.0$, 99.3, 101.1, 103.3, 112.4, 126.1, 128.1, 128.6, 129.3, 130.7, 135.0, 138.6. HRMS (EI): calc. for $\mathrm{C}_{28} \mathrm{H}_{42} \mathrm{O}_{4} \mathrm{NaSi}$ $\left[\mathrm{M}+\mathrm{Na}^{+}\right]$: 491.2750; found: 493.2760 .

\section{$(Z, Z)-(3 S, 4 S)-1-B e n z y l o x y-4-m e t h o x y m e t h o x y-10$-triisopropylsilanyl-deca-5,7-dien-9-}

yn-3-ol (9). $2.5 \mathrm{~mL}$ of DIBALH (1M in $\mathrm{CH}_{2} \mathrm{Cl}_{2}, 2.5 \mathrm{mmol}, 5$ equiv) was added to $225 \mathrm{mg}$ of 11 (0.48 mmol, 1.0 equiv) in $10 \mathrm{~mL} \mathrm{CH}_{2} \mathrm{Cl}_{2}$ at $-10{ }^{\circ} \mathrm{C}$, and the mixture was stirred for $1.5 \mathrm{~h}$. Saturated Rochelle's salt solution was added and the mixture was extracted with ethyl acetate (4 x $50 \mathrm{~mL})$. The organic phases were washed with brine, dried over $\mathrm{MgSO}_{4}$ and evaporated. Purification via Chromatotron (hexanes:ethyl acetate 9:1 to 4:1) afforded a clear oil (155 mg, $0.33 \mathrm{mmol}, 69 \%$ ). $\mathrm{R}_{f}$ (hexanes:ethyl acetate $2: 1)=0.57 .[\alpha]^{20}{ }_{589}+4.6\left(\mathrm{c}=1.57, \mathrm{CHCl}_{3}\right)$. IR $($ neat $): \quad\left[\mathrm{cm}^{-1}\right]=3468,2942,2890,2864,2138,1462,1366$, $1260,1210,1152,1098,1028,962,918,883,806,749 .{ }^{1} \mathrm{H}$ NMR $\left(500 \mathrm{MHz}, \mathrm{CDCl}_{3}\right): \delta[\mathrm{ppm}]=1.10(\mathrm{~s}$, $21 \mathrm{H}), 1.68-1.73(\mathrm{~m}, 1 \mathrm{H}), 1.80-1.84(\mathrm{~m}, 1 \mathrm{H}), 2.89(\mathrm{~d}, J=2.6 \mathrm{~Hz}, 1 \mathrm{H}), 3.36(\mathrm{~s}, 3 \mathrm{H}), 3.63-3.67(\mathrm{~m}, 2 \mathrm{H}), 3.76-$ $3.80(\mathrm{~m}, 1 \mathrm{H}), 4.38(\mathrm{dd}, J=9.2,6.7 \mathrm{~Hz}, 1 \mathrm{H}), 4.50(\mathrm{~s}, 2 \mathrm{H}), 4.54(\mathrm{~d}, J=6.6 \mathrm{~Hz}, 1 \mathrm{H}), 4.65(\mathrm{~d}, J=6.6 \mathrm{~Hz}, 1 \mathrm{H})$, $5.47(\mathrm{t}, J=10.9 \mathrm{~Hz}, 1 \mathrm{H}), 5.57(\mathrm{~d}, J=10.8 \mathrm{~Hz}, 1 \mathrm{H}), 6.68(\mathrm{t}, J=11.2 \mathrm{~Hz}, 1 \mathrm{H}), 6.86(\mathrm{t}, J=11.4 \mathrm{~Hz}, 1 \mathrm{H}), 7.26-$ $7.28(\mathrm{~m}, 1 \mathrm{H}), 7.30-7.33(\mathrm{~m}, 4 \mathrm{H}) .{ }^{13} \mathrm{C}$ NMR $\left(125 \mathrm{MHz}, \mathrm{CDCl}_{3}\right): \delta[\mathrm{ppm}]=11.3,18.7,32.7,55.6,67.7,71.9$, $73.2,74.7,93.8,99.3,103.3,112.4,127.6,127.7,128.4,130.1,130.9,134.9,138.2$. HRMS (EI): calc. for $\mathrm{C}_{28} \mathrm{H}_{44} \mathrm{O}_{4} \mathrm{NaSi}\left[\mathrm{M}+\mathrm{Na}^{+}\right.$]: 495.2907; found: 495.2924 .

5-Benzyloxy-2,2-dimethyl-benzo[1,3]dioxin-4-one (23). $0.52 \mathrm{~g}$ of NaH (60\% in mineral oil, $13 \mathrm{mmol}, 1.3$ equiv) was added to $1.94 \mathrm{~g}$ of 5-hydroxy-2,2-dimethyl-benzo[1,3]dioxin-4-one (22 $)^{[3]}$ (10.0 mmol, 1.0 equiv) in $25 \mathrm{~mL}$ of DMF at $0{ }^{\circ} \mathrm{C}$ and the mixture was stirred for $0.5 \mathrm{~h}$. The dark red solution was then treated with $1.8 \mathrm{~mL}$ of benzyl bromide (15 mmol, 1.5 equiv) and then stirred at $\mathrm{rt}$ overnight. Saturated aqueous $\mathrm{NH}_{4} \mathrm{Cl}$ was added and the mixture was extracted with ethyl acetate $(3 \mathrm{x}$ $100 \mathrm{~mL}$ ). The organic phases were washed with brine, dried over $\mathrm{MgSO}_{4}$ and evaporated. The residue was dissolved in ethyl acetate and filtered through a silica gel pad. The filtrate was evaporated and recrystallized from $\mathrm{Et}_{2} \mathrm{O} / \mathrm{EtOAc}(1: 1)$ to yield fine, white crystals $(2.72 \mathrm{~g}, 9.6 \mathrm{mmol}, 96 \%) . \mathrm{R}_{f}$ (hexanes:ethyl acetate $\left.2: 1\right)$ $=0.41 . \mathrm{mp}\left(\mathrm{EtOAc} / \mathrm{Et}_{2} \mathrm{O}\right)=89-91{ }^{\circ} \mathrm{C}$. IR (neat): $\quad\left[\mathrm{cm}^{-1}\right]=2996,2942,1736,1607,1585,1498,1481$, $1453,1388,1378,1331,1259,1239,1204,1150,1093,1075,1038,963,921,849,803,763,737 .{ }^{1} \mathrm{H}$ NMR $\left(500 \mathrm{MHz}_{\mathrm{CDCl}}\right): \delta[\mathrm{ppm}]=1.69(\mathrm{~s}, 6 \mathrm{H}), 5.23(\mathrm{~s}, 2 \mathrm{H}), 6.53(\mathrm{~d}, J=8.2 \mathrm{~Hz}, 1 \mathrm{H}), 6.63(\mathrm{~d}, J=8.4 \mathrm{~Hz}, 1 \mathrm{H})$, $7.28(\mathrm{t}, J=8.3 \mathrm{~Hz}, 1 \mathrm{H}), 7.36(\mathrm{t}, J=7.8 \mathrm{~Hz}, 2 \mathrm{H}), 7.37(\mathrm{t}, J=8.2 \mathrm{~Hz}, 1 \mathrm{H}), 7.52(\mathrm{~d}, J=7.7 \mathrm{~Hz}, 2 \mathrm{H}) .{ }^{13} \mathrm{C} \mathrm{NMR}$ $\left(125 \mathrm{MHz}, \mathrm{CDCl}_{3}\right): \delta[\mathrm{ppm}]=25.6,70.6,103.8,105.2,107.1,109.4,126.6,127.8,128.6,136.2,136.2,157.8$, 158.0, 160.3. HRMS (CI): calcd. for $\mathrm{C}_{17} \mathrm{H}_{16} \mathrm{O}_{4}\left[\mathrm{M}^{+}\right]$: 284.1049; found: 284.1039 .

\section{(Z,Z)-(3S,4S)-2-Benzyloxy-6-hydroxy-benzoic acid 3-(1-benzyloxy-4-methoxymethoxy-deca-} 5,7-dien-9-yn)-yl ester (21). $378 \mathrm{mg}$ of $9(0.8 \mathrm{mmol})$ was azeotroped with benzene at $40{ }^{\circ} \mathrm{C}$, then dissolved in $30 \mathrm{~mL}$ of THF at $0{ }^{\circ} \mathrm{C}$ and treated with $0.96 \mathrm{~mL}$ of NaHMDS (1M in THF, $0.96 \mathrm{mmol}$, 1.2 equiv). The mixture was stirred for $1 \mathrm{~h}$ at $0{ }^{\circ} \mathrm{C} .342 \mathrm{mg}$ of $\mathbf{1 1}$ (1.2 mmol, 1.5 equiv) in $5 \mathrm{~mL}$ of THF were added and the mixture was stirred at $\mathrm{rt}$ for $4 \mathrm{~h}$. Saturated aqueous $\mathrm{NH}_{4} \mathrm{Cl}(10 \mathrm{~mL})$ was added and the mixture was extracted with EtOAc $(3 \times 50 \mathrm{~mL})$. The organic phases were dried over $\mathrm{MgSO}_{4}$ and evaporated. Purification via chromatography (Chromatotron, hexanes:EtOAc 6:1 to 4:1) afforded 21a as a colourless oil (433 mg, $0.62 \mathrm{mmol}, 77 \%) . \mathrm{R}_{f}$ (hexanes:ethyl acetate 4:1) $=0.54 .[\alpha]^{20}{ }_{589}-19.3$ (c =0.57, $\left.\mathrm{CHCl}_{3}\right)$. IR (neat):

$\left[\mathrm{cm}^{-1}\right]=3631,3372,3032,2942,2890,2864,2139,1732,1652,1608,1581,1497,1454,1366,1298$,

[3] Hadfield, A.; Schweitzer, H.; Trova, M. P.; Green, K. Synth. Commun. 1994, 1025. 
1256, 1228, 1174, 1154, 1072, 1030, 966, 918, 882, 811, 745. ${ }^{1} \mathrm{H}$ NMR $\left(500 \mathrm{MHz}, \mathrm{CDCl}_{3}\right): \delta[\mathrm{ppm}]=1.06$ $(\mathrm{s}, 3 \mathrm{H}), 1.09(\mathrm{~s}, 18 \mathrm{H}), 1.54-1.59(\mathrm{~m}, 1 \mathrm{H}), 1.99-2.04(\mathrm{~m}, 1 \mathrm{H}), 3.20(\mathrm{~s}, 3 \mathrm{H}), 3.31-3.38(\mathrm{~m}, 2 \mathrm{H}), 4.33(\mathrm{~d}, J=$ $11.9 \mathrm{~Hz}, 1 \mathrm{H}), 4.38(\mathrm{~d}, J=11.9 \mathrm{~Hz}, 1 \mathrm{H}), 4.43(\mathrm{~d}, J=6.8 \mathrm{~Hz}, 1 \mathrm{H}), 4.51(\mathrm{t}, J=7.0 \mathrm{~Hz}, 1 \mathrm{H}), 4.53(\mathrm{~d}, J=6.8 \mathrm{~Hz}$, $1 \mathrm{H}), 4.98(\mathrm{~d}, J=11.0 \mathrm{~Hz}, 1 \mathrm{H}), 4.99(\mathrm{~d}, J=11.0 \mathrm{~Hz}, 1 \mathrm{H}), 5.39(\mathrm{t}, J=9.0 \mathrm{~Hz}, 1 \mathrm{H}), 5.47-5.50(\mathrm{~m}, 1 \mathrm{H}), 5.54(\mathrm{~d}$, $J=10.8 \mathrm{~Hz}, 1 \mathrm{H}), 6.45(\mathrm{~d}, J=8.3 \mathrm{~Hz}, 1 \mathrm{H}), 6.50(\mathrm{t}, J=11.5 \mathrm{~Hz}, 1 \mathrm{H}), 6.60(\mathrm{~d}, J=8.4 \mathrm{~Hz}, 1 \mathrm{H}), 6.79(\mathrm{t}, J=$ $11.2 \mathrm{~Hz}, 1 \mathrm{H}), 7.18-7.35(\mathrm{~m}, 10 \mathrm{H}), 7.43(\mathrm{~d}, J=7.1 \mathrm{~Hz}, 1 \mathrm{H}), 11.49$ (s, 1H). ${ }^{13} \mathrm{C}$ NMR $\left(125 \mathrm{MHz}, \mathrm{CDCl}_{3}\right)$ : $\delta[\mathrm{ppm}]=11.3,18.6,30.4,55.4,66.0,71.0,72.1,73.1,73.3,93.8,99.4,103.2,103.6,110.3,112.4,127.5$, $127.7,128.0,128.0,128.0,128.3,128.4,129.2$, 131.0, 134.7, 135.0, 136.5, 138.2, 160.1, 163.7, 170.5. HRMS (EI): calcd. for $\mathrm{C}_{42} \mathrm{H}_{54} \mathrm{O}_{7} \mathrm{SiNa}\left[\mathrm{M}+\mathrm{Na}^{+}\right]$: 721.3537; found: 721.3545 .

$0.84 \mathrm{~mL}$ of TBAF (1M in THF, $0.84 \mathrm{mmol}, 1.5$ equiv) was added to $391 \mathrm{mg}$ of the crude addition product $(0.56 \mathrm{mmol})$ in $30 \mathrm{~mL}$ of THF at $0{ }^{\circ} \mathrm{C}$ and the mixture was stirred for $1 \mathrm{~h}$ at $0{ }^{\circ} \mathrm{C}$. The mixture was filtered through a silica gel pad and evaporated. Purification via chromatography (Chromatotron, hexanes:EtOAc $6: 1)$ yielded 21 as a colourless oil $(229 \mathrm{mg}, 0.42 \mathrm{mmol}, 75 \%)$. $\mathrm{R}_{f}$ (hexanes:ethyl acetate $\left.4: 1\right)=0.23 .[\alpha]^{20}{ }_{589}$ $-7.8\left(\mathrm{c}=1.21, \mathrm{CHCl}_{3}\right)$. IR (neat): $\quad\left[\mathrm{cm}^{-1}\right]=3285,3063,3032,2931,2888,1732,1656,1652,1607,1581$, 1496, 1455, 1373, 1298, 1257, 1228, 1176, 1153, 1090, 1072, 1029, 916, 845, 811, 746. ${ }^{1} \mathrm{H}$ NMR $\left(500 \mathrm{MHz}, \mathrm{CDCl}_{3}\right): \delta[\mathrm{ppm}]=1.60-1.60(\mathrm{~m}, 1 \mathrm{H}), 1.90-1.93(\mathrm{~m}, 1 \mathrm{H}), 3.21(\mathrm{~s}, 3 \mathrm{H}), 3.29(\mathrm{~d}, J=2.1 \mathrm{~Hz}, 1 \mathrm{H})$, $3.33-3.38(\mathrm{~m}, 2 \mathrm{H}), 4.36(\mathrm{~d}, J=11.9 \mathrm{~Hz}, 1 \mathrm{H}), 4.38(\mathrm{~d}, J=11.9 \mathrm{~Hz}, 1 \mathrm{H}), 4.44(\mathrm{~d}, J=6.8 \mathrm{~Hz}, 1 \mathrm{H}), 4.50-4.54(\mathrm{~m}$, $1 \mathrm{H}), 4.51(\mathrm{~d}, J=6.8 \mathrm{~Hz}, 1 \mathrm{H}), 4.98(\mathrm{~d}, J=11.0 \mathrm{~Hz}, 1 \mathrm{H}), 4.99(\mathrm{~d}, J=11.0 \mathrm{~Hz}, 1 \mathrm{H}), 5.43(\mathrm{t}, J=10.6 \mathrm{~Hz}, 1 \mathrm{H})$, $5.47(\mathrm{~d}, J=10.8 \mathrm{~Hz}, 1 \mathrm{H}), 5.47-5.50(\mathrm{~m}, 1 \mathrm{H}), 6.46(\mathrm{~d}, J=8.3 \mathrm{~Hz}, 1 \mathrm{H}), 6.59(\mathrm{t}, J=11.5 \mathrm{~Hz}, 1 \mathrm{H}), 6.61(\mathrm{~d}, J=$ $8.3 \mathrm{~Hz}, 1 \mathrm{H}), 6.76(\mathrm{t}, J=11.3 \mathrm{~Hz}, 1 \mathrm{H}), 7.18-7.35(\mathrm{~m}, 10 \mathrm{H}), 7.43(\mathrm{~d}, J=7.1 \mathrm{~Hz}, 1 \mathrm{H}), 11.48(\mathrm{~s}, 1 \mathrm{H}) .{ }^{13} \mathrm{C} \mathrm{NMR}$ $\left(125 \mathrm{MHz}_{\mathrm{CDCl}}\right): \delta[\mathrm{ppm}]=30.2,55.3,65.9,70.9,72.0,73.0,73.2,80.1,84.8,93.8,103.2,103.5,110.2$, $110.9,127.5,127.6,128.0,128.0,128.2$, 128.4, 129.7, 130.6, 135.0, 135.9, 136.4, 138.2, 160.0, 163.6, 170.4 . HRMS (EI): calc. for $\mathrm{C}_{33} \mathrm{H}_{34} \mathrm{O}_{7} \mathrm{Na}\left[\mathrm{M}+\mathrm{Na}^{+}\right]$: 565.2202; found: 565.2216.

(Z,Z)-(3S,4S)-2-Benzyloxy-6-trifluoromethanesulfonyloxy-benzoic acid 3-(1-benzyloxy4-methoxymethoxy-deca-5,7-dien-9-yn)-yl ester (24). $250 \mathrm{mg}$ of 21 (0.46 mmol) were dissolved in $5 \mathrm{~mL}$ of pyridine at $0{ }^{\circ} \mathrm{C}$ and $0.25 \mathrm{~mL}$ of $\mathrm{Tf}_{2} \mathrm{O}(1.5 \mathrm{mmol}, 3.0$ equiv $)$ was added. The mixture was stirred for $0.5 \mathrm{~h}$ at $0{ }^{\circ} \mathrm{C}$ and saturated $\mathrm{NH}_{4} \mathrm{Cl}$ solution $(10 \mathrm{~mL})$ was added. The mixture was extracted with EtOAc $(2 \mathrm{x}$ $50 \mathrm{~mL})$. The organic phases were washed with saturated $\mathrm{CuSO}_{4}$ solution $(2 \mathrm{x} 100 \mathrm{~mL})$ and brine, then dried over $\mathrm{MgSO}_{4}$ and evaporated. Purification via chromatography (Chromatotron, hexanes:EtOAc 9:1) afforded the product as a clear oil $(265 \mathrm{mg}, 0.39 \mathrm{mmol}, 85 \%) . \mathrm{R}_{f}$ (hexanes:ethyl acetate $\left.4: 1\right)=0.25 .[\alpha]^{20}{ }_{589}+18.7$ $\left(\mathrm{c}=0.90, \mathrm{CHCl}_{3}\right)$. IR (neat): $\quad\left[\mathrm{cm}^{-1}\right]=3291,3032,2916,1735,1612,1454,1425,1279,1216,1141,1072$, 1037, 952, 825, 751. ${ }^{1} \mathrm{H}$ NMR $\left(500 \mathrm{MHz}, \mathrm{CDCl}_{3}\right): \delta[\mathrm{ppm}]=1.93-1.99(\mathrm{~m}, 1 \mathrm{H}), 2.01-2.05(\mathrm{~m}, 1 \mathrm{H}), 3.26(\mathrm{~d}$, $J=2.1 \mathrm{~Hz}, 1 \mathrm{H}), 3.27(\mathrm{~s}, 3 \mathrm{H}), 3.44-3.48(\mathrm{~m}, 2 \mathrm{H}), 4.32(\mathrm{~d}, J=11.7 \mathrm{~Hz}, 1 \mathrm{H}), 4.41(\mathrm{~d}, J=11.7 \mathrm{~Hz}, 1 \mathrm{H}), 4.46(\mathrm{~d}$, $J=6.8 \mathrm{~Hz}, 1 \mathrm{H}), 4.52(\mathrm{~d}, J=6.8 \mathrm{~Hz}, 1 \mathrm{H}), 4.68(\mathrm{dd}, J=9.6,4.5 \mathrm{~Hz}, 1 \mathrm{H}), 5.08(\mathrm{~s}, 2 \mathrm{H}), 5.40-5.46(\mathrm{~m}, 2 \mathrm{H}), 5.50$ $(\mathrm{t}, J=9.3 \mathrm{~Hz}, 1 \mathrm{H}), 6.66$ (quin, $J=10.5 \mathrm{~Hz}, 2 \mathrm{H}), 6.97(\mathrm{~d}, J=6.1 \mathrm{~Hz}, 1 \mathrm{H}), 6.98(\mathrm{~d}, J=6.0 \mathrm{~Hz}, 1 \mathrm{H}), 7.22-7.25$ $(\mathrm{m}, 1 \mathrm{H}), 7.28-7.31(\mathrm{~m}, 5 \mathrm{H}), 7.33-7.38(\mathrm{~m}, 5 \mathrm{H}) .{ }^{13} \mathrm{C} \mathrm{NMR}\left(125 \mathrm{MHz}, \mathrm{CDCl}_{3}\right): \delta[\mathrm{ppm}]=31.0,55.5,66.4$, $71.1,71.3,73.0,75.0,80.2,84.5,93.7,110.7,112.3,113.2,118.1,118.5$ (q, $\left.{ }^{1} J_{\mathrm{C}, \mathrm{F}}=318 \mathrm{~Hz}\right), 127.4,127.7$, $127.7,128.3,128.5,128.7,129.7,130.3,131.6,135.3,136.0,138.4,146.9,157.1,162.8 .{ }^{19} \mathrm{~F} \mathrm{NMR}$ $\left(470 \mathrm{MHz}, \mathrm{CDCl}_{3}\right): \delta[\mathrm{ppm}]=-73.65$ (s). HRMS (EI): calcd. for $\mathrm{C}_{34} \mathrm{H}_{33} \mathrm{~F}_{3} \mathrm{O}_{9} \mathrm{SNa}\left[\mathrm{M}+\mathrm{Na}^{+}\right]: 697.1695$; found: 697.1663 .

\section{(4R,2'S,4'S)-Acetic acid 4-hydroxy-4-(2'-phenyl-[1,3]dioxan-4'-yl)-but-2-ynyl ester (33).}

$9.3 \mathrm{~g}$ of $\mathrm{Zn}(\mathrm{OTf})_{2}(25 \mathrm{mmol}, 2.5 \mathrm{equiv})$ and $4.6 \mathrm{~g}$ of $(+)-\mathrm{NME}$ (25 mmol, 2.5 equiv) were suspended in $50 \mathrm{~mL}$ of toluene at rt. $6.5 \mathrm{~mL}$ of $\mathrm{NEt}_{3}(50 \mathrm{mmol}, 5.0$ equiv) was added and the mixture was stirred for $2 \mathrm{~h}$. $2.5 \mathrm{~mL}$ of propargyl acetate (32) (25 mmol, 2.5 equiv) was added, the mixture was stirred for $15 \mathrm{~min}$ and 
$1.90 \mathrm{~g}$ of $\mathbf{1 0}^{[2]}$ (9.9 mmol) was added. After stirring for $1.5 \mathrm{~h}$ at $\mathrm{rt}$, TLC showed complete conversion, and the mixture was extracted with saturated $\mathrm{NH}_{4} \mathrm{Cl} \cdot \mathrm{aq}$ and $\mathrm{Et}_{2} \mathrm{O}$. The organic phases were washed with brine, dried over $\mathrm{MgSO}_{4}$, filtered through a silica gel pad and evaporated. Purification via chromatography (silica gel, hexanes:ethyl acetate 9:1 to 4:1) gave a clear oil $(2.57 \mathrm{~g}, 8.8 \mathrm{mmol}, 89 \%)$, which slowly solidified. $\mathrm{R}_{f}$ (hexanes:ethyl acetate 4:1) $=0.56 . \mathrm{mp}\left(\mathrm{CHCl}_{3}\right)=61-62{ }^{\circ} \mathrm{C} .[\alpha]^{20}{ }_{589}+12.0\left(\mathrm{c}=0.78, \mathrm{CHCl}_{3}\right)$. IR (neat):

$\left[\mathrm{cm}^{-1}\right]=3446,2929,2862,1742,1455,1433,1378,1224,1129,1105,1022,920,884,847,764,700$. ${ }^{1} \mathrm{H}$ NMR $\left(300 \mathrm{MHz}, \mathrm{CDCl}_{3}\right): \delta[\mathrm{ppm}]=1.71(\mathrm{dq}, J=13.3,1.5 \mathrm{~Hz}, 1 \mathrm{H}), 1.87(\mathrm{dq}, J=12.1,5.2 \mathrm{~Hz}, 1 \mathrm{H}), 2.07$ $(\mathrm{s}, 3 \mathrm{H}), 2.73(\mathrm{~d}, J=4.0 \mathrm{~Hz}, 1 \mathrm{H}), 3.91-4.03(\mathrm{~m}, 2 \mathrm{H}), 4.31$ (ddd, $J=11.5,6.5,1.1 \mathrm{~Hz}, 1 \mathrm{H}), 4.37-4.41(\mathrm{~m}, 1 \mathrm{H})$, $4.69(\mathrm{~s}, 1 \mathrm{H}), 4.70(\mathrm{~s}, 1 \mathrm{H}), 5.51(\mathrm{~s}, 1 \mathrm{H}), 7.33-7.38(\mathrm{~m}, 3 \mathrm{H}), 7.44-7.48(\mathrm{~m}, 2 \mathrm{H}) .{ }^{13} \mathrm{C} \mathrm{NMR}\left(75 \mathrm{MHz}, \mathrm{CDCl}_{3}\right)$ : $\delta[\mathrm{ppm}]=20.7(\mathrm{q}), 26.9(\mathrm{t}), 52.1(\mathrm{t}), 65.5(\mathrm{~d}), 66.4(\mathrm{t}), 79.3(\mathrm{~d}), 80.6(\mathrm{~s}), 83.2(\mathrm{~s}), 101.2(\mathrm{~d}), 126.1(\mathrm{~d}$ x2), $128.6(\mathrm{~d} \mathrm{x} 2), 129.1$ (d), 137.9 (s), 170.1 (s). HRMS (CI): calcd. for $\mathrm{C}_{16} \mathrm{H}_{18} \mathrm{O}_{5}$ [M $\mathrm{M}^{+}$: 290.1154; found: 290.1143.

(4R,2'S,4'S)-Acetic acid 4-methoxymethoxy-4-(2'-phenyl-[1,3]dioxan-4'-yl)-but-2-ynyl ester (34). $1.70 \mathrm{~g}$ of $33(5.9 \mathrm{mmol})$ in $15 \mathrm{~mL}$ of $\mathrm{CH}_{2} \mathrm{Cl}_{2}$ were treated with $1.1 \mathrm{~mL}$ of $\mathrm{MOMCl}(85 \%$, $12 \mathrm{mmol}, 2.0$ equiv) and $1.75 \mathrm{~mL}$ of $i$ - $\operatorname{Pr}_{2} \mathrm{NEt}\left(10 \mathrm{mmol}, 1.7\right.$ equiv) at $0{ }^{\circ} \mathrm{C}$ and stirred overnight. TLC indicated complete conversion and the mixture was extracted with saturated aq. $\mathrm{NH}_{4} \mathrm{Cl}$ and $\mathrm{CH}_{2} \mathrm{Cl}_{2}(2 \mathrm{x}$ $100 \mathrm{~mL}$ ). The organic phases were dried over anhydrous $\mathrm{MgSO}_{4}$ and evaporated. The resulting clear oil was used without further purification. $\mathrm{R}_{f}$ (hexanes:ethyl acetate 2:1) $=0.45$. $[\alpha]^{20}{ }_{589}+42.0\left(\mathrm{c}=1.46, \mathrm{CHCl}_{3}\right)$. IR (neat): $\quad\left[\mathrm{cm}^{-1}\right]=3035,2936,2889,1748,1636,1452,1435,1398,1377,1361,1314,1272,1223,1150$, 1129, 1101, 1069, 1026, 946, 918, 760, 715. ${ }^{1} \mathrm{H}$ NMR $\left(500 \mathrm{MHz}, \mathrm{CDCl}_{3}\right): \delta[\mathrm{ppm}]=1.73(\mathrm{~d}, J=13.3 \mathrm{~Hz}$, $1 \mathrm{H}), 1.94(\mathrm{dq}, J=12.4,5.0 \mathrm{~Hz}, 1 \mathrm{H}), 2.07(\mathrm{~s}, 3 \mathrm{H}), 3.36(\mathrm{~s}, 3 \mathrm{H}), 3.97(\mathrm{dq}, J=12.0,2.5 \mathrm{~Hz}, 1 \mathrm{H}), 4.03(\mathrm{ddd}, J=$ $11.5,7.0,2.5 \mathrm{~Hz}, 1 \mathrm{H}), 4.29(\mathrm{dd}, J=11.7,4.0 \mathrm{~Hz}, 1 \mathrm{H}), 4.49(\mathrm{dt}, J=7.0,1.6 \mathrm{~Hz}, 1 \mathrm{H}), 4.66(\mathrm{~d}, J=6.7 \mathrm{~Hz}, 1 \mathrm{H})$, $4.73(\mathrm{~d}, J=1.6 \mathrm{~Hz}, 2 \mathrm{H}), 4.89(\mathrm{~d}, J=6.7 \mathrm{~Hz}, 1 \mathrm{H}), 5.52(\mathrm{~s}, 1 \mathrm{H}), 7.30-7.33(\mathrm{~m}, 3 \mathrm{H}), 7.46-7.48(\mathrm{~m}, 2 \mathrm{H})$.

${ }^{13} \mathrm{C} \mathrm{NMR}\left(75 \mathrm{MHz}, \mathrm{CDCl}_{3}\right): \delta[\mathrm{ppm}]=20.7,27.2,52.1,55.7,66.6,68.6,78.1,81.5,82.0,94.5,101.1,126.1$, 128.1, 128.8, 138.3, 170.0. HRMS (EI): calc. for $\mathrm{C}_{18} \mathrm{H}_{22} \mathrm{O}_{6} \mathrm{Na}\left[\mathrm{M}+\mathrm{Na}^{+}\right]$: 357.1314; found: 357.1319 .

(4R,2'S,4'S)-4-Methoxymethoxy-4-(2'-phenyl-[1,3]dioxan-4'-yl)-but-2-yn-1-ol (35). $0.90 \mathrm{~g}$ of $\mathrm{K}_{2} \mathrm{CO}_{3}(6 \mathrm{mmol}, 1.0$ equiv) was added to $2.01 \mathrm{~g}$ of crude 34 ( $\sim 5.9 \mathrm{mmol})$ in $15 \mathrm{~mL}$ of $\mathrm{MeOH}$ at $0{ }^{\circ} \mathrm{C}$ and the mixture was stirred for $0.5 \mathrm{~h}$. The mixture was extracted with saturated aq. $\mathrm{NH}_{4} \mathrm{Cl}$ and EtOAc $(4 \times 50 \mathrm{~mL})$. The combined organic phases were washed with brine, dried over anhydrous $\mathrm{MgSO}_{4}$ and evaporated to yield a clear oil $(1.74 \mathrm{~g}, \sim 5.9 \mathrm{mmol} \sim 99 \%)$, which was used without further purification.

$\mathrm{R}_{f}$ (hexanes: ethyl acetate $\left.2: 1\right)=0.25 .[\alpha]^{20}{ }_{589}+50.8\left(\mathrm{c}=1.79, \mathrm{CHCl}_{3}\right)$. IR (neat): $\left[\mathrm{cm}^{-1}\right]=3395,2890$, 1714, 1669, 1456, 1399, 1366, 1266, 1243, 1218, 1146, 1124, 1102, 1067, 1026, 918, 880, 764, 732. ${ }^{1} \mathrm{H}$ NMR $\left(500 \mathrm{MHz}, \mathrm{CDCl}_{3}\right): \delta[\mathrm{ppm}]=1.74(\mathrm{~d}, J=13.2 \mathrm{~Hz}, 1 \mathrm{H}), 1.88(\mathrm{brt}, J=5.3 \mathrm{~Hz}, 1 \mathrm{H}), 1.97(\mathrm{dq}, J=$ $12.5,5.0 \mathrm{~Hz}, 1 \mathrm{H}), 3.37(\mathrm{~s}, 3 \mathrm{H}), 3.97(\mathrm{td}, J=10.7,2.4 \mathrm{~Hz}, 1 \mathrm{H}), 4.01-4.07(\mathrm{~m}, 1 \mathrm{H}), 4.26-4.34(\mathrm{~m}, 3 \mathrm{H}), 4.51(\mathrm{~d}$, $J=6.7 \mathrm{~Hz}, 1 \mathrm{H}), 4.66(\mathrm{~d}, J=6.7 \mathrm{~Hz}, 1 \mathrm{H}), 4.90(\mathrm{~d}, J=6.7 \mathrm{~Hz}, 1 \mathrm{H}), 5.52(\mathrm{~s}, 1 \mathrm{H}), 7.30-7.33(\mathrm{~m}, 3 \mathrm{H}), 7.46-7.48$ $(\mathrm{m}, 2 \mathrm{H}) .{ }^{13} \mathrm{C} \mathrm{NMR}\left(75 \mathrm{MHz}, \mathrm{CDCl}_{3}\right): \delta[\mathrm{ppm}]=27.2,51.0,55.7,66.6,68.6,78.2,80.9,85.8,94.4,101.1$, 126.1, 128.1, 128.8, 138.3. HRMS (EI): calc. for $\mathrm{C}_{16} \mathrm{H}_{20} \mathrm{O}_{5} \mathrm{Na}\left[\mathrm{M}+\mathrm{Na}^{+}\right]$: 315.1208; found: 315.1200 .

(Z)-(4R,2'S,4'S)-4-Methoxymethoxy-4-(2'-phenyl-[1,3]dioxan-4'-yl)-but-2-en-1-ol (36). $1.74 \mathrm{~g}$ of $25(5.9 \mathrm{mmol})$ and $0.12 \mathrm{~mL}$ of quinoline $(1.0 \mathrm{mmol}, 0.17$ equiv) in $30 \mathrm{~mL}$ of $\mathrm{MeOH}$ at $\mathrm{rt}$ were degassed and purged with $\mathrm{N}_{2}$ (repeated two times). $500 \mathrm{mg}$ of Lindlar's catalyst ( $5 \mathrm{w} / \mathrm{w} \% \mathrm{Pd}$ on $\mathrm{CaCO}_{3} / \mathrm{Pb}$, $30 \mathrm{w} / \mathrm{w} \%$ ) were added and the suspension was degassed and purged with $\mathrm{N}_{2}$ (three times), then with $\mathrm{H}_{2}$ (three times). The mixture was stirred under $\mathrm{H}_{2}$-atmosphere (balloon) for $24 \mathrm{~h}$. The mixture was extracted 
with aq. $15 \% \mathrm{KHSO}_{4}$ and $\mathrm{Et}_{2} \mathrm{O}(3 \times 100 \mathrm{~mL})$, the organic phases were washed with brine, dried over anhydrous $\mathrm{MgSO}_{4}$ and evaporated. The residue was subjected to chromatography (silica gel, hexanes:EtOAc 4:1) to yield a clear oil (1.42 g, $4.8 \mathrm{mmol}, 81 \%$ over 3 steps). $\mathrm{R}_{f}$ (hexanes:ethyl acetate $\left.1: 1\right)=0.29 .[\alpha]^{20}{ }_{589}$ $+37.9\left(\mathrm{c}=2.07, \mathrm{CHCl}_{3}\right)$. IR (neat): $\left[\mathrm{cm}^{-1}\right]=3430,2928,2863,1453,1399,1367,1244,1216,1140,1367$, 1244, 1028, 918, 759, 732. ${ }^{1} \mathrm{H}$ NMR $\left(500 \mathrm{MHz} \mathrm{CDCl}_{3}\right): \delta[\mathrm{ppm}]=1.51(\mathrm{brd}, J=11.9 \mathrm{~Hz}, 1 \mathrm{H}), 1.97(\mathrm{qd}$, $J=12.4,4.9 \mathrm{~Hz}, 1 \mathrm{H}), 2.11$ (brs, $1 \mathrm{H}), 3.37(\mathrm{~s}, 3 \mathrm{H}), 3.95(\mathrm{t}, J=8.0 \mathrm{~Hz}, 1 \mathrm{H}), 4.01-4.04(\mathrm{~m}, 1 \mathrm{H}), 4.07-4.10(\mathrm{~m}$, $1 \mathrm{H}), 4.18-4.22(\mathrm{~m}, 1 \mathrm{H}), 4.28(\mathrm{dd}, J=11.4,4.9 \mathrm{~Hz}, 1 \mathrm{H}), 4.59-6.64(\mathrm{~m}, 2 \mathrm{H}), 4.72(\mathrm{~d}, J=6.5 \mathrm{~Hz}, 1 \mathrm{H}), 5.49(\mathrm{t}$, $J=10.3 \mathrm{~Hz}, 1 \mathrm{H}), 5.53(\mathrm{~s}, 1 \mathrm{H}), 5.49(\mathrm{dt}, J=10.4,7.3 \mathrm{~Hz}, 1 \mathrm{H}), 7.30-7.36(\mathrm{~m}, 3 \mathrm{H}), 7.46-7.47(\mathrm{~m}, 2 \mathrm{H})$.

${ }^{13} \mathrm{C} \mathrm{NMR}\left(75 \mathrm{MHz}, \mathrm{CDCl}_{3}\right): \delta[\mathrm{ppm}]=26.5,55.4,58.3,66.8,72.8,78.6,94.1,101.2,126.0,127.9,128.2$, 128.8, 134.5, 138.3. HRMS (EI): calc. for $\mathrm{C}_{16} \mathrm{H}_{22} \mathrm{O}_{5} \mathrm{Na}\left[\mathrm{M}+\mathrm{Na}^{+}\right]$: 317.1365 ; found: 357.1364 .

\section{(Z)-(4R,2'S,4'S)-4-Methoxymethoxy-4-(2'-phenyl-[1,3]dioxan-4'-yl)-but-2-enal (31).} $855 \mathrm{mg}$ of36 (2.9 mmol) and $1.23 \mathrm{~g}$ of IBX (4.4 mmol, 1.5 equiv) in $15 \mathrm{~mL}$ of DMSO at rt were stirred for $1 \mathrm{~h}$ and then $\mathrm{H}_{2} \mathrm{O}$ and $\mathrm{Et}_{2} \mathrm{O}(100 \mathrm{~mL}$ each $)$ were added. The organic phase was extracted with $\mathrm{H}_{2} \mathrm{O}(5 \mathrm{x}$ $150 \mathrm{~mL}$ ), dried over anhydrous $\mathrm{MgSO}_{4}$ and evaporated to yield a clear oil, which was used immediately for the next reaction $(0.85 \mathrm{~g}, 2.8 \mathrm{mmol}, 97 \%) . \mathrm{R}_{f}$ (hexanes:ethyl acetate $\left.1: 1\right)=0.57 .{ }^{1} \mathrm{H} \mathrm{NMR}(500 \mathrm{MHz}$, $\left.\mathrm{CDCl}_{3}\right): \delta[\mathrm{ppm}]=1.52(\mathrm{~d}, J=13.0 \mathrm{~Hz}, 1 \mathrm{H}), 1.95(\mathrm{dq}, J=12.1,5.0 \mathrm{~Hz}, 1 \mathrm{H}), 3.35(\mathrm{~s}, 3 \mathrm{H}), 3.95(\mathrm{td}, J=11.9$, $2.0 \mathrm{~Hz}, 1 \mathrm{H}), 4.07-4.11(\mathrm{~m}, 1 \mathrm{H}), 4.28(\mathrm{dd}, J=11.3,4.8 \mathrm{~Hz}, 1 \mathrm{H}), 4.68(\mathrm{~s}, 2 \mathrm{H}), 5.03(\mathrm{dd}, J=8.6,5.5 \mathrm{~Hz}, 1 \mathrm{H})$, $5.52(\mathrm{~s}, 1 \mathrm{H}), 6.16(\mathrm{dd}, J=11.4,7.6 \mathrm{~Hz}, 1 \mathrm{H}), 6.52(\mathrm{dd}, J=11.4,9.0 \mathrm{~Hz}, 1 \mathrm{H}), 7.31-7.35(\mathrm{~m}, 3 \mathrm{H}), 7.40-7.42(\mathrm{~m}$, $2 \mathrm{H}), 10.09(\mathrm{~d}, J=7.5 \mathrm{~Hz}, 1 \mathrm{H}) .{ }^{13} \mathrm{C} \mathrm{NMR}\left(125 \mathrm{MHz}, \mathrm{CDCl}_{3}\right): \delta[\mathrm{ppm}]=26.5,55.7,66.6,73.2,78.2,95.1$, 101.3, 126.0, 128.1, 128.8, 132.9, 138.1, 146.0, 191.3.

\section{(Z)-(1S,2S,4S)-4-(5,5-Dibromo-1-methoxymethoxy-penta-2,4-dienyl)-2-phenyl-}

[1,3]dioxane (37). A solution of $3.85 \mathrm{~g}$ of $\mathrm{CBr}_{4}\left(11.6 \mathrm{mmol}, 4\right.$ equiv) in $80 \mathrm{~mL}$ of $\mathrm{CH}_{2} \mathrm{Cl}_{2}$ at $0{ }^{\circ} \mathrm{C}$ was treated with $6.08 \mathrm{~g}$ of $\mathrm{PPh}_{3}$ (23.2 mmol, 8 equiv). After $5 \mathrm{~min} 8.0 \mathrm{~mL}$ of $\mathrm{NEt}_{3}\left(61 \mathrm{mmol}^{2} 20\right.$ equiv) was added and after an additional $5 \mathrm{~min}, 0.85 \mathrm{~g}$ of crude $31(\sim 2.8 \mathrm{mmol})$ in $20 \mathrm{~mL}$ of $\mathrm{CH}_{2} \mathrm{Cl}_{2}$ was added. The reaction mixture was stirred for $0.75 \mathrm{~h}$ and then poured into $900 \mathrm{~mL}$ of hexanes. The whitish precipitate was filtered off through a silica gel pad and washed with $\mathrm{Et}_{2} \mathrm{O}(300 \mathrm{~mL})$. The filtrate was evaporated and the residue was purified by chromatography (Chromatotron, hexanes 100\% to hexanes:EtOAc 9:1) to yield a clear oil $\left(1.10 \mathrm{~g}, 2.45 \mathrm{mmol}, 84 \%\right.$ over 2 steps). $\mathrm{R}_{f}$ (hexanes:ethyl acetate $\left.6: 1\right)=0.78 .[\alpha]_{589}^{20}-7.7(\mathrm{c}=0.54$, $\mathrm{CHCl}_{3}$ ). IR (neat): $\quad\left[\mathrm{cm}^{-1}\right]=2856,1716,1558,1637,1600,1456,1374,1218,1172,1108,1068,1027$, $918,880,846,817,772 .{ }^{1} \mathrm{H}$ NMR $\left(500 \mathrm{MHz}, \mathrm{CDCl}_{3}\right): \delta[\mathrm{ppm}]=1.48(\mathrm{dq}, J=13.2,1.4 \mathrm{~Hz}, 1 \mathrm{H}), 1.86(\mathrm{dq}$, $J=12.0,4.8 \mathrm{~Hz}, 1 \mathrm{H}), 3.34(\mathrm{~s}, 3 \mathrm{H}), 3.94-4.01(\mathrm{~m}, 2 \mathrm{H}), 4.28(\mathrm{dd}, J=11.3,4.8 \mathrm{~Hz}, 1 \mathrm{H}), 4.53(\mathrm{dd}, J=9.2$, $6.3 \mathrm{~Hz}, 1 \mathrm{H}), 4.64(\mathrm{~s}, 2 \mathrm{H}), 5.52(\mathrm{~s}, 1 \mathrm{H}), 5.58(\mathrm{~m}, 1 \mathrm{H}), 6.17(\mathrm{td}, J=11.0,1.0 \mathrm{~Hz}, 1 \mathrm{H}), 7.32-7.36(\mathrm{~m}, 4 \mathrm{H}), 7.46-$ $7.48(\mathrm{~m}, 2 \mathrm{H}) .{ }^{13} \mathrm{C} \mathrm{NMR}\left(125 \mathrm{MHz}, \mathrm{CDCl}_{3}\right): \delta[\mathrm{ppm}]=26.9,55.5,66.7,73.9,78.6,94.4,95.0,101.1,126.0$, 128.1, 128.7, 129.8, 130.4, 132.3, 138.4. HRMS (EI): calc. for $\mathrm{C}_{17} \mathrm{H}_{20} \mathrm{Br}_{2} \mathrm{O}_{4} \mathrm{Na}\left[\mathrm{M}+\mathrm{Na}^{+}\right]$: 468.9626; found: 468.9649.

\section{(Z,Z)-(1S,2S,4S)-4-(5-Bromo-1-methoxymethoxy-penta-2,4-dienyl)-2-phenyl-}

[1,3]dioxane (38). $1.10 \mathrm{~g}$ of $37(2.45 \mathrm{mmol})$, and $57 \mathrm{mg}$ of $\mathrm{Pd}\left(\mathrm{PPh}_{3}\right)_{4}(0.049 \mathrm{mmol}, 2 \mathrm{~mol} \%)$ in $35 \mathrm{~mL}$ of benzene were degassed and purged with $\mathrm{Ar} .690 \mu \mathrm{L}$ of $n-\mathrm{Bu}_{3} \mathrm{SnH}$ (2.48 mmol, 1.01 equiv) were added and the mixture was stirred at rt overnight. The solvent was evaporated and the reaction mixture was purified by chromatography (Chromatotron, hexanes 100\% to hexanes:EtOAc 6:1) to yield a clear oil that crystallized on standing $(800 \mathrm{mg}, 2.15 \mathrm{mmol}, 88 \%)$. $\mathrm{R}_{f}$ (hexanes: ethyl acetate $\left.4: 1\right)=0.56 .[\alpha]_{589}^{20}-30.0(\mathrm{c}=1.63$, $\left.\mathrm{CHCl}_{3}\right) \cdot \operatorname{mp}\left(\mathrm{CHCl}_{3}\right)=75-76^{\circ} \mathrm{C}$. IR (neat): $\quad\left[\mathrm{cm}^{-1}\right]=2929,2848,1365,1292,1218,1139,1098,1027$, 771. ${ }^{1} \mathrm{H}$ NMR $\left(500 \mathrm{MHz}, \mathrm{CDCl}_{3}\right): \delta[\mathrm{ppm}]=1.45(\mathrm{~d}, J=12.8 \mathrm{~Hz}, 1 \mathrm{H}), 1.84(\mathrm{dq}, J=12.4,4.7 \mathrm{~Hz}, 1 \mathrm{H}), 3.34$ 
(s, 3H), 3.91-3.98 (m, 2H), 4.26 (dd, $J=11.2,4.6 \mathrm{~Hz}, 1 \mathrm{H}), 4.57(\mathrm{dd}, J=9.4,6.9 \mathrm{~Hz}, 1 \mathrm{H}), 4.59(\mathrm{~d}, J=6.7 \mathrm{~Hz}$, $1 \mathrm{H}), 4.62(\mathrm{~d}, J=6.7 \mathrm{~Hz}, 1 \mathrm{H}), 5.52(\mathrm{~s}, 1 \mathrm{H}), 5.58(\mathrm{t}, J=10.3 \mathrm{~Hz}, 1 \mathrm{H}), 6.32(\mathrm{~d}, J=7.3 \mathrm{~Hz}, 1 \mathrm{H}), 6.63(\mathrm{t}, J=$ $10.9 \mathrm{~Hz}, 1 \mathrm{H}), 7.01(\mathrm{dd}, J=10.6,7.4 \mathrm{~Hz}, 1 \mathrm{H}), 7.30-7.34(\mathrm{~m}, 3 \mathrm{H}), 7.46-7.48(\mathrm{~m}, 2 \mathrm{H}) .{ }^{13} \mathrm{C} \mathrm{NMR}(125 \mathrm{MHz}$, $\left.\mathrm{CDCl}_{3}\right): \delta[\mathrm{ppm}]=27.1,55.4,66.7,73.5,78.7,94.0,101.1,111.9,126.1,127.5,128.1,128.7,129.1,130.1$, 138.5. HRMS (EI): calc. for $\mathrm{C}_{17} \mathrm{H}_{21} \mathrm{BrO}_{4} \mathrm{Na}\left[\mathrm{M}+\mathrm{Na}^{+}\right]$: 391.0521 ; found: 391.0530 .

(Z,Z)-(3S,4S)-1-Benzyloxy-8-bromo-4-methoxymethoxy-octa-5,7-dien-3-ol (29). $0.800 \mathrm{~g}$ of $38(2.15 \mathrm{mmol})$ in $25 \mathrm{~mL}$ of $\mathrm{CH}_{2} \mathrm{Cl}_{2}$ was treated with $10.8 \mathrm{~mL}$ of DIBALH (1M in toluene, $10.8 \mathrm{mmol}$, 5.0 equiv) at $0{ }^{\circ} \mathrm{C}$ and stirred for $10 \mathrm{~min}$. The mixture was quenched with EtOAc and EtOH and the mixture was filtered through a silica gel pad. The solvent was evaporated and the residue was purified by chromatography (Chromatotron, hexanes:EtOAc 4:1 to 2:1) to yield a clear oil (601 mg, $1.61 \mathrm{mmol}, 75 \%)$. $\mathrm{R}_{f}$ (hexanes: ethyl acetate 2:1) $=0.21 .[\alpha]^{20}{ }_{589}+31.7\left(\mathrm{c}=1.51, \mathrm{CHCl}_{3}\right)$. IR (neat): $\left[\mathrm{cm}^{-1}\right]=3461,3084$, 3029, 2928, 2888, 1718, 1637, 1601, 1570, 1496, 1453, 1406, 1364, 1276, 1207, 1151, 1098, 1028, 919, 811, 737, 714. ${ }^{1} \mathrm{H}$ NMR $\left(500 \mathrm{MHz}, \mathrm{CDCl}_{3}\right): \delta[\mathrm{ppm}]=1.68-1.72(\mathrm{~m}, 1 \mathrm{H}), 1.79-1.82(\mathrm{~m}, 1 \mathrm{H}), 2.91(\mathrm{~d}, J=$ $3.1 \mathrm{~Hz}, 1 \mathrm{H}), 3.36(\mathrm{~s}, 3 \mathrm{H}), 3.63-3.68(\mathrm{~m}, 2 \mathrm{H}), 3.77-3.82(\mathrm{~m}, 1 \mathrm{H}), 4.33(\mathrm{dd}, J=9.6,6.8 \mathrm{~Hz}, 1 \mathrm{H}), 4.57(\mathrm{dd}, J=$ 9.4, $6.9 \mathrm{~Hz}, 1 \mathrm{H}), 4.49(\mathrm{~s}, 2 \mathrm{H}), 4.63(\mathrm{~d}, J=6.7 \mathrm{~Hz}, 1 \mathrm{H}), 5.53(\mathrm{t}, J=10.0 \mathrm{~Hz}, 1 \mathrm{H}), 6.26(\mathrm{dt}, J=7.2,1.3 \mathrm{~Hz}, 1 \mathrm{H})$, $6.60(\mathrm{t}, J=10.9 \mathrm{~Hz}, 1 \mathrm{H}), 6.92(\mathrm{dd}, J=10.7,7.2 \mathrm{~Hz}, 1 \mathrm{H}), 7.26-7.32(\mathrm{~m}, 5 \mathrm{H}) .{ }^{13} \mathrm{C}$ NMR $\left(125 \mathrm{MHz}, \mathrm{CDCl}_{3}\right)$ : $\delta[\mathrm{ppm}]=32.5,55.6,67.7,71.9,73.3,75.0,93.7,111.9,127.3,127.7,127.7,128.4,129.3,130.9,138.1$. HRMS (EI): calc. for $\mathrm{C}_{17} \mathrm{H}_{23} \mathrm{BrO}_{4} \mathrm{Na}\left[\mathrm{M}+\mathrm{Na}^{+}\right]$: 393.0677 ; found: 393.0689 .

2,2-Dimethyl-5-trimethylsilanylethynyl-benzo[1,3]dioxin-4-one (39). $1.63 \mathrm{~g}$ of $\mathbf{1 0}^{[3]}$ (5.0 mmol), $1.41 \mathrm{~mL}$ of trimethylsilylacetylene (10.0 mmol, 2.0 equiv), $40 \mathrm{mg}$ of CuI (0.2 mmol, $4 \mathrm{~mol} \%$ ), $231 \mathrm{mg}$ of $\mathrm{Pd}\left(\mathrm{PPh}_{3}\right)_{4}(0.2 \mathrm{mmol}, 4 \mathrm{~mol} \%)$ and $1.75 \mathrm{~mL}$ of $i-\operatorname{Pr}_{2} \mathrm{NEt}(10.0 \mathrm{mmol}, 2.0$ equiv) in $50 \mathrm{~mL}$ of $\mathrm{CH}_{2} \mathrm{Cl}_{2}$ were degassed and purged with $\mathrm{N}_{2}$ and the mixture was stirred for $24 \mathrm{~h}$. The reaction mixture was extracted with saturated aq. $\mathrm{NH}_{4} \mathrm{Cl} / \mathrm{Et}_{2} \mathrm{O}(3 \times 50 \mathrm{~mL})$, the organic phases were washed with brine, dried over $\mathrm{MgSO}_{4}$ and evaporated. The residue was purified by chromatography (Chromatotron, hexanes:EtOAc 19:1) to yield a clear oil (1.35 g, $4.9 \mathrm{mmol}, 98 \%)$, which solidified upon standing. $\mathrm{R}_{f}$ (hexanes:ethyl acetate 4:1) = 0.61. $\mathrm{mp}$ (hexanes $/$ EtOAc) $=60-61^{\circ} \mathrm{C}$. IR (neat): $\quad\left[\mathrm{cm}^{-1}\right]=2994,2959,2900,2155,1748,1670,1592$, $1578,1472,1438,1389,1378,1319,1286,1272,1250,1228,1207,1073,1045,967,924,846,808,775$, 760, 732. ${ }^{1} \mathrm{H}$ NMR $\left(500 \mathrm{MHz}, \mathrm{CDCl}_{3}\right): \delta[\mathrm{ppm}]=0.27(\mathrm{~s}, 9 \mathrm{H}), 1.69(\mathrm{~s}, 6 \mathrm{H}), 6.88(\mathrm{~d}, J=8.2 \mathrm{~Hz}, 1 \mathrm{H}), 7.25$ $(\mathrm{d}, J=8.0 \mathrm{~Hz}, 1 \mathrm{H}), 7.40(\mathrm{t}, J=8.0 \mathrm{~Hz}, 1 \mathrm{H}) .{ }^{13} \mathrm{C} \mathrm{NMR}\left(125 \mathrm{MHz}, \mathrm{CDCl}_{3}\right): \delta[\mathrm{ppm}]=-0.3,25.7,102.3$, 102.5, 105.6, 114.4, 117.5, 125.1, 129.2, 134.7, 156.5, 158.6. HRMS (CI): calcd. for $\mathrm{C}_{15} \mathrm{H}_{18} \mathrm{O}_{3} \mathrm{Si}$ $\left[\mathrm{M}^{+}\right]: 274.1025$; found: 274.1034 .

5-Ethynyl-2,2-dimethyl-benzo[1,3]dioxin-4-one (40). $206 \mathrm{mg}$ of $39(0.75 \mathrm{mmol})$ in $5 \mathrm{~mL}$ of THF is treated with $1.0 \mathrm{~mL}$ of TBAF (1M in THF, $1.0 \mathrm{mmol}, 1.3$ equiv) at $0{ }^{\circ} \mathrm{C}$. The mixture is stirred for $15 \mathrm{~min}$, then diluted with $50 \mathrm{~mL}$ of hexanes and filtered through a silica gel pad. The filtrate is evaporated to provide the product as an off-white solid $(150 \mathrm{mg}, 0.74 \mathrm{mmol}, 99 \%)$, which decomposes slowly upon standing. $\quad \mathrm{R}_{f}$ (hexanes:ethyl acetate 6:1) $=0.41 . \mathrm{mp}$ (hexanes $/$ EtOAc) $=81-82{ }^{\circ} \mathrm{C}$. IR (neat): $\left[\mathrm{cm}^{-1}\right]=$ $3255,2999,2104,1742,1593,1579,1474,1438,1390,1379,1318,1284,1253,1206,1168,1145,1075$, 1045, 1016, 964, 920, 848, 808, 773. ${ }^{1} \mathrm{H}$ NMR (300 MHz, $\left.\mathrm{CDCl}_{3}\right): \delta[\mathrm{ppm}]=1.69(\mathrm{~s}, 6 \mathrm{H}), 3.49(\mathrm{~s}, 1 \mathrm{H})$, $6.93(\mathrm{dd}, J=8.3,1.0 \mathrm{~Hz}, 1 \mathrm{H}), 7.28(\mathrm{dd}, J=7.7,1.0 \mathrm{~Hz}, 1 \mathrm{H}), 7.44(\mathrm{t}, J=7.8 \mathrm{~Hz}, 1 \mathrm{H}) .{ }^{13} \mathrm{C} \mathrm{NMR}(125 \mathrm{MHz}$, $\left.\mathrm{CDCl}_{3}\right): \delta[\mathrm{ppm}]=25.6,81.1,84.1,105.3,114.5,118.0,124.0,129.6,135.0,156.5,158.7$. HRMS (CI): calcd. for $\mathrm{C}_{12} \mathrm{H}_{10} \mathrm{O}_{3}\left[\mathrm{M}^{+}\right]$: 202.0630; found: 202.0637. 
Potassium (E)-2-(2,2-dimethyl-4-oxo-4H-benzo[1,3]dioxin-5-yl) vinyltrifluoroborate (41). $9.3 \mathrm{~mL}$ of 2,5-dimethylhexa-2,4-diene (65 mmol, 6 equiv) in $80 \mathrm{~mL}$ of THF was treated with $30 \mathrm{~mL}$ of $\mathrm{BH}_{3} \cdot \mathrm{THF}$ ( $1 \mathrm{M}$ in THF, $30 \mathrm{mmol}, 2.5$ equiv) at $0{ }^{\circ} \mathrm{C}$. The mixture was stirred for $2 \mathrm{~h}$, then $2.40 \mathrm{~g}$ of 40 (11.9 mmol) in $20 \mathrm{~mL}$ of THF was added and the mixture was stirred for $1 \mathrm{~h} .4 \mathrm{~mL}$ of $\mathrm{H}_{2} \mathrm{O}$ was added carefully and after 90 min $9 \mathrm{~mL}$ of $\mathrm{CH}_{2} \mathrm{O}\left(37 \%\right.$ in $\left.\mathrm{H}_{2} \mathrm{O}\right)$ was added. The mixture was stirred overnight, extracted with brine/EtOAc $(3 \times 150 \mathrm{~mL})$ and the organic phases were evaporated. The residue was dissolved in $40 \mathrm{~mL}$ of acetone and $9.4 \mathrm{~g}$ of $\mathrm{KHF}_{2}\left(120 \mathrm{mmol}, 10\right.$ equiv) in $15 \mathrm{~mL}$ of $\mathrm{H}_{2} \mathrm{O}$ was added. The mixture was stirred overnight, evaporated and the residue was extracted with hot acetone $(3 \times 30 \mathrm{~mL})$ and $\mathrm{MeCN}(50 \mathrm{~mL})$. The organic phases were evaporated and the residue was dissolved in acetone. After addition of $\mathrm{Et}_{2} \mathrm{O}$, a precipitate formed which was filtered off and dried. The product was obtained in two charges as a white or off-white solid $(2.47 \mathrm{~g}, 8.0 \mathrm{mmol}, 66 \%)$. $\mathrm{mp}\left(\right.$ acetone $\left./ \mathrm{Et}_{2} \mathrm{O}\right)=207-209{ }^{\circ} \mathrm{C}(\mathrm{dec}$.). ${ }^{1} \mathrm{H}$ NMR $\left(500 \mathrm{MHz}\right.$, acetone- $\left.d_{6}\right): \delta[\mathrm{ppm}]=1.71(\mathrm{~s}, 6 \mathrm{H}), 6.37\left(\mathrm{dq}, J_{\mathrm{H}-\mathrm{H}}=18.2 \mathrm{~Hz}, J_{\mathrm{H}-\mathrm{F}}=3.8 \mathrm{~Hz}, 1 \mathrm{H}\right), 6.86$ $(\mathrm{d}, J=7.7 \mathrm{~Hz}, 1 \mathrm{H}), 7.36(\mathrm{~d}, J=7.8 \mathrm{~Hz}, 1 \mathrm{H}), 7.52(\mathrm{t}, J=8.0 \mathrm{~Hz}, 1 \mathrm{H}), 7.61(\mathrm{~d}, J=18.2 \mathrm{~Hz}, 1 \mathrm{H}) .{ }^{13} \mathrm{C} \mathrm{NMR}$ $\left(125 \mathrm{MHz}\right.$, acetone- $\left.d_{6}\right): \delta[\mathrm{ppm}]=25.2,105.2,110.9,114.9,121.6,132.7\left(\mathrm{q}, J_{\mathrm{C}-\mathrm{B}}=4.4 \mathrm{~Hz}\right), 135.5,142.3$, 146.4, 156.9, 160.4. ${ }^{11} \mathrm{~B}$ NMR $\left(300 \mathrm{MHz}\right.$, acetone- $\left.d_{6}\right): \delta[\mathrm{ppm}]=3.6$ (brs). ${ }^{19} \mathrm{~F}$ NMR $(470 \mathrm{MHz}$, acetone$\left.d_{6}\right): \delta[\mathrm{ppm}]=-142.0(\mathrm{~s})$.

$(1 E, 3 Z, 5 Z)-(7 S, 8 S)-5-(10-B e n z y l o x y-8-h y d r o x y-7-m e t h o x y m e t h o x y-d e c a-1,3,5$-trienyl)2,2-dimethyl-benzo[1,3]dioxin-4-one (42). $112 \mathrm{mg}$ of $29(0.30 \mathrm{mmol}), 124 \mathrm{mg}$ of $41(0.4 \mathrm{mmol}$, 1.3 equiv), $390 \mathrm{mg}$ of $\mathrm{Cs}_{2} \mathrm{CO}_{3}\left(1.2 \mathrm{mmol}, 4\right.$ equiv) and $35 \mathrm{mg}$ of $\mathrm{Pd}\left(\mathrm{PPh}_{3}\right)_{4}(0.03 \mathrm{mmol}, 10 \mathrm{~mol} \%)$ in $11 \mathrm{~mL}$ of $\mathrm{THF} / \mathrm{H}_{2} \mathrm{O}(10: 1)$ was degassed and purged with $\mathrm{N}_{2}$ (three times). The mixture was heated to reflux for $4 \mathrm{~h}$, then cooled and extracted with saturated aq. $\mathrm{NH}_{4} \mathrm{Cl} / \mathrm{EtOAc}(3 \times 50 \mathrm{~mL})$. The organic phases were dried over $\mathrm{MgSO}_{4}$ and evaporated. The residue was purified by chromatography (Chromatotron, hexanes:EtOAc 4:1 to $2: 1)$ to yield a clear oil $(138 \mathrm{mg}, 0.28 \mathrm{mmol}, 93 \%) . \mathrm{R}_{f}$ (hexanes:ethyl acetate $\left.2: 1\right)=0.15$. $[\alpha]^{20}{ }_{589}+6.9$ $\left(\mathrm{c}=0.51, \mathrm{CHCl}_{3}\right)$. IR (neat): $\quad\left[\mathrm{cm}^{-1}\right]=2923,2889,1730,1674,1578,1555,1473,1456,1390,1379,1319$, $1270,1210,1147,1098,1080,1042,966,922,770,732 .{ }^{1} \mathrm{H}$ NMR $\left(500 \mathrm{MHz}, \mathrm{CDCl}_{3}\right): \delta[\mathrm{ppm}]=1.69(\mathrm{~s}$, $6 \mathrm{H}), 1.72-1.75(\mathrm{~m}, 1 \mathrm{H}), 1.80-1.84(\mathrm{~m}, 1 \mathrm{H}), 2.92(\mathrm{~d}, J=1.9 \mathrm{~Hz}, 1 \mathrm{H}), 3.37(\mathrm{~s}, 3 \mathrm{H}), 3.61-3.69(\mathrm{~m}, 2 \mathrm{H}), 3.78-$ $3.82(\mathrm{~m}, 1 \mathrm{H}), 4.45(\mathrm{dd}, J=9.7,6.9 \mathrm{~Hz}, 1 \mathrm{H}), 4.50(\mathrm{~s}, 2 \mathrm{H}), 4.56(\mathrm{~d}, J=6.6 \mathrm{~Hz}, 1 \mathrm{H}), 4.67(\mathrm{~d}, J=6.6 \mathrm{~Hz}, 1 \mathrm{H})$, $5.42(\mathrm{t}, J=10.4 \mathrm{~Hz}, 1 \mathrm{H}), 6.34(\mathrm{t}, J=11.0 \mathrm{~Hz}, 1 \mathrm{H}), 6.38(\mathrm{t}, J=11.0 \mathrm{~Hz}, 1 \mathrm{H}), 6.82-6.85(\mathrm{~m}, 2 \mathrm{H}), 7.22(\mathrm{dd}, J=$ $15.4,10.9 \mathrm{~Hz}, 1 \mathrm{H}), 7.23-7.26(\mathrm{~m}, 1 \mathrm{H}), 7.30-7.34(\mathrm{~m}, 5 \mathrm{H}), 7.43(\mathrm{t}, J=8.0 \mathrm{~Hz}, 1 \mathrm{H}), 7.74(\mathrm{~d}, J=15.4 \mathrm{~Hz}, 1 \mathrm{H})$.

${ }^{13} \mathrm{C}$ NMR $\left(125 \mathrm{MHz}, \mathrm{CDCl}_{3}\right): \delta[\mathrm{ppm}]=26.0,32.7,55.6,67.7,72.0,73.2,74.6,93.9,105.2,110.8,116.4$, 121.0, 125.3, 127.5, 127.6, 127.7, 128.4, 128.7, 132.2, 132.3, 135.1, 138.3, 141.5, 157.0, 160.3. HRMS (EI): calc. for $\mathrm{C}_{29} \mathrm{H}_{34} \mathrm{O}_{7} \mathrm{Na}\left[\mathrm{M}+\mathrm{Na}^{+}\right]$: 517.2202; found: 517.2182 .

(9Z,12E)-(7S,8S)-7-(2-Benzyloxyethyl)-4-hydroxy-8-methoxymethoxy-11-methylene8,11-dihydro-7H-6-oxa-benzocyclodecen-5-one (44). $46.7 \mathrm{mg}$ of $\mathbf{2 9}(0.126 \mathrm{mmol})$ was azeotroped with benzene. $46.8 \mathrm{mg}$ of $\mathbf{4 1}(0.15 \mathrm{mmol}, 1.2$ equiv) was added and the mixture was azeotroped with benzene once more. The mixture was then dissolved in $2 \mathrm{~mL}$ of DMF and cooled to $0^{\circ} \mathrm{C} .150 \mu \mathrm{L}$ of NaHMDS (1M in THF, $0.15 \mathrm{mmol}, 1.2$ equiv) was added and the mixture was stirred for $1 \mathrm{~h} .3 \mathrm{~mL}$ of $\mathrm{H}_{2} \mathrm{O}$ was added carefully and the mixture was diluted with THF $(30 \mathrm{~mL}) .17 \mathrm{mg}$ of $\mathrm{Pd}\left(\mathrm{PPh}_{3}\right)_{4}(0.015 \mathrm{mmol}$, $10 \mathrm{~mol} \%)$ and $195 \mathrm{mg}$ of $\mathrm{Cs}_{2} \mathrm{CO}_{3}(0.6 \mathrm{mmol}, 4$ equiv) were added and the mixture was degassed and purged with $\mathrm{N}_{2}$. It was then heated to reflux for $2 \mathrm{~d}$ and extracted with saturated aqueous $\mathrm{NH}_{4} \mathrm{Cl}$ and $\mathrm{Et}_{2} \mathrm{O}(3 \mathrm{x}$ $50 \mathrm{~mL}$ ). The organic phases were dried over $\mathrm{MgSO}_{4}$ and purified via Chromatotron (hexanes:ethyl acetate 9:1 to $4: 1)$ to give the product as a pale oil $(4.3 \mathrm{mg}, 0.01 \mathrm{mmol}, 7 \%)$. $\mathrm{R}_{f}$ (hexanes:ethyl acetate $\left.4: 1\right)=0.36$. ${ }^{1} \mathrm{H}$ NMR $\left(500 \mathrm{MHz}, \mathrm{CDCl}_{3}\right): \delta[\mathrm{ppm}]=1.97-2.03(\mathrm{~m}, 1 \mathrm{H}), 2.31-2.37(\mathrm{~m}, 1 \mathrm{H}), 3.29(\mathrm{~s}, 3 \mathrm{H}), 3.60-3.65(\mathrm{~m}$, $2 \mathrm{H}), 4.36(\mathrm{dd}, J=9.9,3.4 \mathrm{~Hz}, 1 \mathrm{H}), 4.48(\mathrm{~d}, J=6.6 \mathrm{~Hz}, 1 \mathrm{H}), 4.52(\mathrm{~s}, 2 \mathrm{H}), 4.54(\mathrm{~d}, J=6.6 \mathrm{~Hz}, 1 \mathrm{H}), 4.99(\mathrm{~s}$, 
$1 \mathrm{H}), 5.16(\mathrm{~s}, 1 \mathrm{H}), 5.32(\mathrm{~d}, J=10.0 \mathrm{~Hz}, 1 \mathrm{H}), 5.71(\mathrm{t}, J=10.6 \mathrm{~Hz}, 1 \mathrm{H}), 6.13(\mathrm{~d}, J=16.0 \mathrm{~Hz}, 1 \mathrm{H}), 6.60(\mathrm{~d}, J=$ $11.4 \mathrm{~Hz}, 1 \mathrm{H}), 6.68(\mathrm{~d}, J=7.3 \mathrm{~Hz}, 1 \mathrm{H}), 6.90(\mathrm{~d}, J=8.3 \mathrm{~Hz}, 1 \mathrm{H}), 7.14(\mathrm{~d}, J=16.0 \mathrm{~Hz}, 1 \mathrm{H}), 7.23-7.25(\mathrm{~m}, 2 \mathrm{H})$, 7.29-7.36 (m, 4H) $10.54(\mathrm{~s}, 1 \mathrm{H}) .{ }^{13} \mathrm{C} \mathrm{NMR}\left(125 \mathrm{MHz}, \mathrm{CDCl}_{3}\right): \delta[\mathrm{ppm}]=29.7,55.6,67.1,72.5,73.2,75.4$, 94.1, 115.4, 116.6, 118.7, 127.5, 127.8, 128.3, 129.4, 131.5, 134.2, 134.7, 137.8, 138.4, 142.5, 142.8, 161.9, 170.5. HRMS (EI): calc. for $\mathrm{C}_{26} \mathrm{H}_{28} \mathrm{O}_{6} \mathrm{Na}\left[\mathrm{M}+\mathrm{Na}^{+}\right]$: 459.1784 ; found: 459.1763 .

2,2-Dimethyl-5-triisopropylsilanylethynyl-benzo[1,3]dioxin-4-one (30). $0.98 \mathrm{~g}$ of $\mathbf{1 0}^{[4]}$ (3.0 mmol), $1.04 \mathrm{~mL}$ of triisopropylsilylacetylene (4.5 mmol, 1.5 equiv), $29 \mathrm{mg}$ of $\mathrm{CuI}(0.15 \mathrm{mmol}$, $5 \mathrm{~mol} \%), 173 \mathrm{mg}$ of $\mathrm{Pd}\left(\mathrm{PPh}_{3}\right)_{4}(0.15 \mathrm{mmol}, 5 \mathrm{~mol} \%)$ and $0.79 \mathrm{~mL}$ of $i$ - $\operatorname{Pr}_{2} \mathrm{NEt}$ (4.5 mmol, $\left.1.5 \mathrm{equiv}\right)$ in $200 \mathrm{~mL}$ of THF were degassed and purged with $\mathrm{N}_{2}$ and the mixture was stirred for $20 \mathrm{~h}$. The reaction mixture was extracted with saturated aq. $\mathrm{NH}_{4} \mathrm{Cl} / \mathrm{Et}_{2} \mathrm{O}(3 \times 50 \mathrm{~mL})$. The organic phases were washed with brine, dried over $\mathrm{MgSO}_{4}$ and evaporated. The residue was purified by chromatography (Chromatotron, hexanes 100\% to hexanes:EtOAc 19:1) to yield a clear oil (1.04 g, $2.9 \mathrm{mmol}, 97 \%)$, which solidified upon standing. $\quad \mathrm{R}_{f}$ (hexanes:ethyl acetate $\left.6: 1\right)=0.64 . \mathrm{mp}$ (hexanes $/$ EtOAc) $=46-48{ }^{\circ} \mathrm{C}$. IR $\left(\right.$ neat): $\left[\mathrm{cm}^{-1}\right]=$ 2942, 2891, 2864, 2158, 1752, 1592, 1579, 1472, 1389, 1378, 1318, 1287, 1271, 1251, 1224, 1208, 1073, $1044,923,883,808,705 .{ }^{1} \mathrm{H}$ NMR $\left(500 \mathrm{MHz}_{,} \mathrm{CDCl}_{3}\right): \delta[\mathrm{ppm}]=1.14(\mathrm{~s}, 21 \mathrm{H}), 1.68(\mathrm{~s}, 6 \mathrm{H}), 6.88(\mathrm{~d}, J=$ $8.2 \mathrm{~Hz}, 1 \mathrm{H}), 7.26(\mathrm{~d}, J=7.7 \mathrm{~Hz}, 1 \mathrm{H}), 7.40(\mathrm{t}, J=8.0 \mathrm{~Hz}, 1 \mathrm{H}) .{ }^{13} \mathrm{C} \mathrm{NMR}\left(125 \mathrm{MHz}, \mathrm{CDCl}_{3}\right): \delta[\mathrm{ppm}]=11.4$, 18.6, 25.8, 99.6, 103.9, 105.5, 114.3, 117.2, 125.5, 129.6, 134.6, 156.5, 158.4.

(Z,Z)-(3S,4S)-2-Hydroxy-6-[(triisopropylsilanyl)-ethynyl] benzoic acid 3-(1-benzyloxy-8bromo-4-methoxymethoxy-octa-5,7-dienyl) ester (47). $262 \mathrm{mg}$ of 29 (0.7 mmol) and $301 \mathrm{mg}$ of 30 ( $0.84 \mathrm{mmol}, 1.2$ equiv) were azeotroped with benzene at $30^{\circ} \mathrm{C}$, then dissolved in $6 \mathrm{~mL}$ of $\mathrm{THF}$ at $-20^{\circ} \mathrm{C}$ and treated with $1.05 \mathrm{~mL}$ of NaHMDS (1M in THF, $1.05 \mathrm{mmol}, 1.5$ equiv). The mixture was stirred for $5 \mathrm{~h}$ while it was warming slowly to $0{ }^{\circ} \mathrm{C}$. Saturated aqueous $\mathrm{NH}_{4} \mathrm{Cl}(10 \mathrm{~mL})$ was added and the mixture was extracted with EtOAc ( 3 x $50 \mathrm{~mL}$ ). The organic phases were dried over $\mathrm{MgSO}_{4}$ and evaporated to leave a yellow oil, which was used directly without further purification. An analytical sample was purified by chromatography (Chromatotron, hexanes:EtOAc 15:1). $\mathrm{R}_{f}$ (hexanes:ethyl acetate 6:1) $=0.42 .[\alpha]^{20}{ }_{589}+11.5$ $\left(\mathrm{c}=1.33, \mathrm{CHCl}_{3}\right)$. IR (neat): $\quad\left[\mathrm{cm}^{-1}\right]=3367,3030,2942,2890,2863,2143,1739,1666,1594,1572,1496$, 1447, 1369, 1296, 1250, 1208, 1167, 1153, 1132, 1099, 1066, 1016, 919, 883, 814, 735. ${ }^{1} \mathrm{H}$ NMR $\left(500 \mathrm{MHz}_{\mathrm{CDCl}}\right): \delta[\mathrm{ppm}]=1.13(\mathrm{~s}, 21 \mathrm{H}), 1.96-2.00(\mathrm{~m}, 2 \mathrm{H}), 3.20(\mathrm{~s}, 3 \mathrm{H}), 3.50-3.56(\mathrm{~m}, 2 \mathrm{H}), 4.39(\mathrm{~d}, J=$ $11.8 \mathrm{~Hz}, 1 \mathrm{H}), 4.46(\mathrm{~d}, J=11.8 \mathrm{~Hz}, 1 \mathrm{H}), 4.48(\mathrm{~d}, J=6.9 \mathrm{~Hz}, 1 \mathrm{H}), 4.56(\mathrm{~d}, J=6.9 \mathrm{~Hz}, 1 \mathrm{H}), 4.66(\mathrm{t}, J=8.7 \mathrm{~Hz}$, $1 \mathrm{H}), 5.52(\mathrm{t}, J=10.2 \mathrm{~Hz}, 1 \mathrm{H}), 5.61-5.64(\mathrm{~m}, 1 \mathrm{H}), 6.27(\mathrm{~d}, J=7.2 \mathrm{~Hz}, 1 \mathrm{H}), 6.63(\mathrm{t}, J=10.8 \mathrm{~Hz}, 1 \mathrm{H}), 6.91(\mathrm{dd}$, $J=11.4,8.5 \mathrm{~Hz}, 1 \mathrm{H}), 6.93(\mathrm{~d}, J=8.7 \mathrm{~Hz}, 1 \mathrm{H}), 7.13(\mathrm{~d}, J=7.4 \mathrm{~Hz}, 1 \mathrm{H}), 7.22-7.32(\mathrm{~m}, 6 \mathrm{H}), 11.31(\mathrm{~s}, 1 \mathrm{H})$.

${ }^{13} \mathrm{C} \mathrm{NMR}\left(125 \mathrm{MHz}, \mathrm{CDCl}_{3}\right): \delta[\mathrm{ppm}]=11.6,18.8,30.6,55.4,66.1,72.5,73.3,73.7,93.4,97.1,107.0$, $112.3,112.3,118.4,124.7,127.2,127.6,127.8,128.3,128.3,129.6,130.2,133.7,138.0,162.2,169.2$. HRMS (EI): calcd. for $\mathrm{C}_{35} \mathrm{H}_{47} \mathrm{BrO}_{6} \mathrm{SiNa}\left[\mathrm{M}+\mathrm{Na}^{+}\right]$: 693.2223; found: 693.2240.

$(Z, Z)-(3 S, 4 S)-2-E t h y n y l-6-h y d r o x y$ benzoic acid 3-(1-benzyloxy-8-bromo-4methoxymethoxy-octa-5,7-dienyl) ester (48). Crude $47(\sim 0.7 \mathrm{mmol})$ was dissolved in $10 \mathrm{~mL}$ of $\mathrm{THF}$ and treated with $2.0 \mathrm{~mL}$ of TBAF (1M in THF, $2.0 \mathrm{mmol}, 2.8$ equiv) at $0{ }^{\circ} \mathrm{C}$. The mixture was stirred for $1.5 \mathrm{~h}$, then extracted with saturated aq. $\mathrm{NH}_{4} \mathrm{Cl} / \mathrm{EtOAc}(3 \times 50 \mathrm{~mL})$. The organic phases were dried over $\mathrm{MgSO}_{4}$ and evaporated. The residue was purified by chromatography (Chromatotron, hexanes:EtOAc 9:1) to yield a clear oil (355 mg, $0.69 \mathrm{mmol}, 98 \%$ over two steps). $\mathrm{R}_{f}$ (hexanes:ethyl acetate $\left.6: 1\right)=0.33 .[\alpha]^{20}{ }_{589}$ $-5.3\left(\mathrm{c}=0.83, \mathrm{CHCl}_{3}\right)$. IR (neat): $\left[\mathrm{cm}^{-1}\right]=3284,2890,1741,1665,1597,1571,1448,1293,1245,1209$,

[]] Fürstner, A.; Konetzki, I.Tetrahedron 1996, 52, 15071. 
1097, 1028, 919, 814. ${ }^{1} \mathrm{H}$ NMR $\left(500 \mathrm{MHz}, \mathrm{CDCl}_{3}\right): \delta[\mathrm{ppm}]=1.97-2.09(\mathrm{~m}, 2 \mathrm{H}), 3.23(\mathrm{~s}, 1 \mathrm{H}), 3.25(\mathrm{~s}, 3 \mathrm{H})$, $3.55-3.61(\mathrm{~m}, 2 \mathrm{H}), 4.44(\mathrm{~d}, J=11.8 \mathrm{~Hz}, 1 \mathrm{H}), 4.46(\mathrm{~d}, J=11.8 \mathrm{~Hz}, 1 \mathrm{H}), 4.53(\mathrm{~d}, J=6.8 \mathrm{~Hz}, 1 \mathrm{H}), 4.59(\mathrm{~d}, J=$ $6.8 \mathrm{~Hz}, 1 \mathrm{H}), 4.70(\mathrm{t}, J=8.2 \mathrm{~Hz}, 1 \mathrm{H}), 5.53-5.62(\mathrm{~m}, 2 \mathrm{H}), 6.25(\mathrm{~d}, J=7.1 \mathrm{~Hz}, 1 \mathrm{H}), 6.62(\mathrm{t}, J=10.9 \mathrm{~Hz}, 1 \mathrm{H})$, $6.91(\mathrm{dd}, J=10.4,7.4 \mathrm{~Hz}, 1 \mathrm{H}), 6.98(\mathrm{~d}, J=8.2 \mathrm{~Hz}, 1 \mathrm{H}), 7.10(\mathrm{~d}, J=7.4 \mathrm{~Hz}, 1 \mathrm{H}), 7.22-7.31(\mathrm{~m}, 5 \mathrm{H}), 7.33(\mathrm{t}$, $J=7.9 \mathrm{~Hz}, 1 \mathrm{H}), 11.23(\mathrm{~s}, 1 \mathrm{H}) .{ }^{13} \mathrm{C} \mathrm{NMR}\left(125 \mathrm{MHz}, \mathrm{CDCl}_{3}\right): \delta[\mathrm{ppm}]=30.4,55.6,66.1,72.4,73.2,74.4$, 82.4, 83.0, 93.8, 112.4, 113.1, 118.9, 123.3, 127.1, 127.2, 127.6, 127.8, 128.3, 129.7, 129.7, 134.0, 138.1, 162.2, 169.2. HRMS (EI): calc. for $\mathrm{C}_{26} \mathrm{H}_{27} \mathrm{BrO}_{6} \mathrm{Na}$ [M+Na ${ }^{+}$]: 537.0889; found: 573.0896.

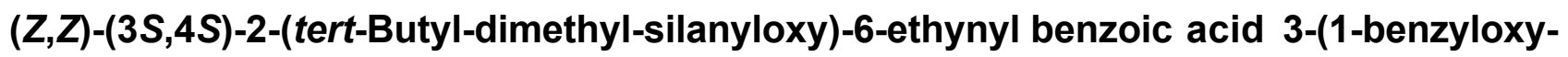
8-bromo-4-methoxymethoxy-octa-5,7-dienyl) ester (28). $355 \mathrm{mg}$ of 48 (0.69 mmol) was dissolved in $4 \mathrm{~mL}$ of DMF at $0{ }^{\circ} \mathrm{C}$. $102 \mathrm{mg}$ of imidazole (1.5 mmol, 2 equiv) and $18 \mathrm{mg}$ of DMAP (0.15 mmol, 0.2 equiv) were added followed by $345 \mu \mathrm{L}$ of TBDMSOTf $(1.5 \mathrm{mmol}, 2$ equiv) and the mixture was stirred overnight while warming to $\mathrm{rt}$. The crude reaction mixture was subjected to chromatography $(2 \mathrm{x}$ Chromatotron, first with hexanes 100\% to hexanes:EtOAc 9:1 and then with hexanes:EtOAc 19:1) to yield a clear oil (341 mg, $0.54 \mathrm{mmol}, 79 \%)$. $\mathrm{R}_{f}$ (hexanes:ethyl acetate 2:1) $=0.69$. $[\alpha]^{20}{ }_{589}+13.8\left(\mathrm{c}=0.66, \mathrm{CHCl}_{3}\right)$. IR (neat): $\quad\left[\mathrm{cm}^{-1}\right]=3286,3030,2953,2929,2887,2858,1735,1572,1460,1407,1362,1290,1257,1151$, $1111,1062,1028,919,834,784,736 .{ }^{1} \mathrm{H}$ NMR $\left(500 \mathrm{MHz}, \mathrm{CDCl}_{3}\right): \delta[\mathrm{ppm}]=0.21(\mathrm{~s}, 3 \mathrm{H}), 0.22(\mathrm{~s}, 3 \mathrm{H})$, $0.97(\mathrm{~s}, 9 \mathrm{H}), 2.07-2.09(\mathrm{~m}, 1 \mathrm{H}), 2.15-2.18(\mathrm{~m}, 1 \mathrm{H}), 3.28(\mathrm{~s}, 3 \mathrm{H}), 3.35(\mathrm{~s}, 1 \mathrm{H}), 3.65-3.71(\mathrm{~m}, 2 \mathrm{H}), 4.47(\mathrm{~d}, J=$ $11.9 \mathrm{~Hz}, 1 \mathrm{H}), 4.52(\mathrm{~d}, J=6.8 \mathrm{~Hz}, 1 \mathrm{H}), 4.53(\mathrm{~d}, J=11.9 \mathrm{~Hz}, 1 \mathrm{H}), 4.56(\mathrm{~d}, J=6.9 \mathrm{~Hz}, 1 \mathrm{H}), 4.68(\mathrm{dd}, J=9.1$, $4.6 \mathrm{~Hz}, 1 \mathrm{H}), 5.31-5.33(\mathrm{~m}, 1 \mathrm{H}), 5.67(\mathrm{t}, J=10.1 \mathrm{~Hz}, 1 \mathrm{H}), 6.16(\mathrm{~d}, J=7.1 \mathrm{~Hz}, 1 \mathrm{H}), 6.55(\mathrm{t}, J=11.0 \mathrm{~Hz}, 1 \mathrm{H})$, $6.84(\mathrm{~d}, J=8.2 \mathrm{~Hz}, 1 \mathrm{H}), 6.91(\mathrm{dd}, J=10.4,7.4 \mathrm{~Hz}, 1 \mathrm{H}), 7.08(\mathrm{~d}, J=7.5 \mathrm{~Hz}, 1 \mathrm{H}), 7.17(\mathrm{t}, J=8.0 \mathrm{~Hz}, 1 \mathrm{H})$,

7.26-7.33 (m, 5H). ${ }^{13} \mathrm{C}$ NMR $\left(125 \mathrm{MHz}, \mathrm{CDCl}_{3}\right): \delta[\mathrm{ppm}]=-4.3,-4.1,18.4,25.8,30.7,55.6,66.9,71.6,73.1$, $74.3,80.8,81.0,93.7,111.6,120.3,120.8,125.5,125.7,127.5,127.6,127.8,128.4,128.9,130.0,130.5,138.4$, 152.5, 166.2. HRMS (EI): calcd. for $\mathrm{C}_{32} \mathrm{H}_{41} \mathrm{BrO}_{6} \mathrm{SiNa}$ [M+ Na ${ }^{+}$: 651.1753; found: 651.1735 .

Potassium (2E,5Z,7Z)-(3S,4S)-2-\{2-[3-(1-Benzyloxy-8-bromo-4-methoxymethoxy-octa5,7-dienyl)]-3-hydroxy-phenyl\}-vinyltrifluoroborate (27). 3.15 mL of 2,5-dimethylhexa-2,4-diene $(22 \mathrm{mmol})$ in $17 \mathrm{~mL}$ of THF was treated with $5 \mathrm{~mL}$ of $\mathrm{BH}_{3} \cdot \mathrm{SMe}_{2}(2 \mathrm{M}$ in toluene, $10 \mathrm{mmol})$ at $0{ }^{\circ} \mathrm{C}$. The mixture was stirred for $3 \mathrm{~h}$ to give a clear, $\sim 0.4 \mathrm{M}$ solution of $i-\mathrm{PP}_{2} \mathrm{BH} .215 \mathrm{mg}$ of $28(0.34 \mathrm{mmol})$ in $3 \mathrm{~mL}$ of THF was stirred at $\mathrm{rt}$ and $1.3 \mathrm{~mL}$ of $i-\mathrm{PP}_{2} \mathrm{BH}(0.4 \mathrm{M}$ in THF, $0.52 \mathrm{mmol}, 0.6$ equiv) was added. The reaction was monitored by TLC and after disappearance of the staring material $(0.5 \mathrm{~h}), 0.5 \mathrm{~mL}$ of $\mathrm{H}_{2} \mathrm{O}$ was added carefully at $0{ }^{\circ} \mathrm{C}$. After an additional $1.5 \mathrm{~h}, 2.5 \mathrm{~mL}$ of $\mathrm{CH}_{2} \mathrm{O}\left(37 \%\right.$ in $\left.\mathrm{H}_{2} \mathrm{O}\right)$ was added. The mixture was stirred overnight, extracted with brine/EtOAc $(3 \times 50 \mathrm{~mL})$ and the organic phases were evaporated. The residue was dissolved in $15 \mathrm{~mL}$ of acetone/MeCN (2:1) and $0.53 \mathrm{~g}$ of $\mathrm{KHF}_{2}$ (6.8 mmol, 20 equiv) in $5 \mathrm{~mL}$ of $\mathrm{H}_{2} \mathrm{O}$ was added. The mixture was stirred for $4 \mathrm{~h}$ at $\mathrm{rt}$, evaporated and the residue was extracted with hot acetone $(3 \times 20 \mathrm{~mL})$ and $\mathrm{MeCN}(20 \mathrm{~mL})$. The organic phases were evaporated and the residue was dried in vacuo at $50{ }^{\circ} \mathrm{C}$ overnight to yield the slightly impure product as a pale wax $(249 \mathrm{mg},>99 \%)$. ${ }^{1} \mathrm{H} \mathrm{NMR}$ $\left(500 \mathrm{MHz}\right.$, acetone- $\left.d_{6}\right): \delta[\mathrm{ppm}]=2.00-2.09(\mathrm{~m}, 2 \mathrm{H}), 3.24(\mathrm{~s}, 3 \mathrm{H}), 3.64-3.70(\mathrm{~m}, 2 \mathrm{H}), 4.50(\mathrm{~s}, J=2 \mathrm{H}), 4.61$ $(\mathrm{m}, 2 \mathrm{H}), 4.82(\mathrm{dd}, J=9.4,6.8 \mathrm{~Hz}, 1 \mathrm{H}), 5.48-5.53(\mathrm{~m}, 1 \mathrm{H}), 5.75(\mathrm{t}, J=10.4 \mathrm{~Hz}, 1 \mathrm{H}), 6.16\left(\mathrm{dq}, J_{\mathrm{H}-\mathrm{H}}=17.9 \mathrm{~Hz}\right.$, $\left.J_{\mathrm{H}-\mathrm{F}}=3.6 \mathrm{~Hz}, 1 \mathrm{H}\right), 6.46(\mathrm{~d}, J=7.0 \mathrm{~Hz}, 1 \mathrm{H}), 6.58(\mathrm{t}, J=10.9 \mathrm{~Hz}, 1 \mathrm{H}), 6.73(\mathrm{~d}, J=8.0 \mathrm{~Hz}, 1 \mathrm{H}), 7.04(\mathrm{~d}, J=$ $7.6 \mathrm{~Hz}, 1 \mathrm{H}), 7.11(\mathrm{~d}, J=8.0 \mathrm{~Hz}, 1 \mathrm{H}), 7.17(\mathrm{t}, J=17.9 \mathrm{~Hz}, 1 \mathrm{H}), 7.21(\mathrm{dd}, J=10.8,7.5 \mathrm{~Hz}, 1 \mathrm{H}), 7.22-7.26(\mathrm{~m}$, $2 \mathrm{H}), 7.27-7.31(\mathrm{~m}, 2 \mathrm{H}), 7.33-7.36(\mathrm{~m}, 2 \mathrm{H}), 10.5(\mathrm{brs}, 1 \mathrm{H}) .{ }^{13} \mathrm{C}$ NMR $\left(125 \mathrm{MHz}\right.$, acetone- $\left.d_{6}\right): \delta[\mathrm{ppm}]=31.3$, 55.7, 67.2 , 73.4, 73.9, 74.4, 95.0, 112.3, 114.7, 116.1, 118.7, 128.0, 128.5, 128.8, 129.0, 129.4, 132.2, 132.9, 134.1, 139.9, 144.6, 159.2, 170.4. ${ }^{19}$ F NMR (470 MHz, acetone- $\left.d_{6}\right): \delta[\mathrm{ppm}]=-142.0(\mathrm{~s})$.

$(9 Z, 11 Z, 13 E)-(7 S, 8 S)-7-(2-B e n z y l o x y-e t h y l)-4-(h y d r o x y)-8-m e t h o x y m e t h o x y-7,8-$ dihydro-6-oxa-benzocyclododecen-5-one (49). $114 \mathrm{mg}$ of $27(0.156 \mathrm{mmol})$ was dissolved in $170 \mathrm{~mL}$ 
of $\mathrm{THF} / \mathrm{H}_{2} \mathrm{O}(10: 1), 390 \mathrm{mg}$ of $\mathrm{Cs}_{2} \mathrm{CO}_{3}\left(0.65 \mathrm{mmol}, 5\right.$ equiv) and $20 \mathrm{mg}$ of $\mathrm{Pd}\left(\mathrm{PPh}_{3}\right)_{4}(0.017 \mathrm{mmol}$, $10 \mathrm{~mol} \%$ ) were added, the mixture was degassed and purged with $\mathrm{N}_{2}$ and then heated to reflux for $20 \mathrm{~h}$. After cooling to rt, the mixture was extracted with saturated $\mathrm{NH}_{4} \mathrm{Cl} / \mathrm{EtOAc}(3 \times 100 \mathrm{~mL})$, the organic phases were dried over $\mathrm{MgSO}_{4}$ and purified by chromatography (Chromatotron, hexanes:EtOAc 4:1) to yield a clear oil $\left(28.5 \mathrm{mg}, 0.065 \mathrm{mmol}, 42 \%\right.$ over 2 steps). $\quad \mathrm{R}_{f}$ (hexanes:ethyl acetate $\left.2: 1\right)=0.37 .[\alpha]^{20}{ }_{589}-137.7$ $\left(\mathrm{c}=0.57, \mathrm{CHCl}_{3}\right)$. IR (neat): $\quad\left[\mathrm{cm}^{-1}\right]=3315,3011,2925,1730,1662,1604,1575,1495,1463,1423,1363$, 1326, 1294, 1247, 1216, 1155, 1098, 1056, 1029, 1000, 955, 918, 852, 804, 764, 738. ${ }^{1} \mathrm{H}$ NMR $(500 \mathrm{MHz}$, $\left.\mathrm{CDCl}_{3}\right): \delta[\mathrm{ppm}]=2.11-2.18(\mathrm{~m}, 2 \mathrm{H}), 3.22(\mathrm{~s}, 3 \mathrm{H}), 3.35(\mathrm{~s}, 1 \mathrm{H}), 3.63-3.68(\mathrm{~m}, 2 \mathrm{H}), 4.48(\mathrm{~d}, J=6.6 \mathrm{~Hz}, 1 \mathrm{H})$, $4.55(\mathrm{~s}, 2 \mathrm{H}), 4.55(\mathrm{~d}, J=6.6 \mathrm{~Hz}, 1 \mathrm{H}), 4.65(\mathrm{dd}, J=10.3,3.7 \mathrm{~Hz}, 1 \mathrm{H}), 5.45-5.48(\mathrm{~m}, 1 \mathrm{H}), 5.64(\mathrm{t}, J=10.2 \mathrm{~Hz}$, $1 \mathrm{H}), 5.87$ (dd, $J=10.1,3.7 \mathrm{~Hz}, 1 \mathrm{H}), 6.19(\mathrm{dd}, J=11.8,3.2 \mathrm{~Hz}, 1 \mathrm{H}), 6.31(\mathrm{t}, J=10.8 \mathrm{~Hz}, 1 \mathrm{H}), 6.71-6.76(\mathrm{~m}$, $3 \mathrm{H}), 6.78(\mathrm{~d}, J=8.2 \mathrm{~Hz}, 1 \mathrm{H}), 7.19(\mathrm{t}, J=8.0 \mathrm{~Hz}, 1 \mathrm{H}), 7.26-7.31(\mathrm{~m}, 1 \mathrm{H}), 7.32-7.35(\mathrm{~m}, 4 \mathrm{H}) .{ }^{13} \mathrm{C}$ NMR $\left(125 \mathrm{MHz}, \mathrm{DMSO}-d_{6}\right): \delta[\mathrm{ppm}]=27.6,54.7,65.7,67.9,72.1,73.3,92.4,114.2,118.6,121.8,126.8,127.3$, 127.5, 127.8, 128.2, 129.5, 130.0, 130.2, 131.8, 132.2, 137.4, 138.5, 153.9, 168.0. (Note: In other solvents $\left(\mathrm{CDCl}_{3}, \mathrm{C}_{6} \mathrm{D}_{6}\right.$ or acetone- $\left.d_{6}\right)$, many signals broaden at rt.) HRMS (EI): calc. for $\mathrm{C}_{26} \mathrm{H}_{28} \mathrm{O}_{6} \mathrm{Na}\left[\mathrm{M}+\mathrm{Na}^{+}\right]$: 459.1784; found: 459.1776 .

\section{(9Z,11Z,13E)-(7S,8S)-7-(2-Benzyloxy-ethyl)-4-(tert-butyl-dimethyl-silanyloxy)-8-} methoxymethoxy-7,8-dihydro-6-oxa-benzocyclododecen-5-one (50). $8.1 \mathrm{mg}$ of $\mathbf{4 9}$ (0.019 mmol) in $0.7 \mathrm{~mL}$ of DMF were treated at $\mathrm{rt}$ with $30 \mathrm{mg}$ of TBDMSCl $(0.19 \mathrm{mmol}, 10$ equiv) and $13 \mathrm{mg}$ of imidazole $(0.19 \mathrm{mmol}, 10$ equiv). The mixture was stirred for $16 \mathrm{~h}$. The mixture was then extracted with saturated $\mathrm{NH}_{4} \mathrm{Cl}$ solution and $\mathrm{Et}_{2} \mathrm{O}(4 \times 20 \mathrm{~mL})$, the organic phases were dried over $\mathrm{MgSO}_{4}$ and evaporated. Purification via chromatography (silica gel, hexanes:EtOAc 19:1) gave the product as a clear oil $(9.0 \mathrm{mg}$, $0.016 \mathrm{mmol}, 86 \%) . \mathrm{R}_{f}$ (hexanes:ethyl acetate $\left.4: 1\right)=0.56 .[\alpha]^{20}{ }_{589}-105.3\left(\mathrm{c}=0.66, \mathrm{CHCl}_{3}\right) . \mathrm{IR}$ (neat): $\quad\left[\mathrm{cm}^{-}\right.$ $\left.{ }^{1}\right]=2928,2858,1730,1670,1637,1571,1555,1462,1364,1292,1254,1152,1102,1062,1031,1000$, 955, 918, 840, 807, 775, 732. ${ }^{1} \mathrm{H}$ NMR $\left(500 \mathrm{MHz}, \mathrm{CDCl}_{3}\right): \delta[\mathrm{ppm}]=0.18(\mathrm{~s}, 3 \mathrm{H}), 0.19(\mathrm{~s}, 3 \mathrm{H}), 0.96(\mathrm{~s}$, $9 \mathrm{H}), 2.05-2.11(\mathrm{~m}, 1 \mathrm{H}), 2.16-2.23(\mathrm{~m}, 1 \mathrm{H}), 3.33(\mathrm{~s}, 3 \mathrm{H}), 3.65-3.82(\mathrm{~m}, 2 \mathrm{H}), 3.84-3.88(\mathrm{~m}, 1 \mathrm{H}), 4.49-4.55(\mathrm{~m}$, $4 \mathrm{H}), 4.60(\mathrm{dd}, J=10.7,2.6 \mathrm{~Hz}, 1 \mathrm{H}), 5.39-5.42(\mathrm{~m}, 1 \mathrm{H}), 5.65(\mathrm{t}, J=11.2 \mathrm{~Hz}, 1 \mathrm{H}), 5.86(\mathrm{dd}, J=11.0,3.9 \mathrm{~Hz}$, $1 \mathrm{H}), 6.18(\mathrm{dd}, J=11.9,3.9 \mathrm{~Hz}, 1 \mathrm{H}), 6.33(\mathrm{t}, J=11.1 \mathrm{~Hz}, 1 \mathrm{H}), 6.62(\mathrm{~d}, J=16.0 \mathrm{~Hz}, 1 \mathrm{H}), 6.73(\mathrm{~d}, J=8.1 \mathrm{~Hz}$, $1 \mathrm{H}), 6.77(\mathrm{~d}, J=7.6 \mathrm{~Hz}, 1 \mathrm{H}), 6.99(\mathrm{dd}, J=16.0,11.4 \mathrm{~Hz}, 1 \mathrm{H}), 7.15(\mathrm{t}, J=8.0 \mathrm{~Hz}, 1 \mathrm{H}), 7.25-7.28(\mathrm{~m}, 1 \mathrm{H})$, 7.30-7.35 (m, 4H). ${ }^{13} \mathrm{C}$ NMR $\left(125 \mathrm{MHz}, \mathrm{CDCl}_{3}\right): \delta[\mathrm{ppm}]=-4.2,-4.1,18.4,26.0,29.2,55.4,67.8,68.8,77.8$, 75.3, 93.2, 118.0, 121.3, 126.4, 126.9, 127.2, 127.5, 127.5, 128.3, 129.3, 130.0, 131.0, 132.1, 132.2, 137.9, 138.5, 152.3, 168.2. HRMS (EI): calc. for $\mathrm{C}_{32} \mathrm{H}_{42} \mathrm{O}_{6} \mathrm{SiNa}$ [M+Na ${ }^{+}$]: 573.2648; found: 573.2632.

(9Z,11Z,13E)-(7S,8S)- 4-(tert-Butyl-dimethyl-silanyloxy)-7-(2-hydroxy-ethyl)-8methoxymethoxy-7,8-dihydro-6-oxa-benzocyclododecen-5-one (6). $18.6 \mathrm{mg}$ of $\mathbf{5 0}$ (0.033 mmol) was dissolved in $2 \mathrm{~mL}$ ofCH $\mathrm{Cl}_{2} /$ buffer-solution $\mathrm{pH}=7$ and $150 \mathrm{mg}$ of DDQ ( $0.66 \mathrm{mmol}, 20$ equiv) were added. The mixture was stirred at $\mathrm{rt}$ overnight, then heated to reflux for $6 \mathrm{~h}$ and stirred at $\mathrm{rt}$ again overnight. The mixture was then extracted with saturated aqueous $\mathrm{NaHCO}_{3}$ and $\mathrm{CH}_{2} \mathrm{Cl}_{2}(4 \times 25 \mathrm{~mL})$. The organic phases were dried over $\mathrm{MgSO}_{4}$ and evaporated. Chromatographic purification (silica gel hexanes:EtOAc 6:1 to $3: 1)$ yielded a clear oil $(11.6 \mathrm{mg}, 0.025 \mathrm{mmol}, 77 \%) . \mathrm{R}_{f}$ (hexanes:ethyl acetate $\left.4: 1\right)=0.16 .[\alpha]^{20}{ }_{589}-153.2$ $\left(\mathrm{c}=0.65, \mathrm{CHCl}_{3}\right)$. IR (neat): $\left[\mathrm{cm}^{-1}\right]=3434,2931,2858,1828,1769,1730,1633,1572,1463,1454,1257$, $1152,1101,1047,949,913,839,807,786,740 .{ }^{1} \mathrm{H}$ NMR $\left(500 \mathrm{MHz}, \mathrm{CDCl}_{3}\right): \delta[\mathrm{ppm}]=0.20(\mathrm{~s}, 3 \mathrm{H}), 0.21$ (s, 3H), $0.97(\mathrm{~s}, 9 \mathrm{H}), 2.04-2.08(\mathrm{~m}, 1 \mathrm{H}), 2.20(\mathrm{bt}, J=5.8 \mathrm{~Hz}, 1 \mathrm{H}), 2.22-2.26(\mathrm{~m}, 1 \mathrm{H}), 3.36(\mathrm{~s}, 3 \mathrm{H}), 3.80-3.83$ $(\mathrm{m}, 1 \mathrm{H}), 3.84-3.88(\mathrm{~m}, 1 \mathrm{H}), 4.53(\mathrm{~d}, J=6.5 \mathrm{~Hz}, 1 \mathrm{H}), 4.57(\mathrm{~d}, J=6.5 \mathrm{~Hz}, 1 \mathrm{H}), 4.65(\mathrm{dd}, J=10.7,2.9 \mathrm{~Hz}, 1 \mathrm{H})$, $5.44-5.48(\mathrm{~m}, 1 \mathrm{H}), 5.72(\mathrm{t}, J=11.3 \mathrm{~Hz}, 1 \mathrm{H}), 5.86(\mathrm{dd}, J=11.1,4.1 \mathrm{~Hz}, 1 \mathrm{H}), 6.20(\mathrm{dd}, J=11.9,4.1 \mathrm{~Hz}, 1 \mathrm{H})$, $6.34(\mathrm{t}, J=11.4 \mathrm{~Hz}, 1 \mathrm{H}), 6.64(\mathrm{~d}, J=16.0 \mathrm{~Hz}, 1 \mathrm{H}), 6.73(\mathrm{~d}, J=8.1 \mathrm{~Hz}, 1 \mathrm{H}), 6.78(\mathrm{~d}, J=7.7 \mathrm{~Hz}, 1 \mathrm{H}), 6.98$ 
$(\mathrm{dd}, J=16.0,11.4 \mathrm{~Hz}, 1 \mathrm{H}), 7.15(\mathrm{t}, J=8.0 \mathrm{~Hz}, 1 \mathrm{H}) .{ }^{13} \mathrm{C} \mathrm{NMR}\left(125 \mathrm{MHz}, \mathrm{CDCl}_{3}\right): \delta[\mathrm{ppm}]=-4.2,18.4$, 25.8, 32.0, 55.6, 60.2, 68.7, 76.4, 93.3, 117.9, 121.3, 126.3, 126.6, 127.2, 129.4, 130.0, 131.0, 132.4, 132.7, 138.1, 152.3, 168.2. HRMS (EI): calc. for $\mathrm{C}_{25} \mathrm{H}_{36} \mathrm{O}_{6} \mathrm{NaSi}\left[\mathrm{M}+\mathrm{Na}^{+}\right]$: 483.2179; found: 483.2184 . 
$\mathrm{Br}$

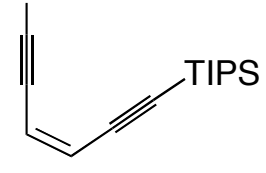

17

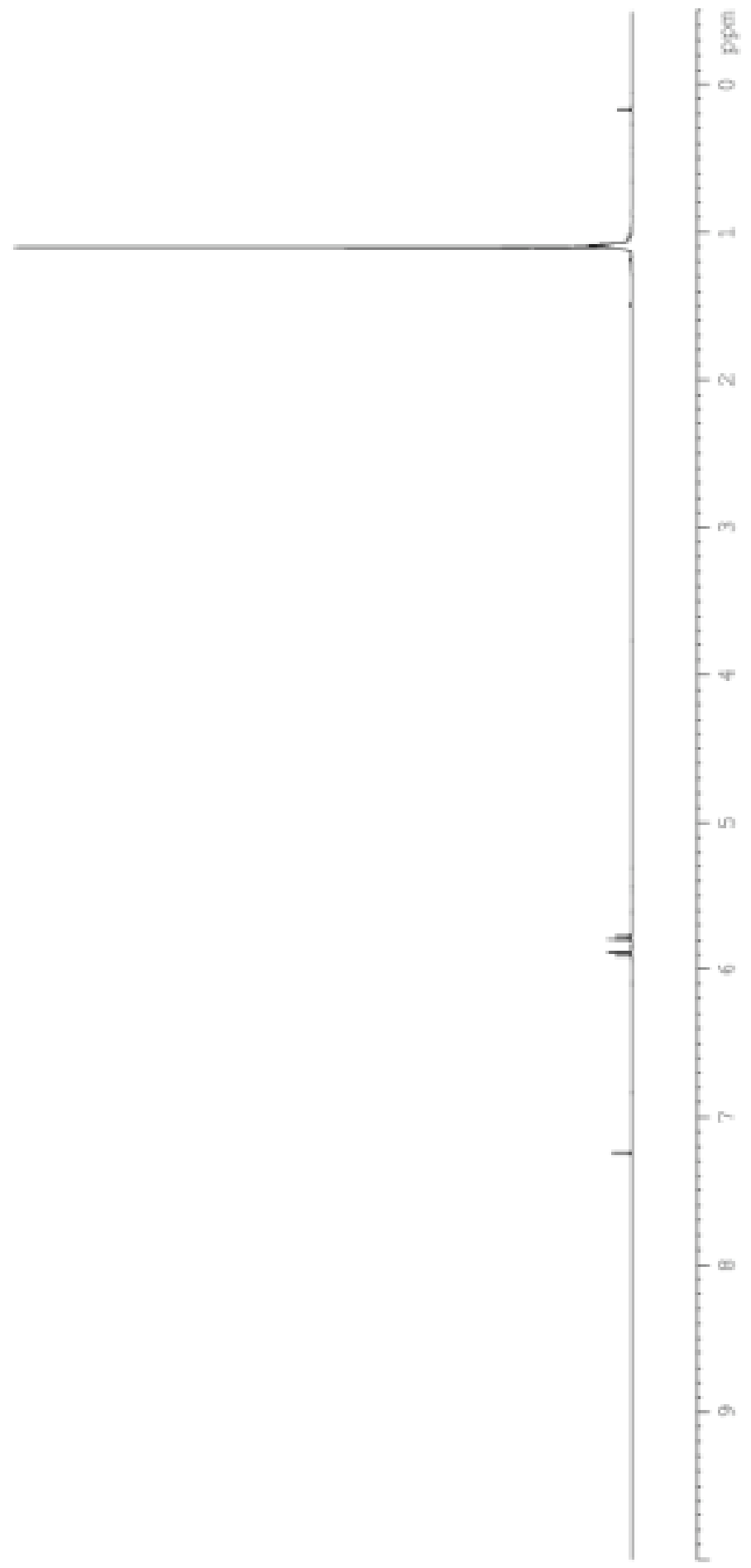

S14 

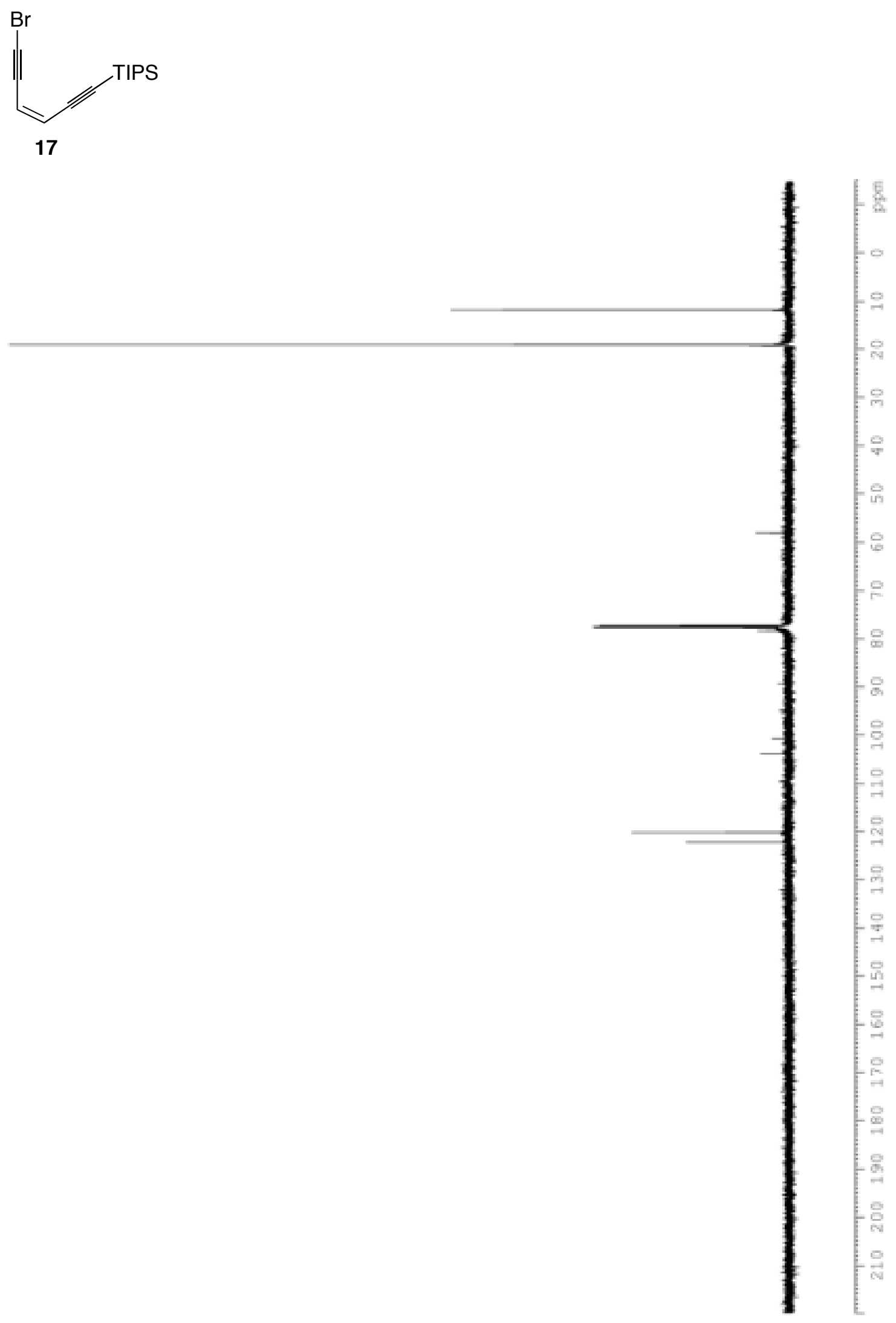

S15 


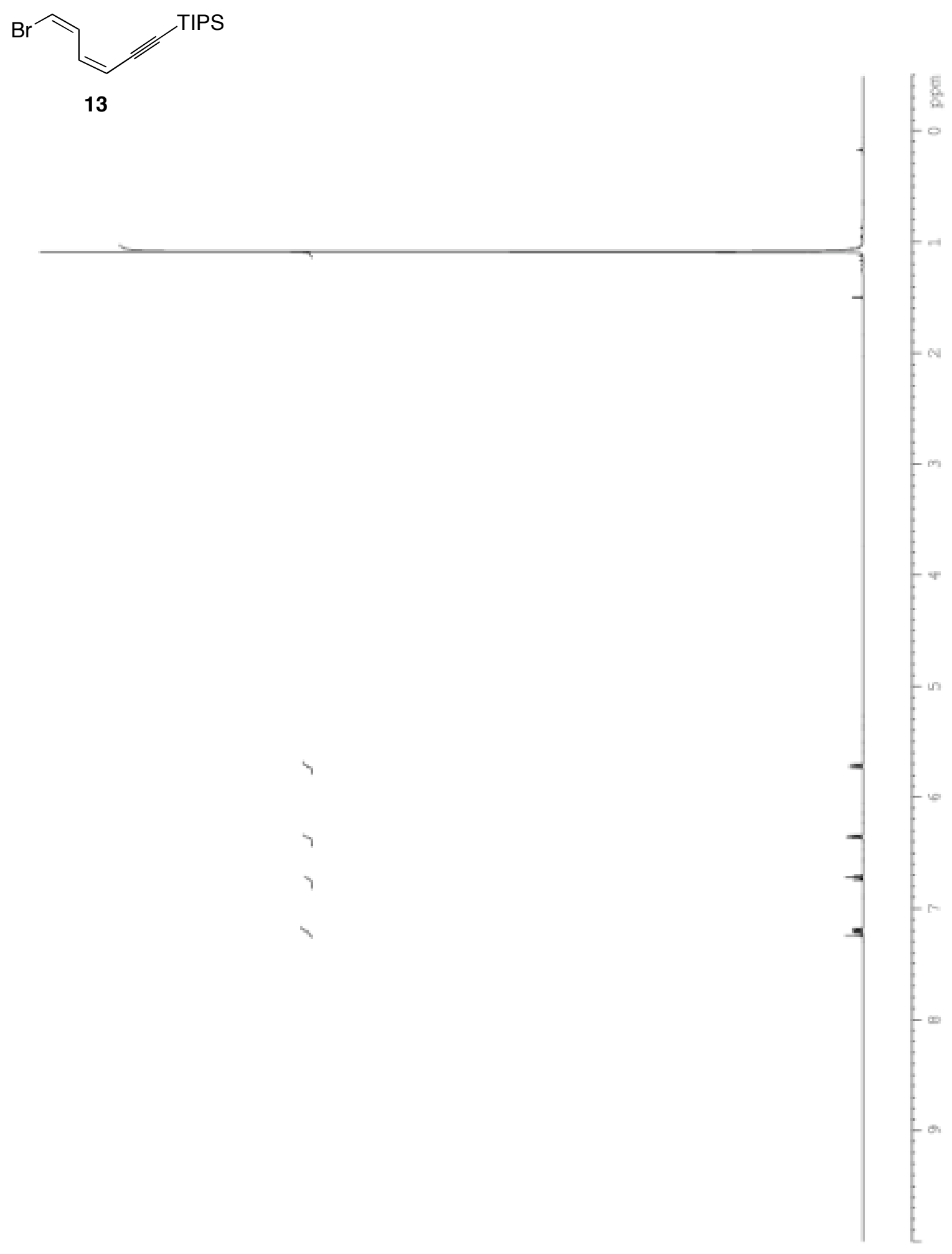



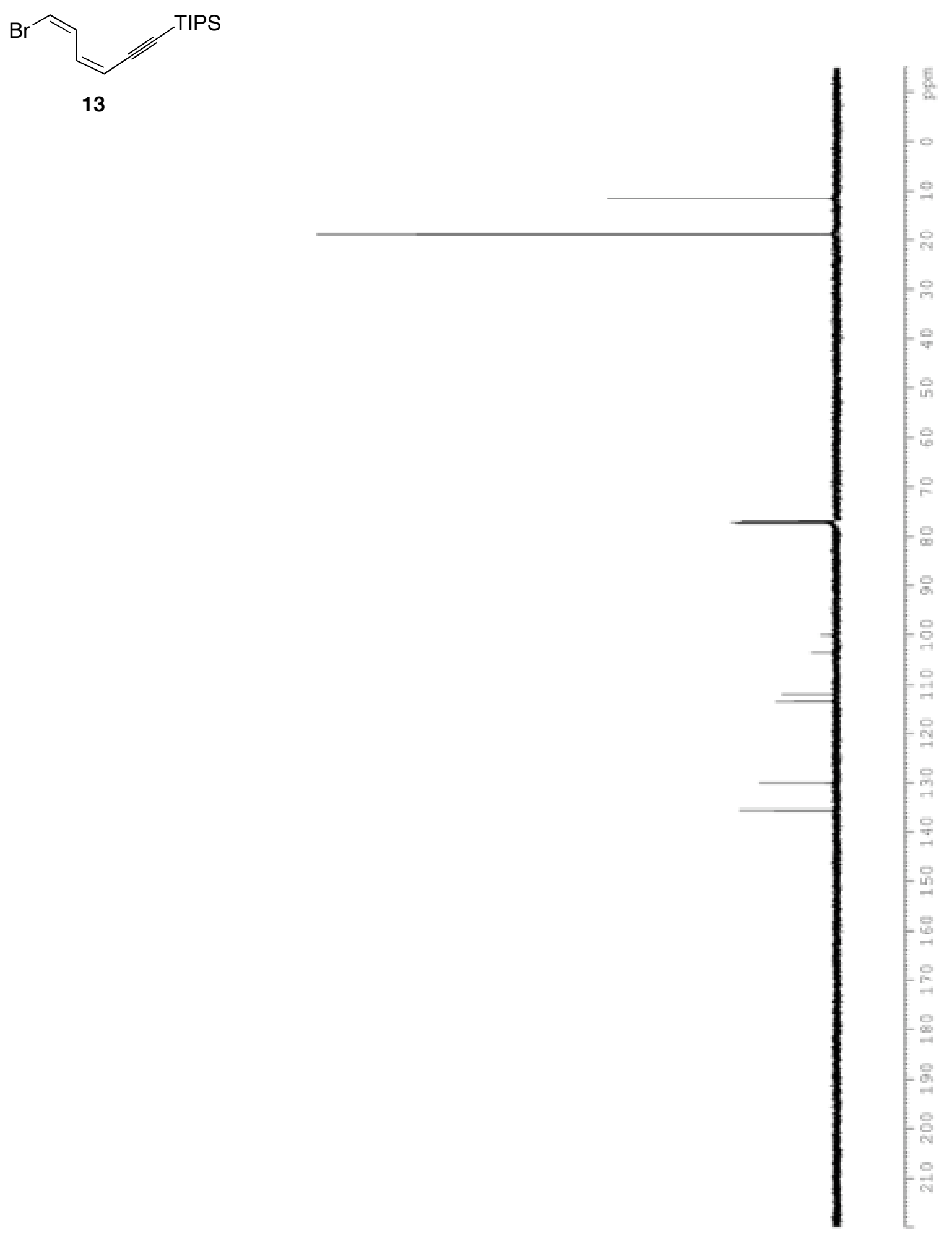


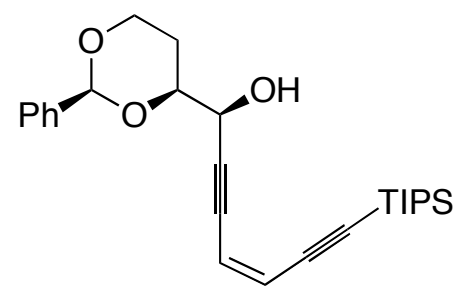

19

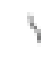

$\checkmark$

4

5

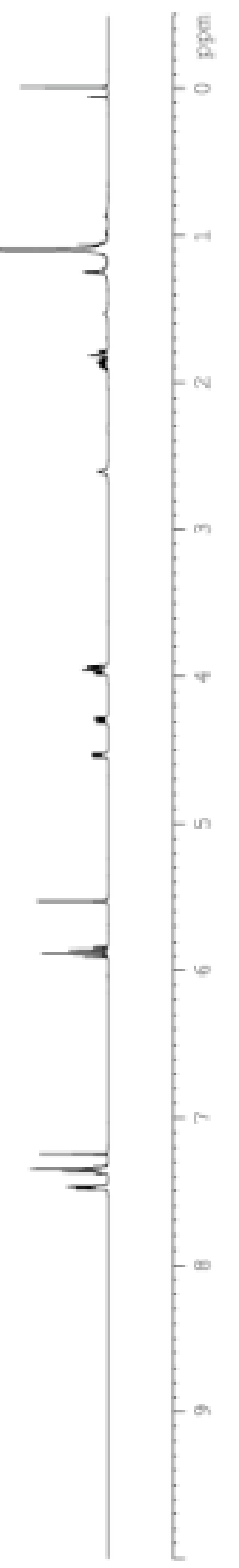



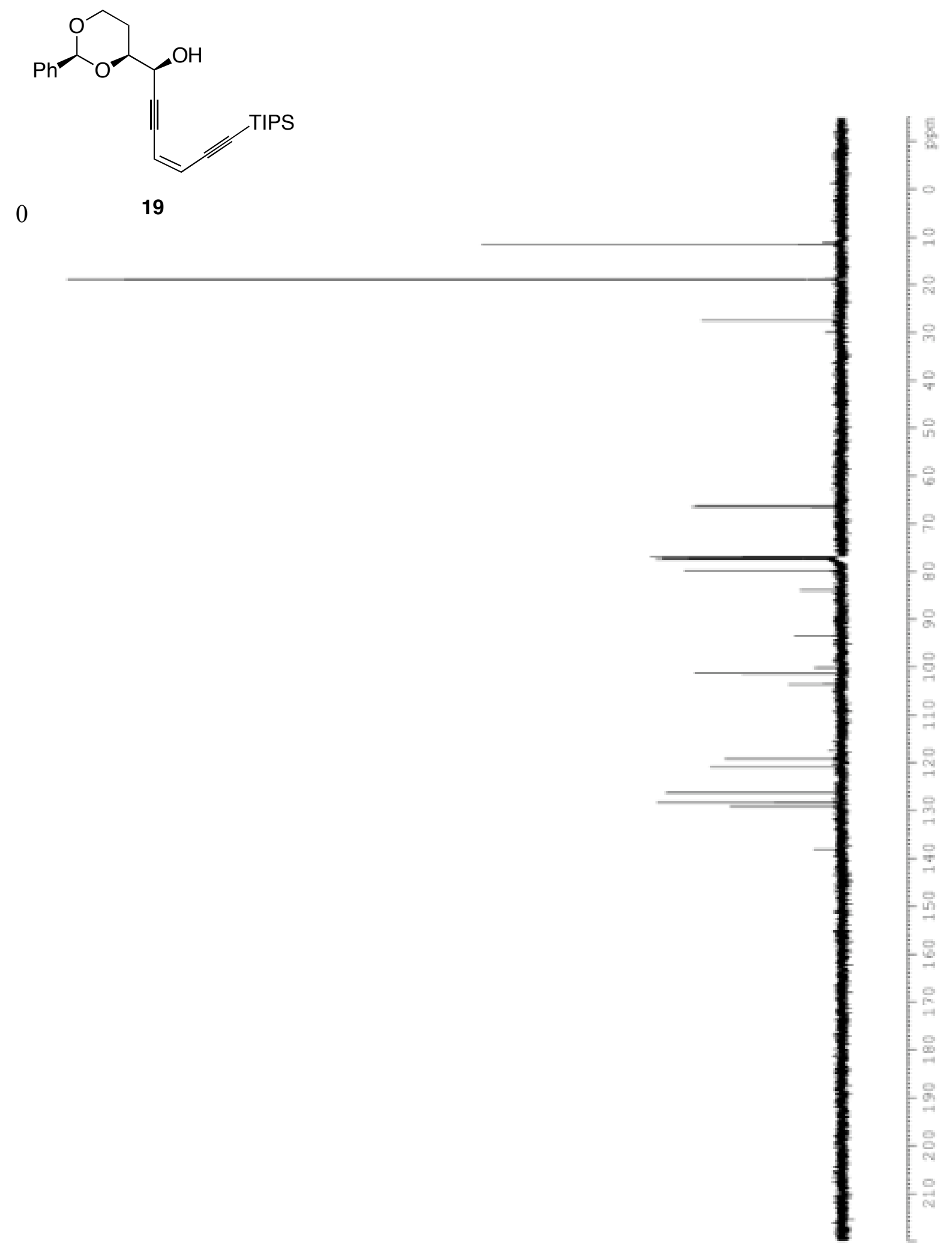

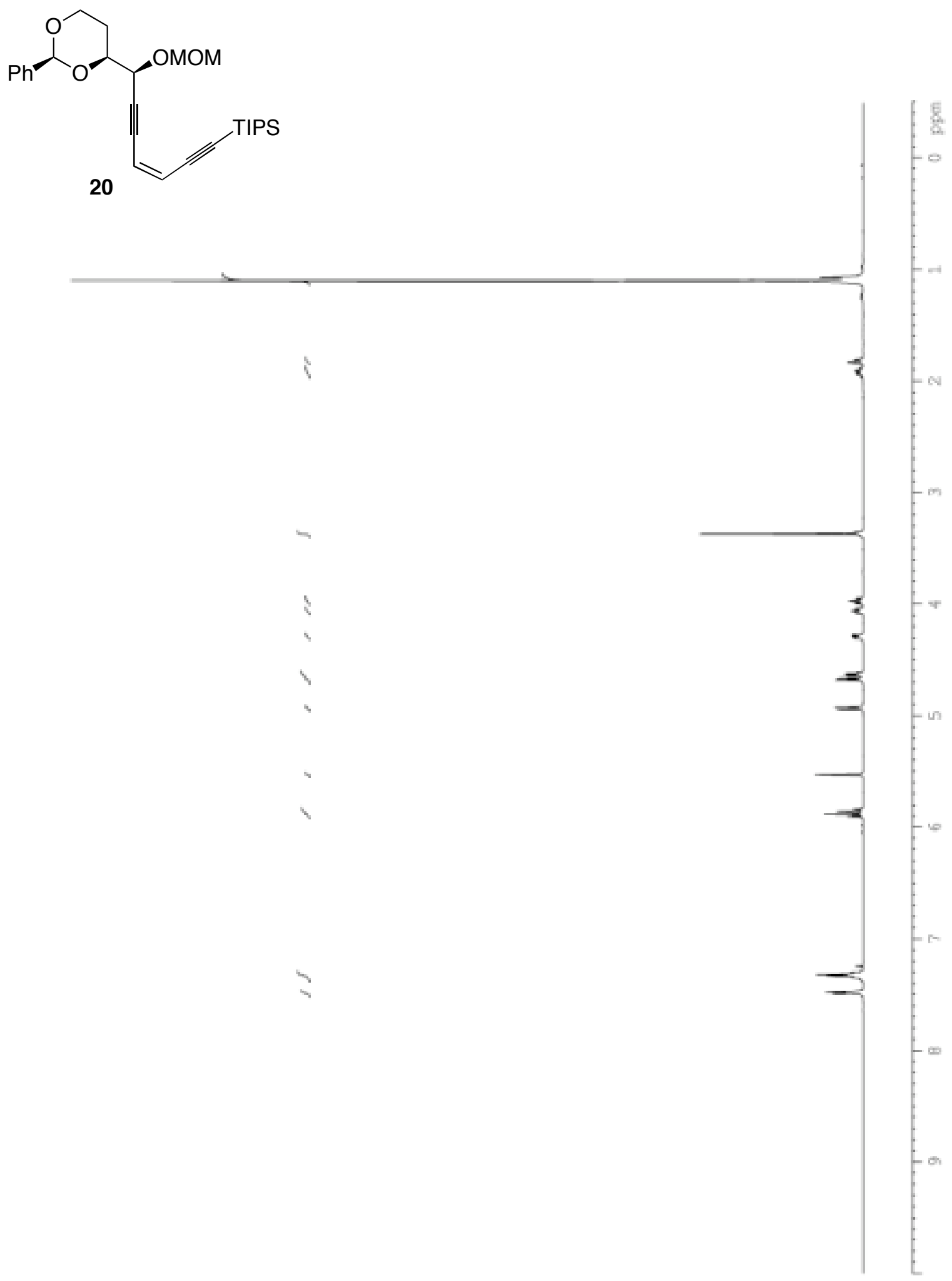

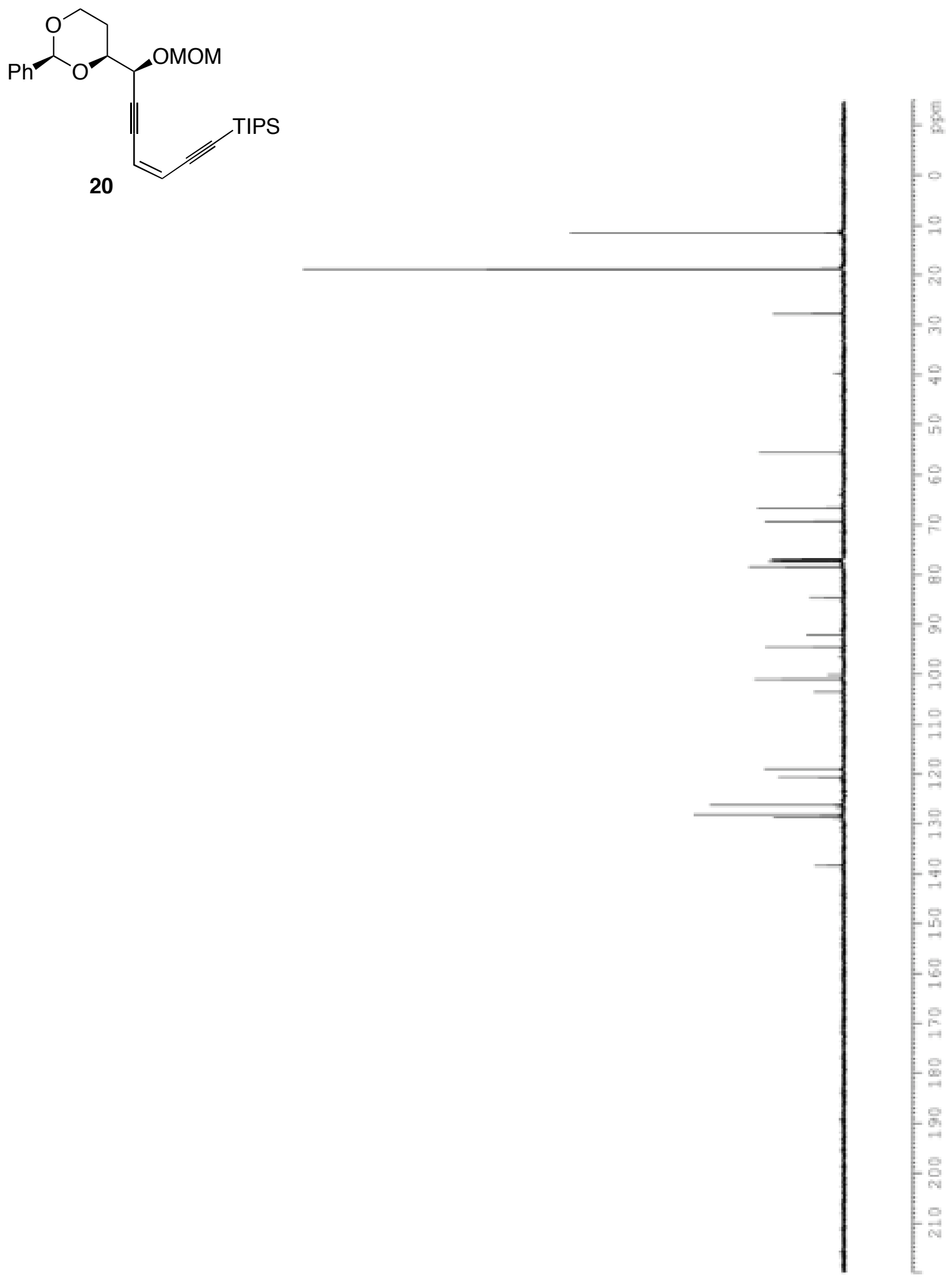

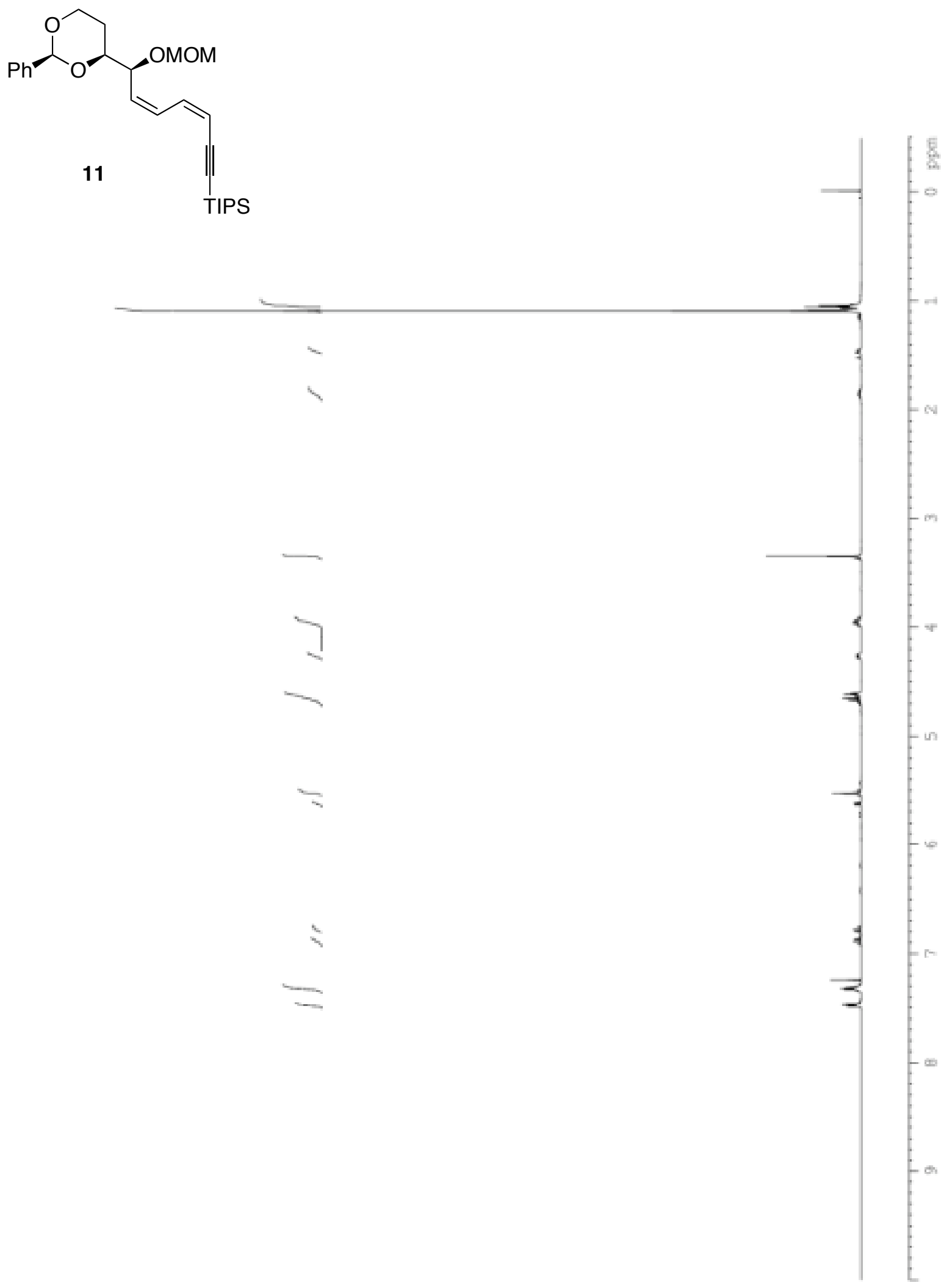

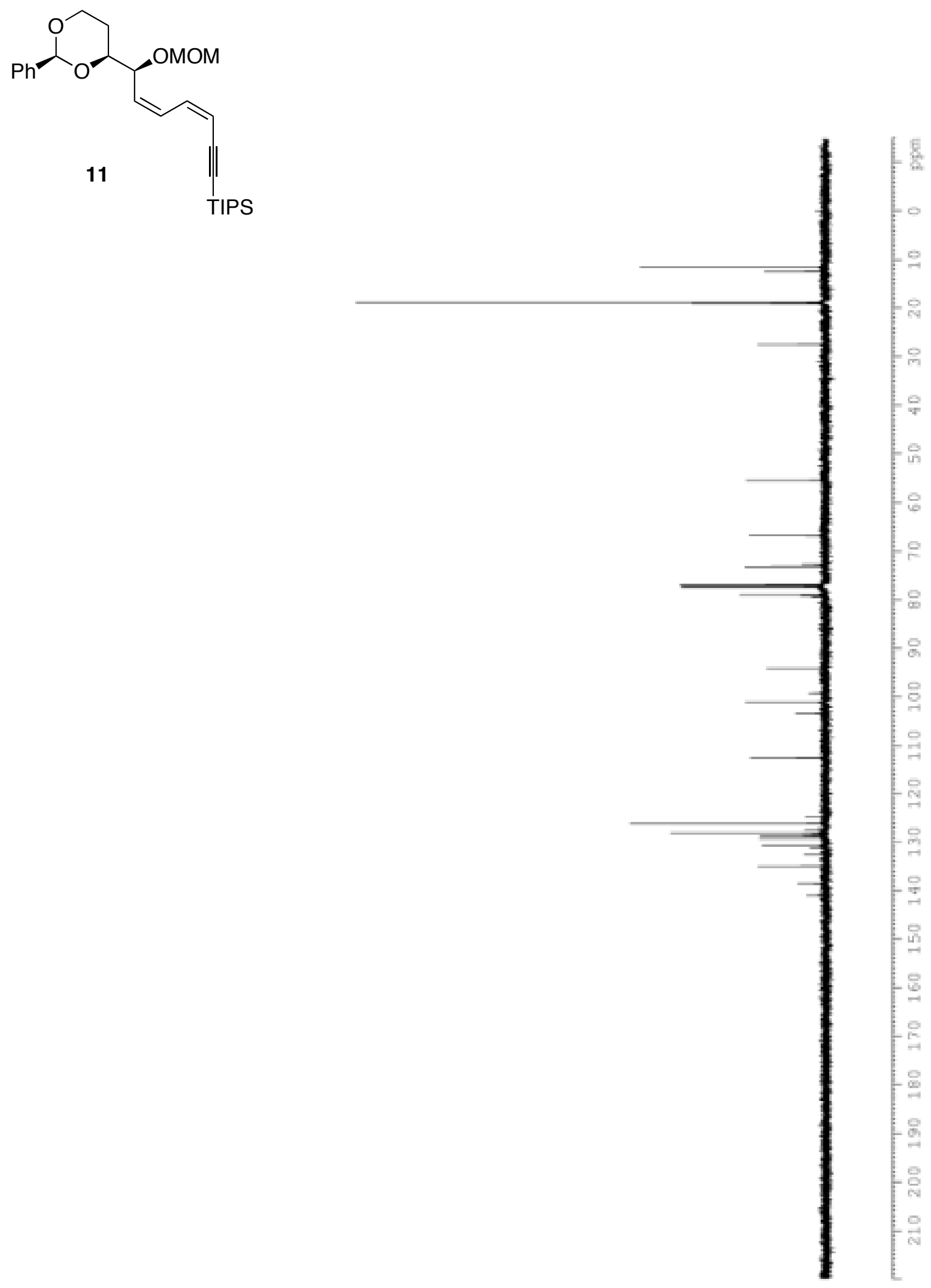

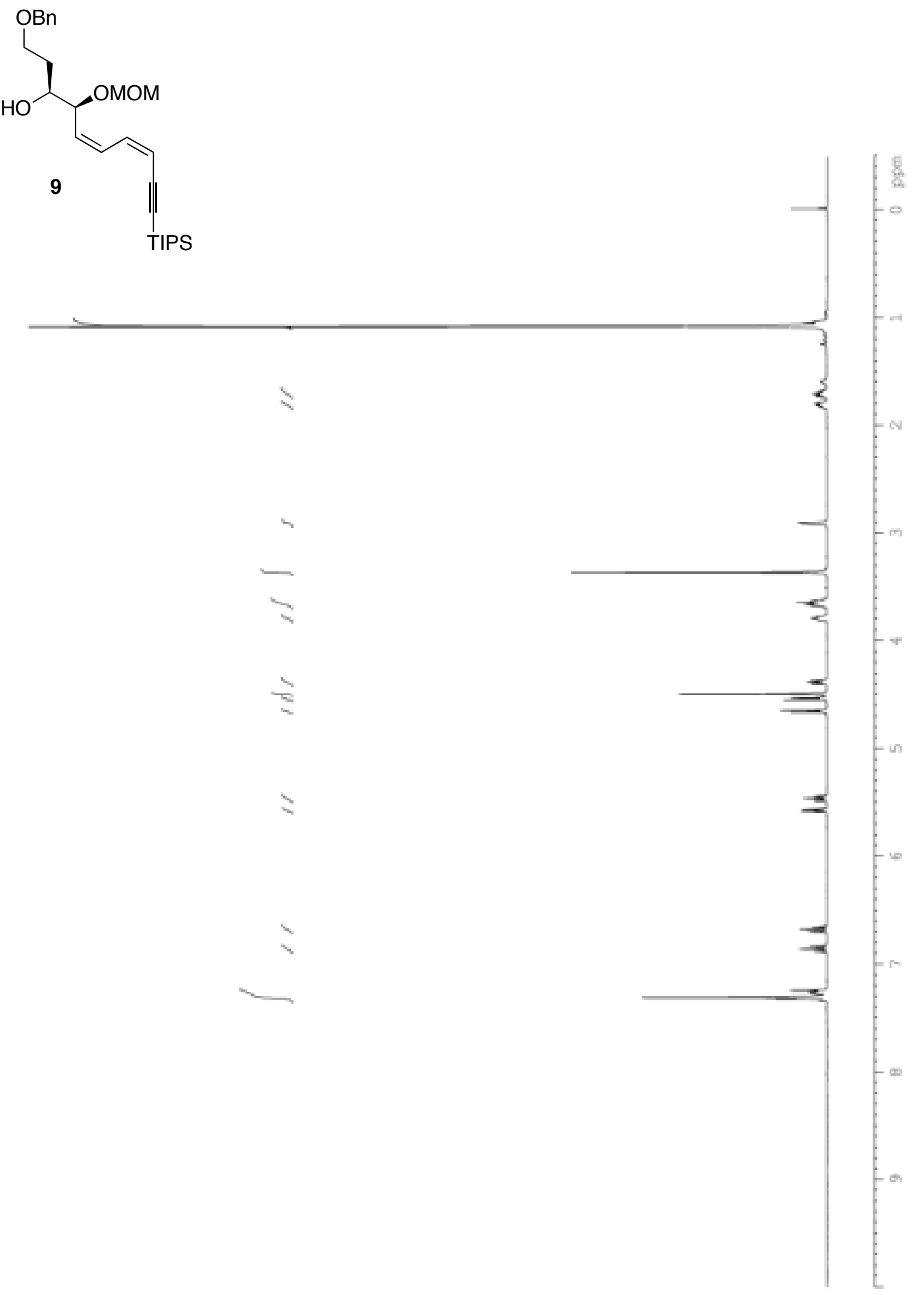

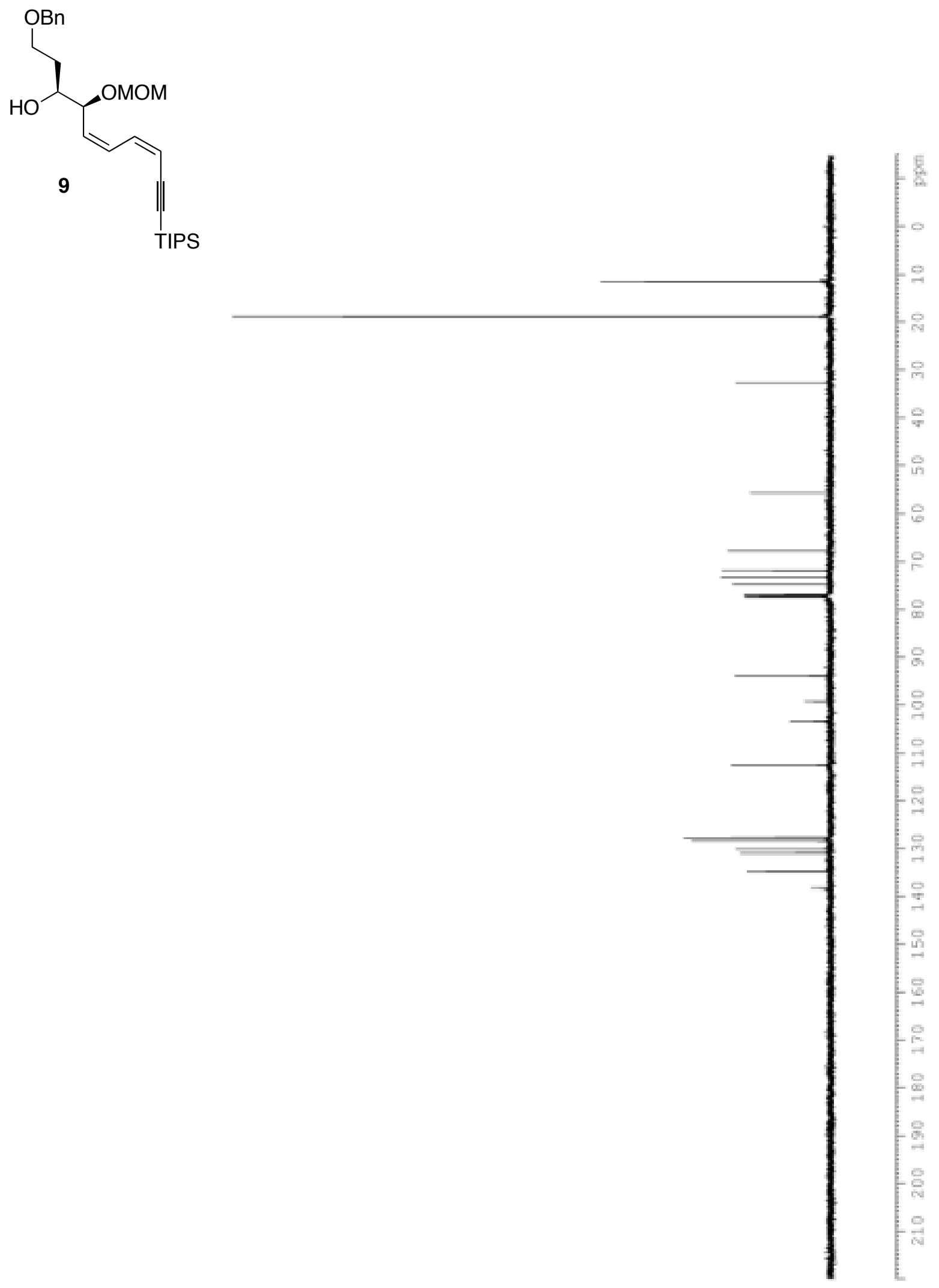

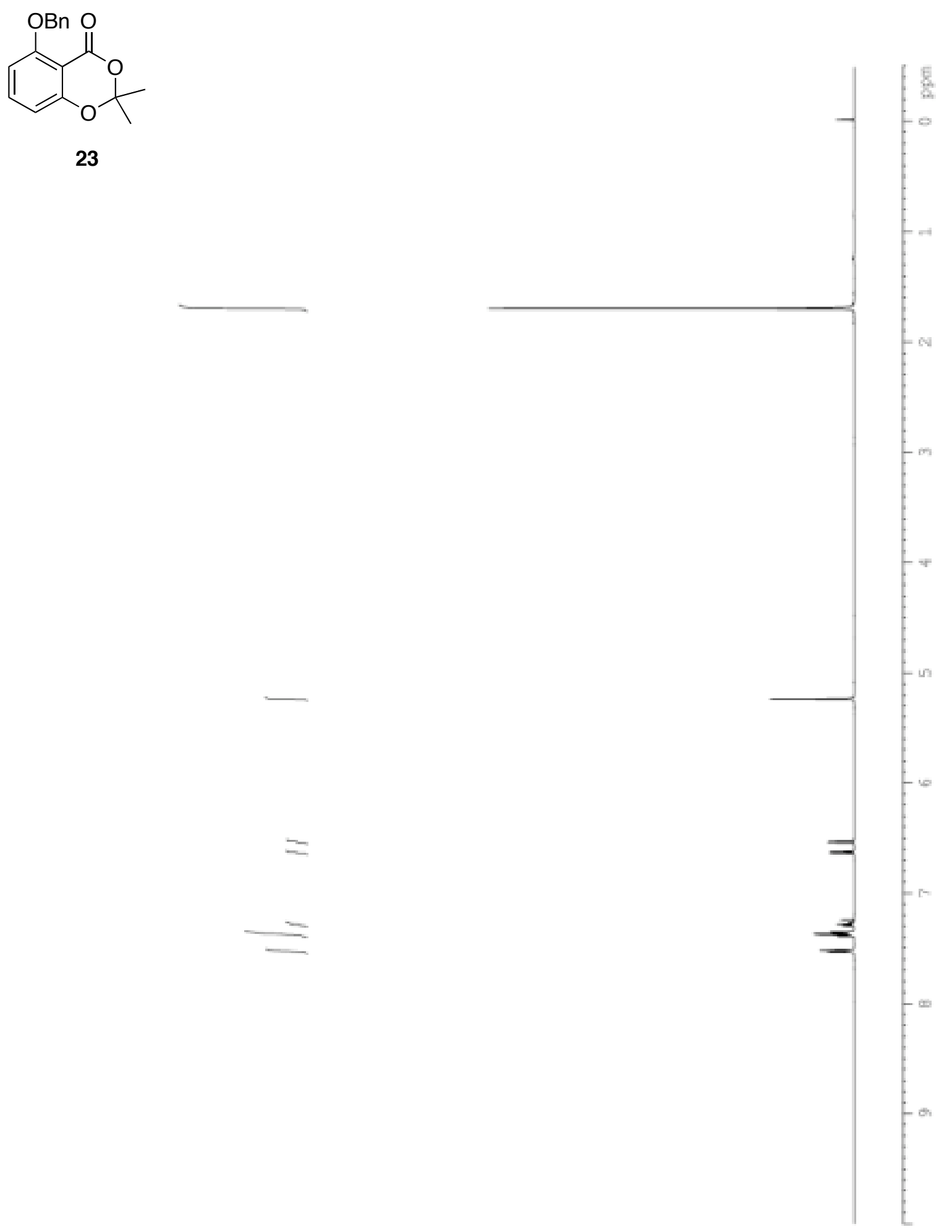


$$
\exists
$$



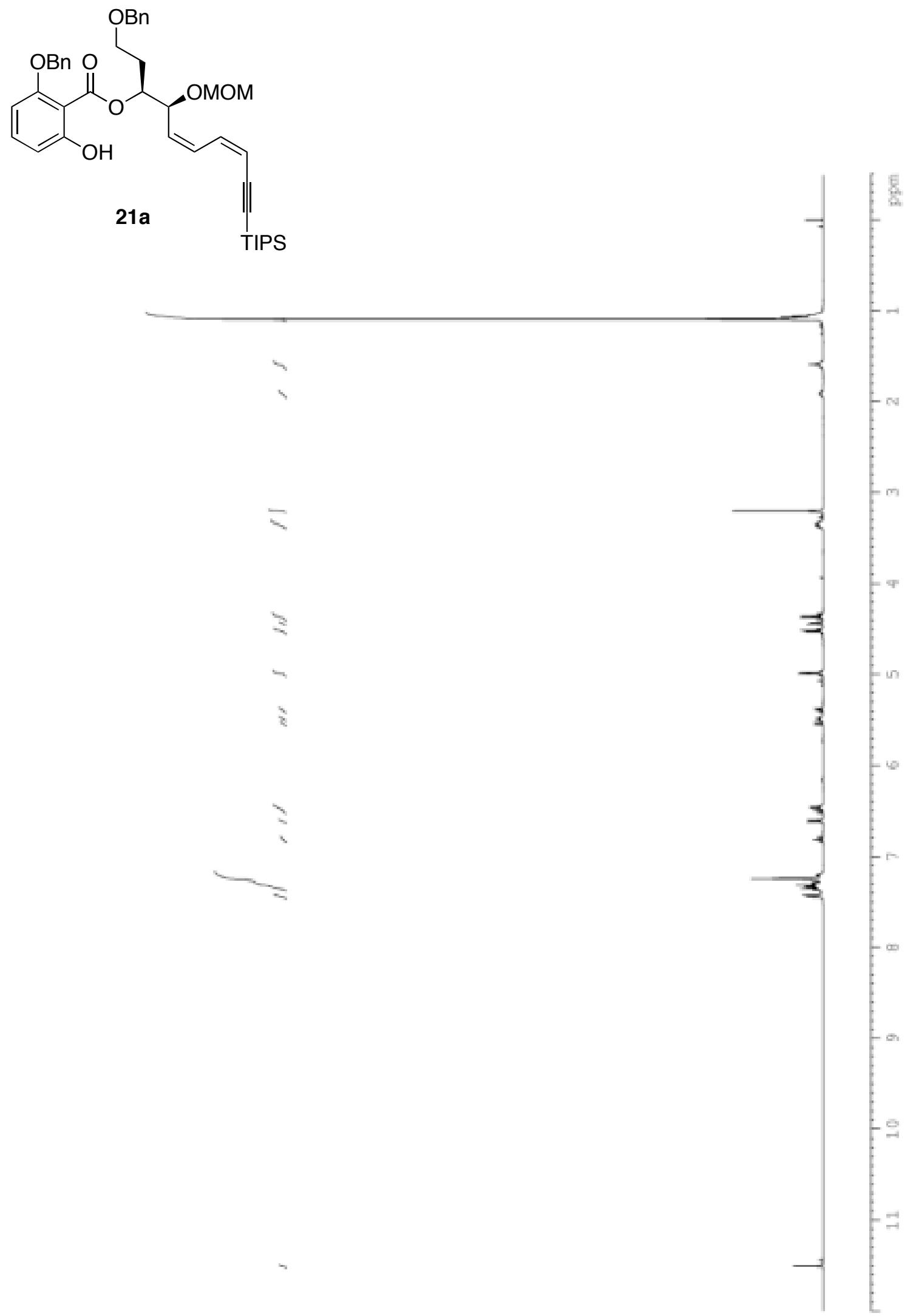

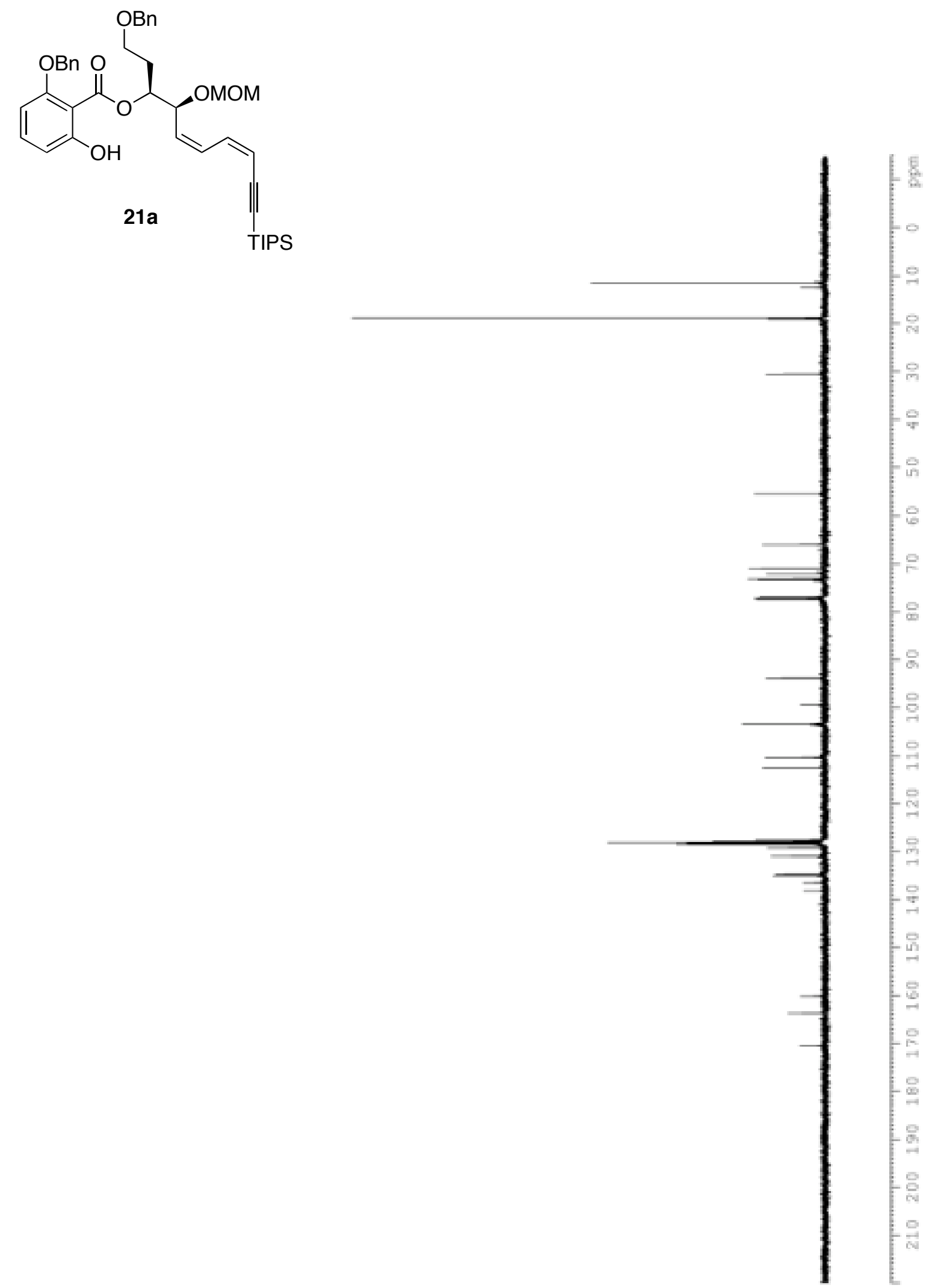

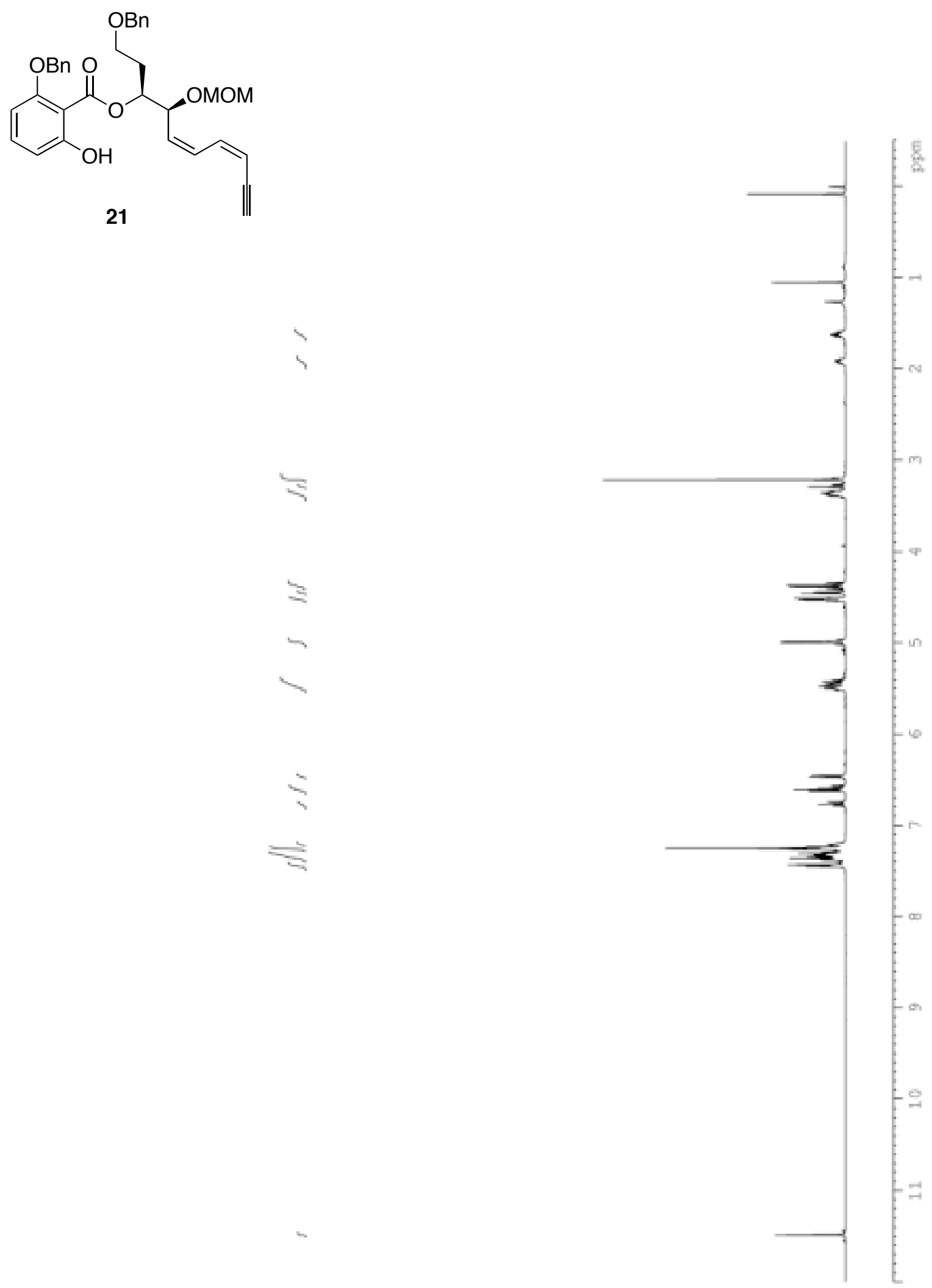

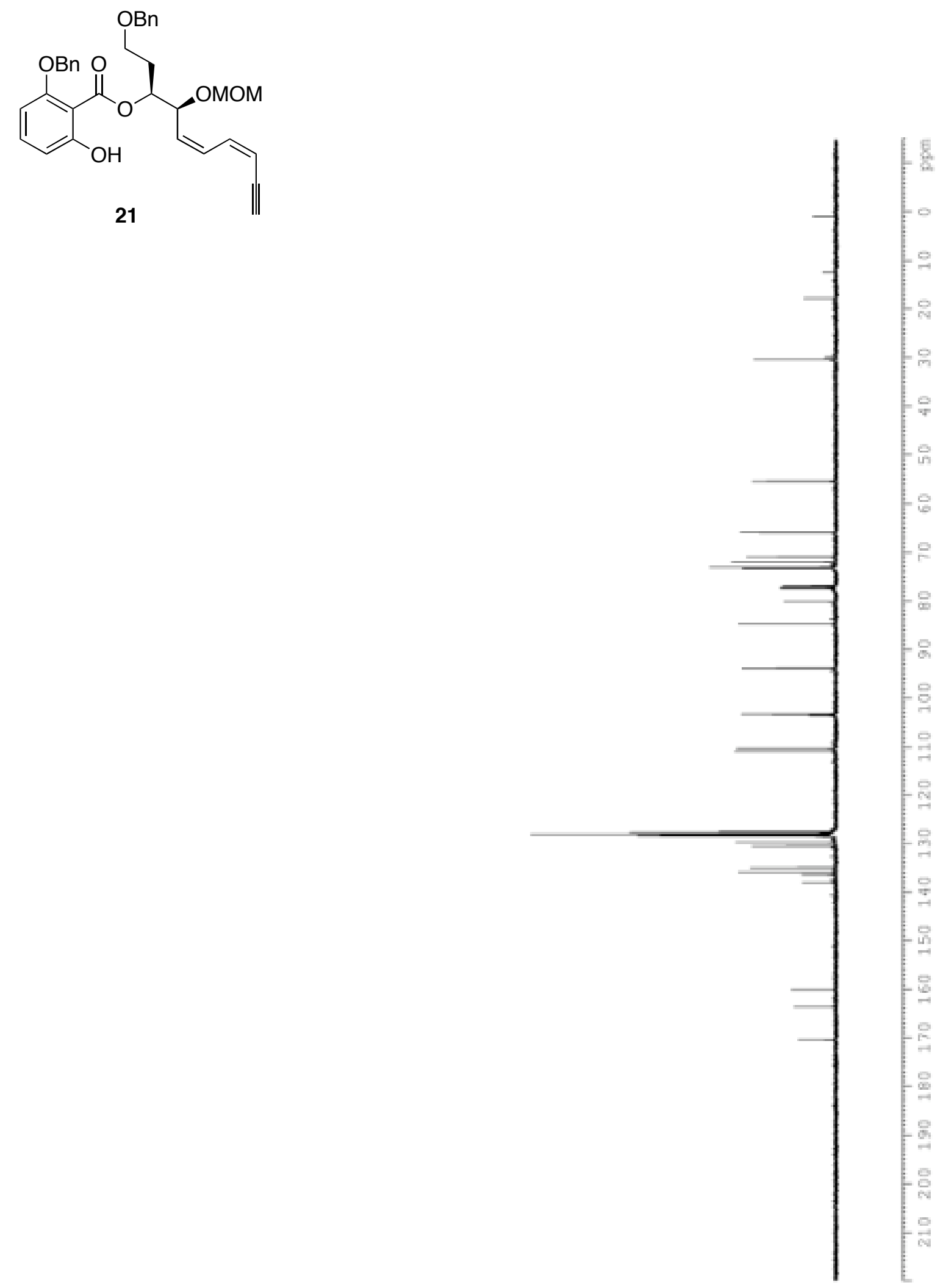


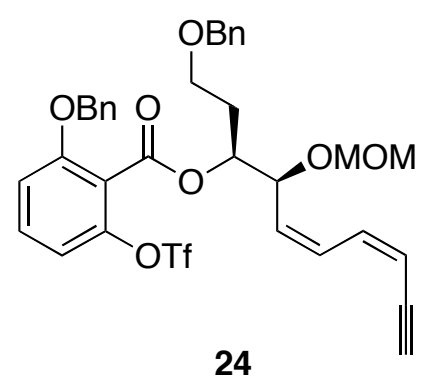

24

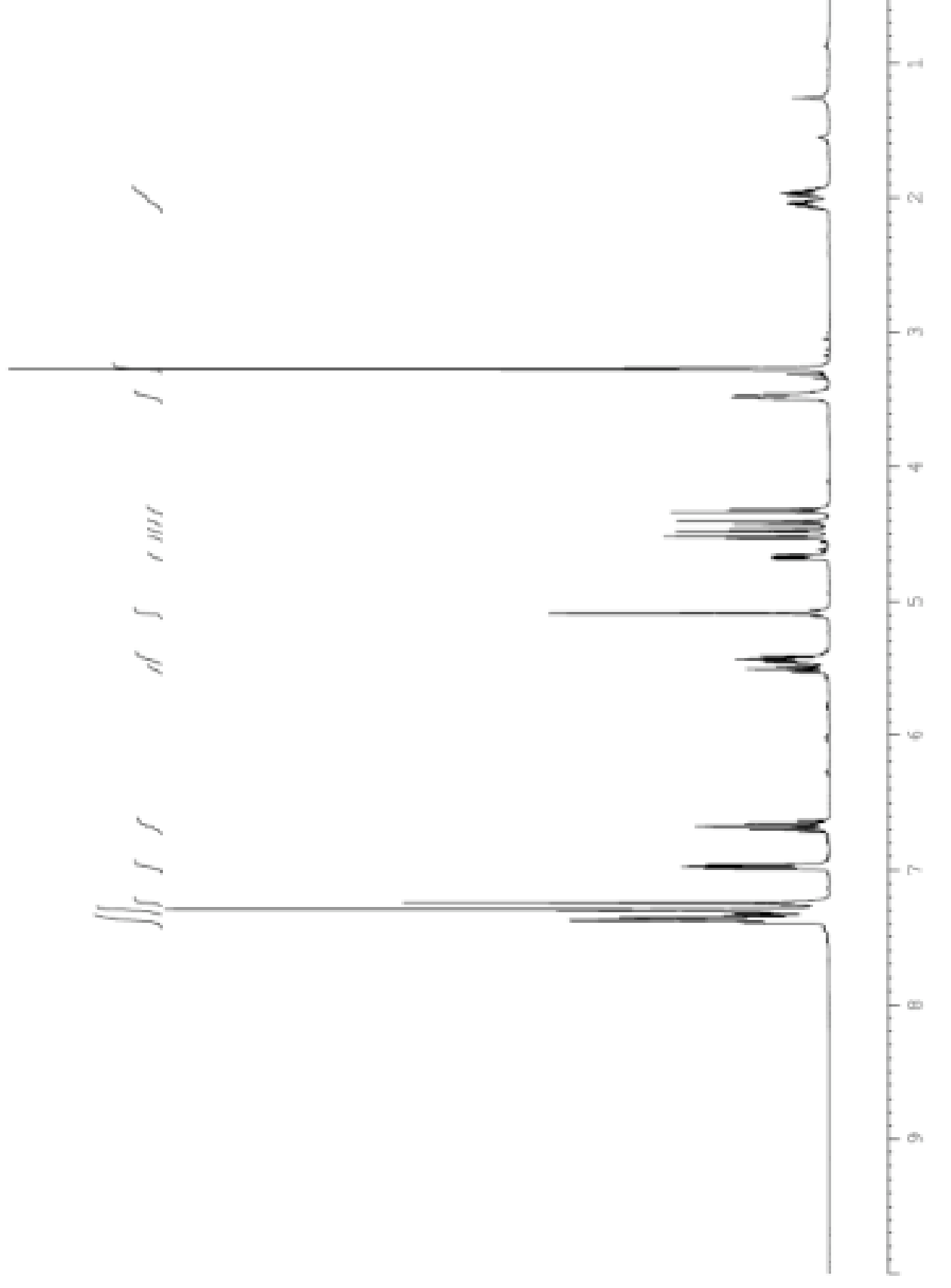



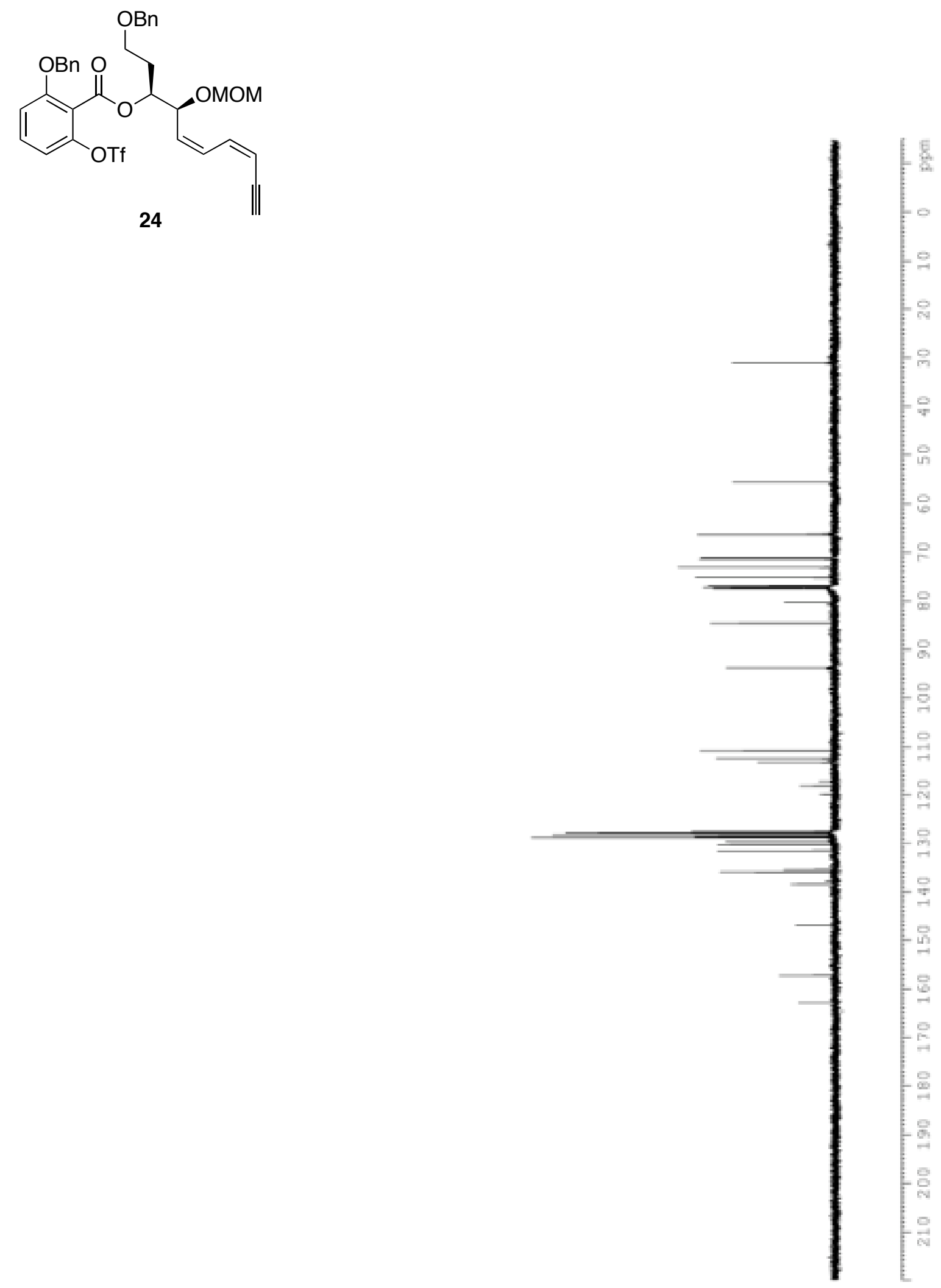


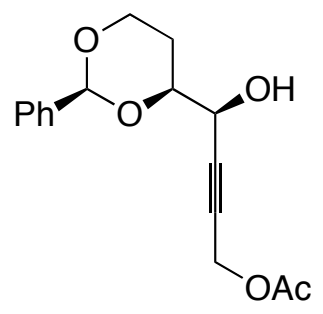

33

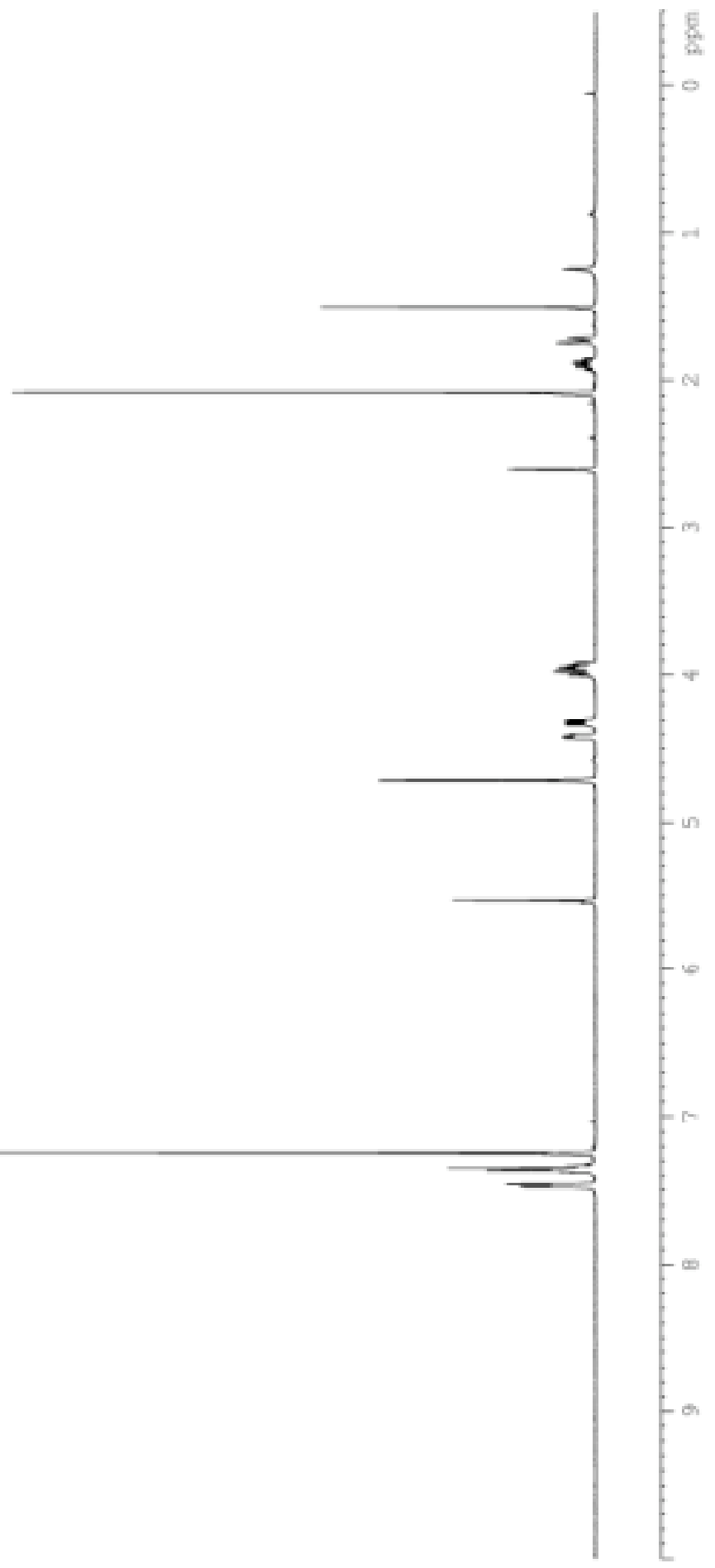

S34 

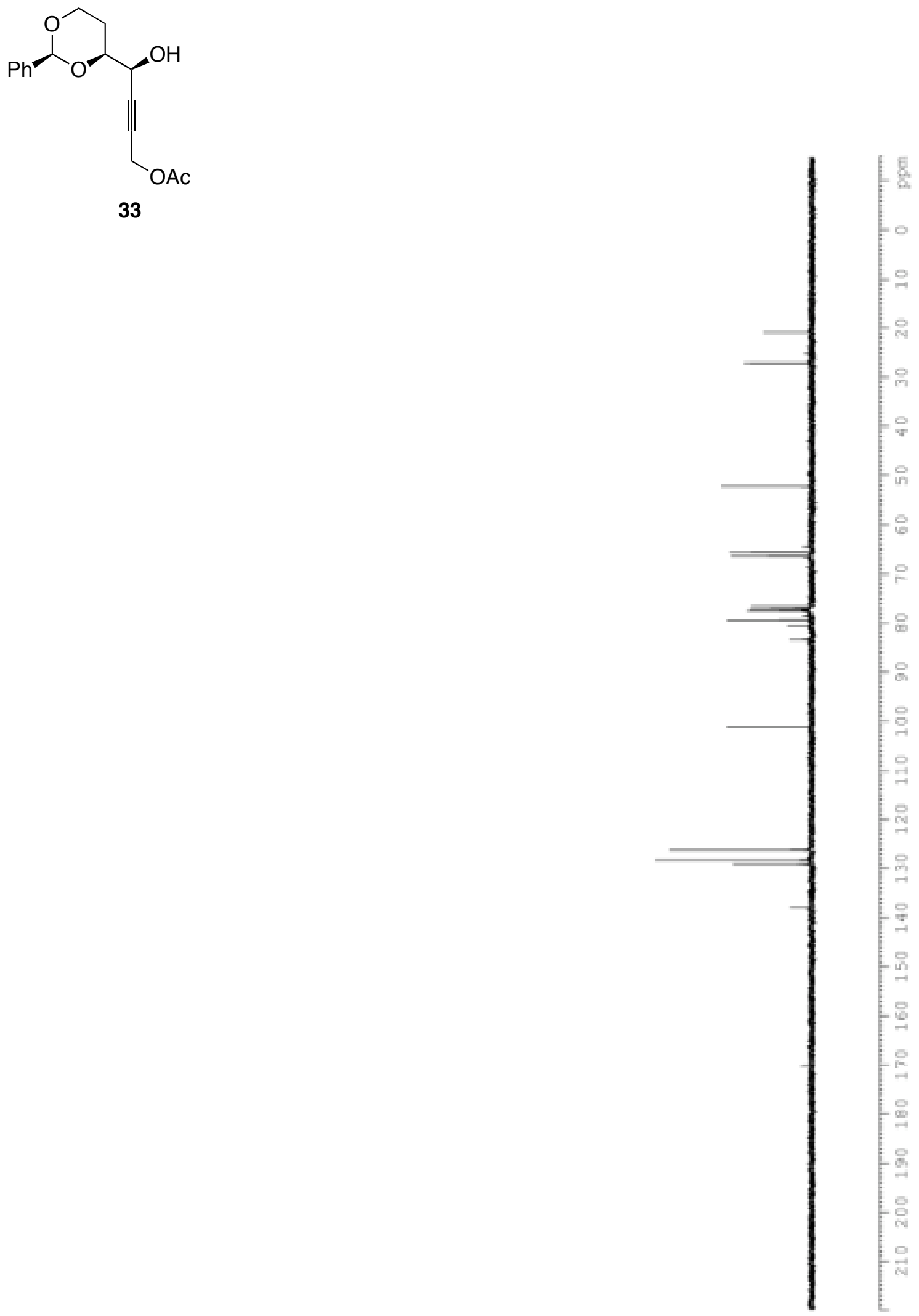


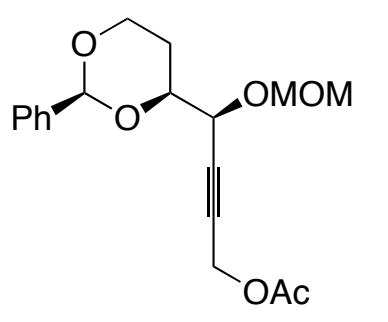

34

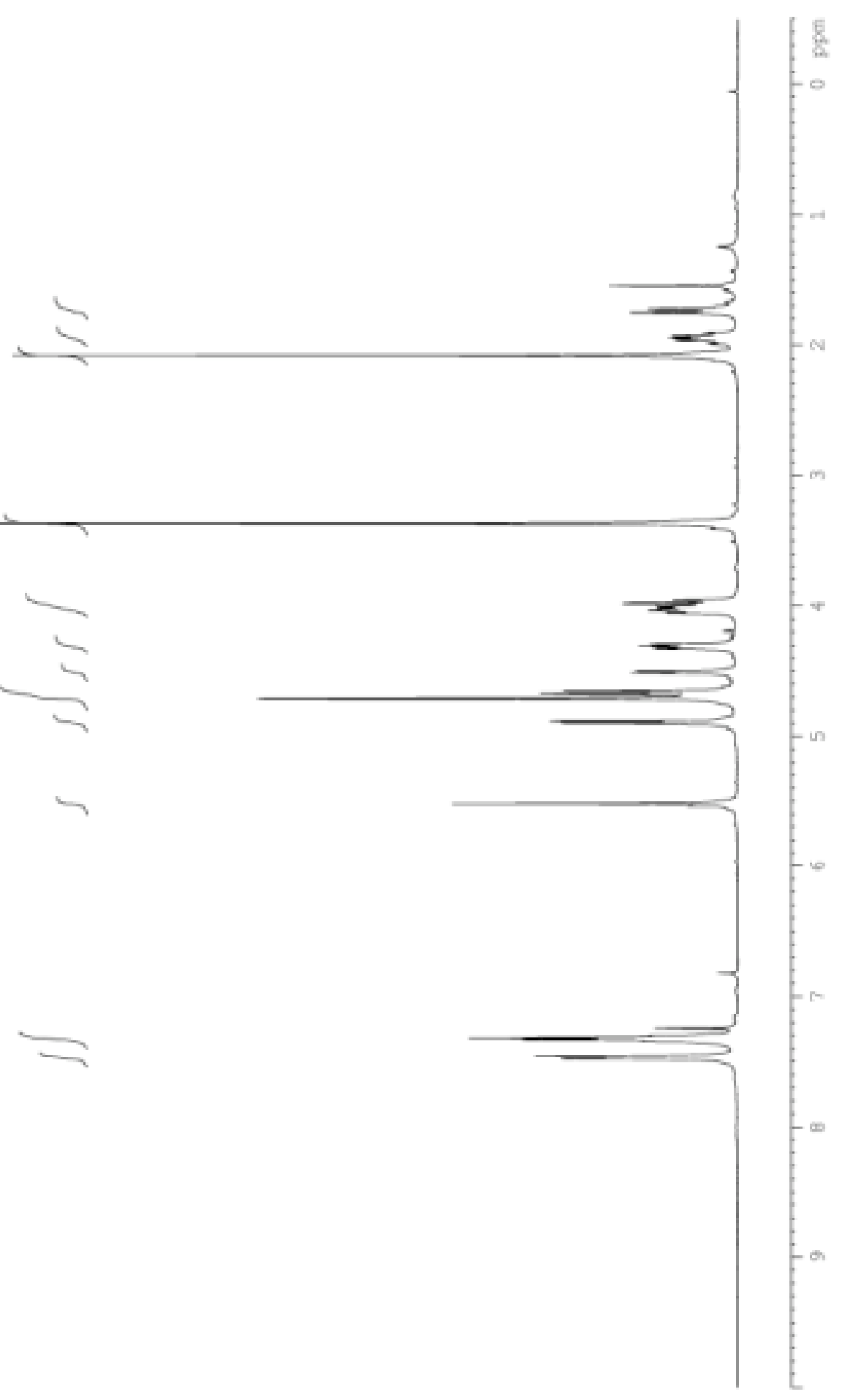

S36 


$$
1
$$




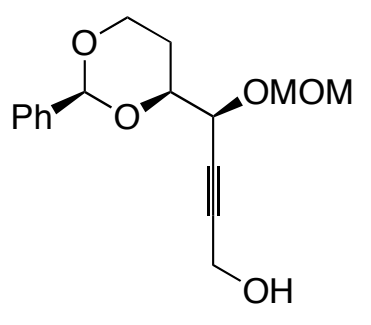

35
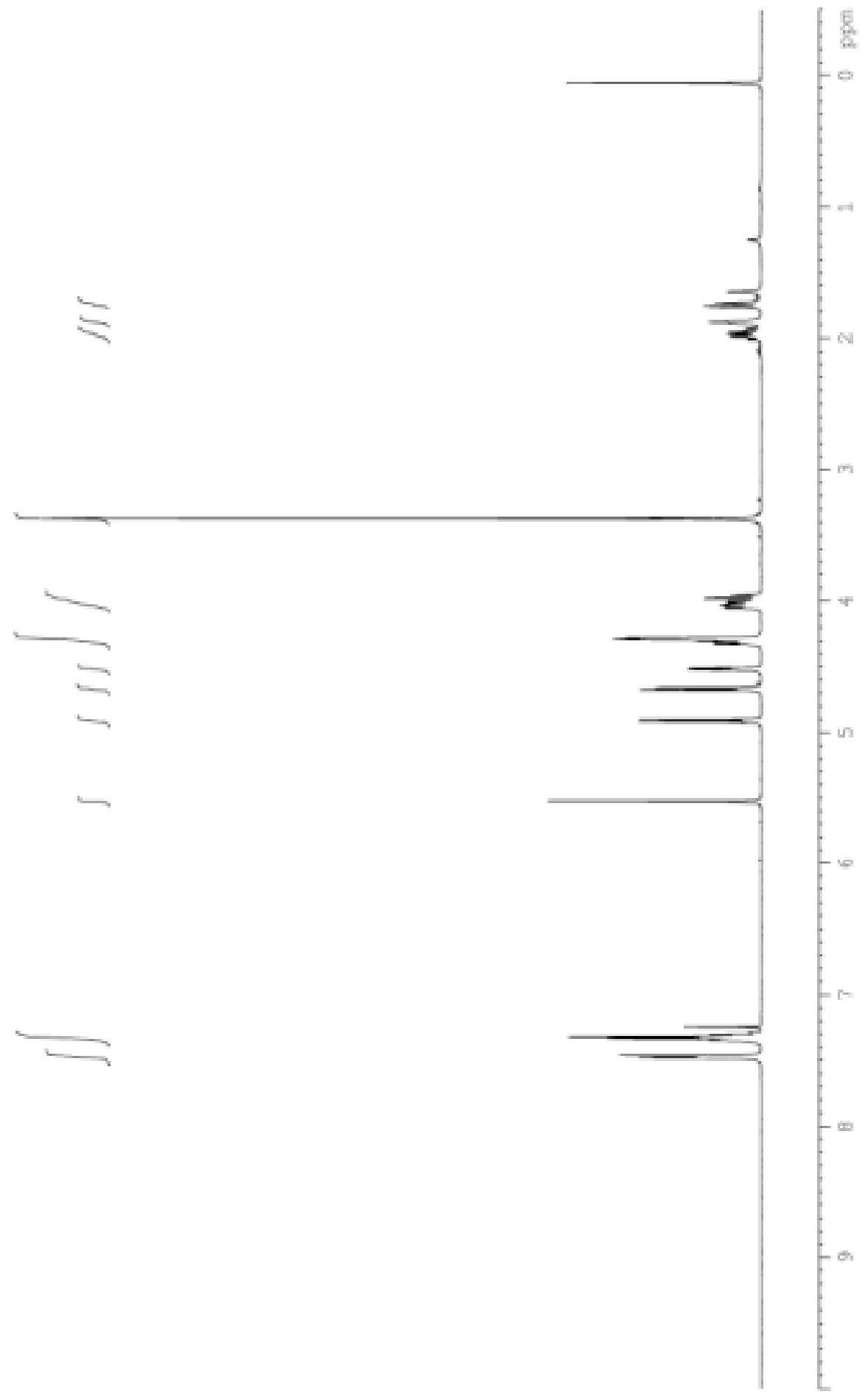


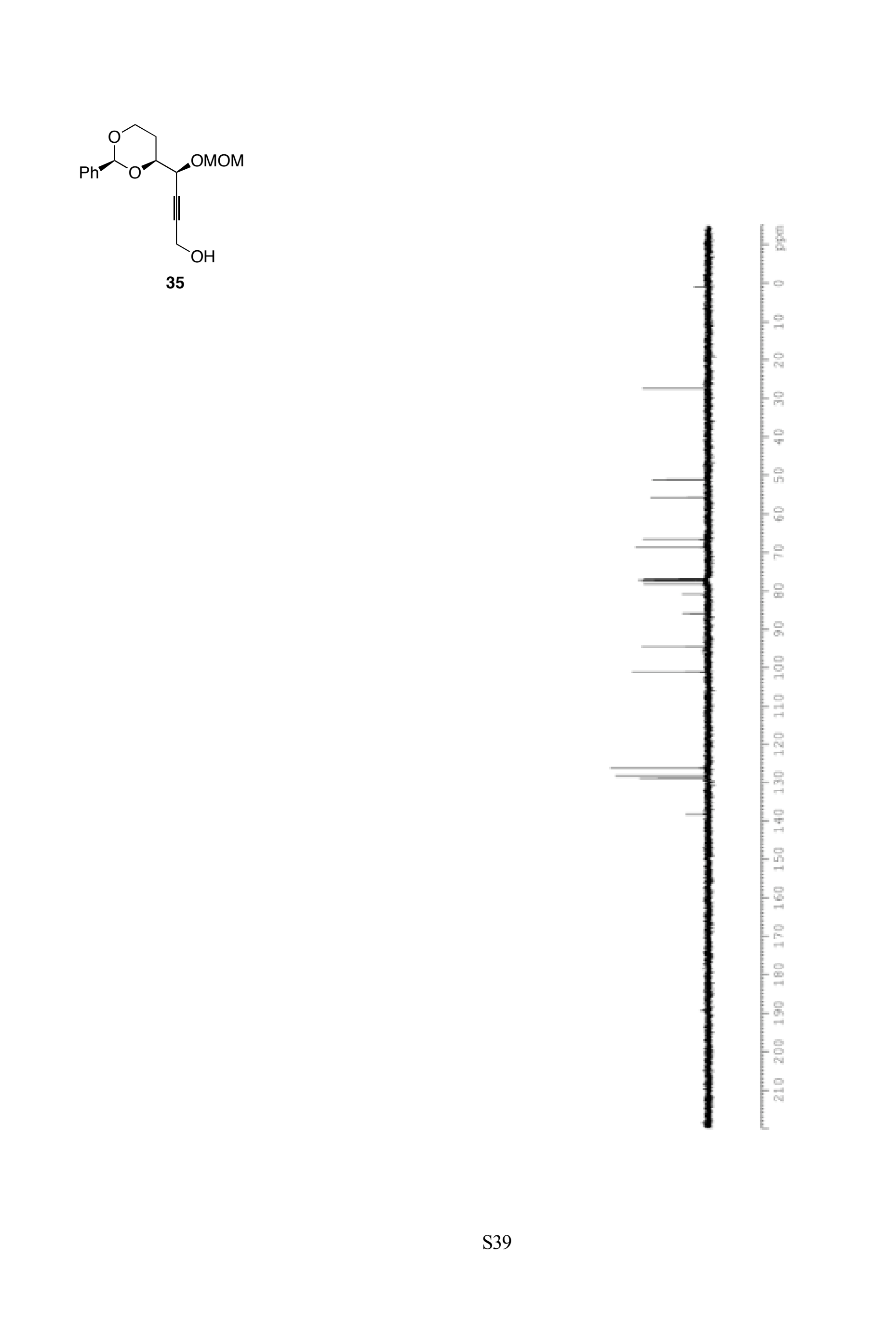




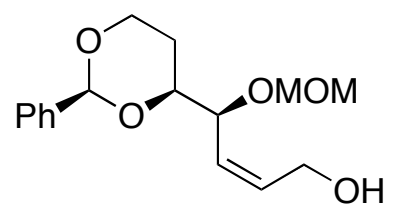

36

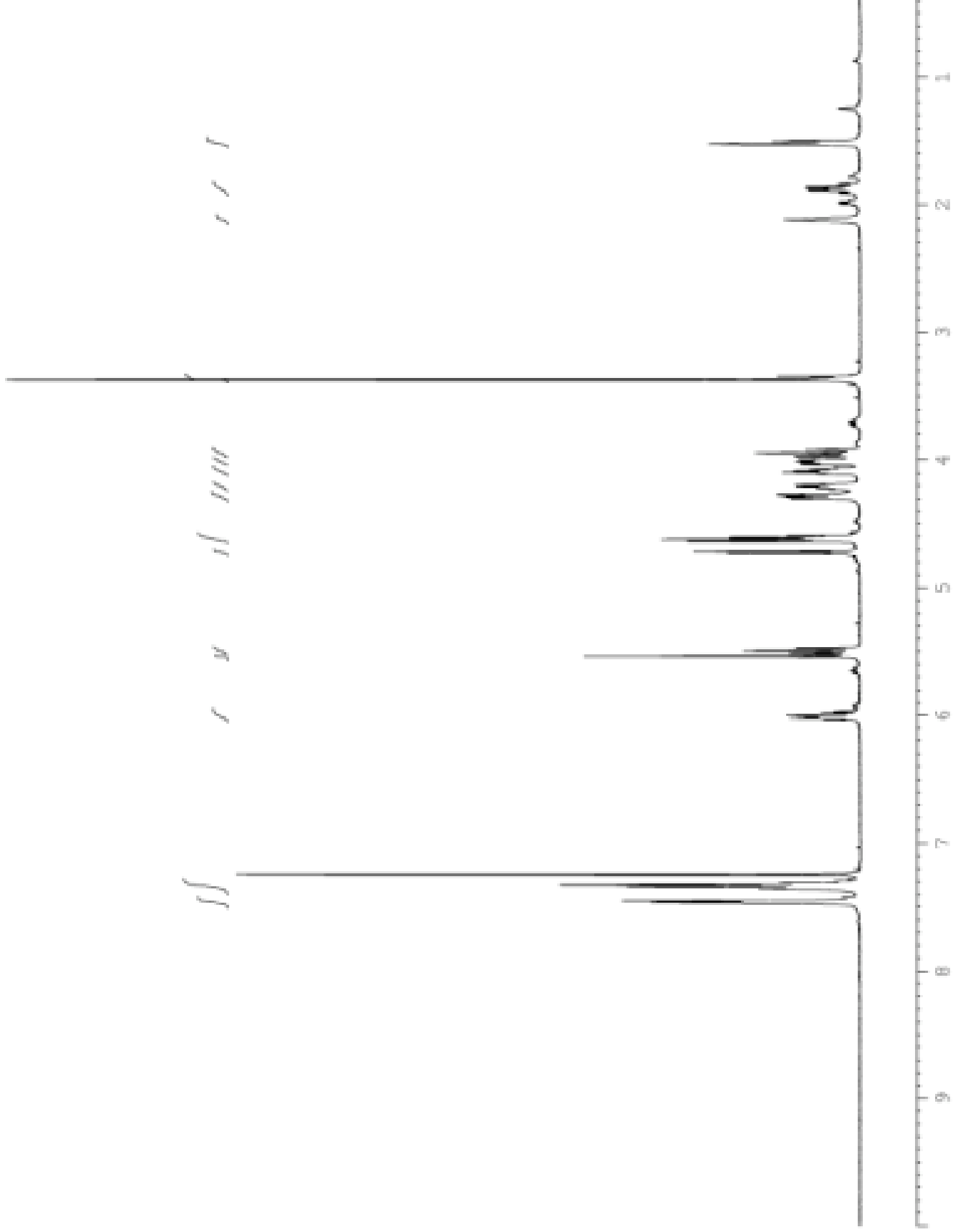




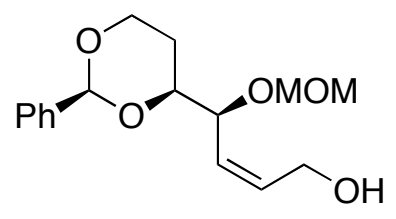

36

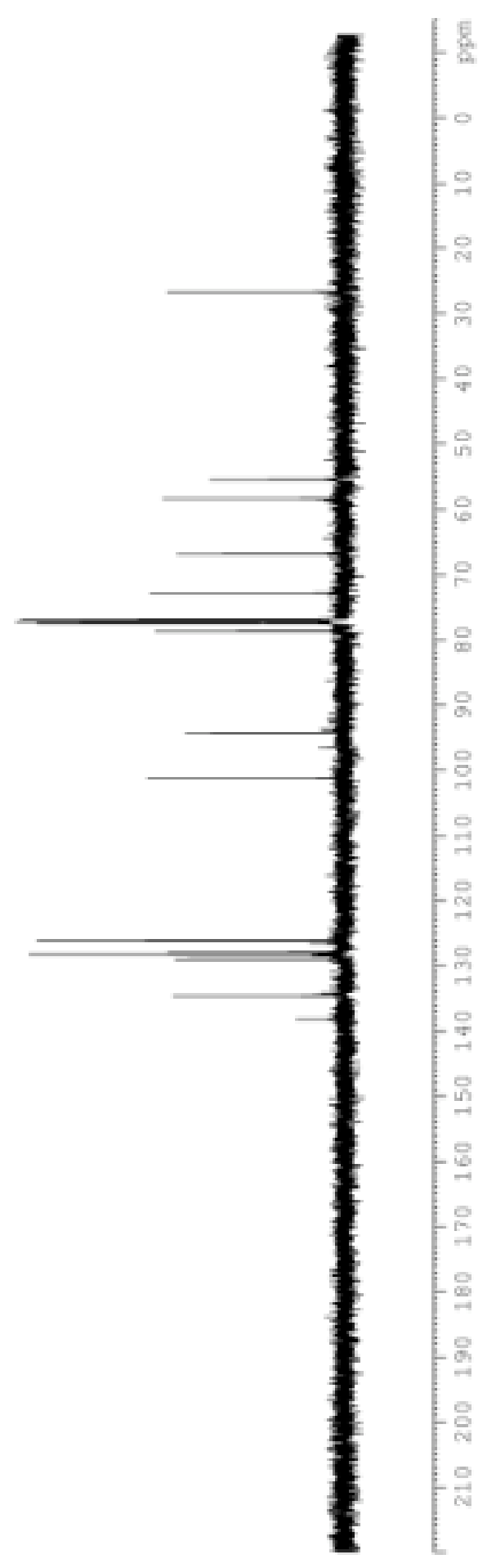




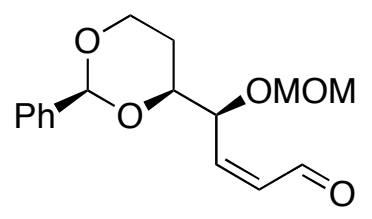

31

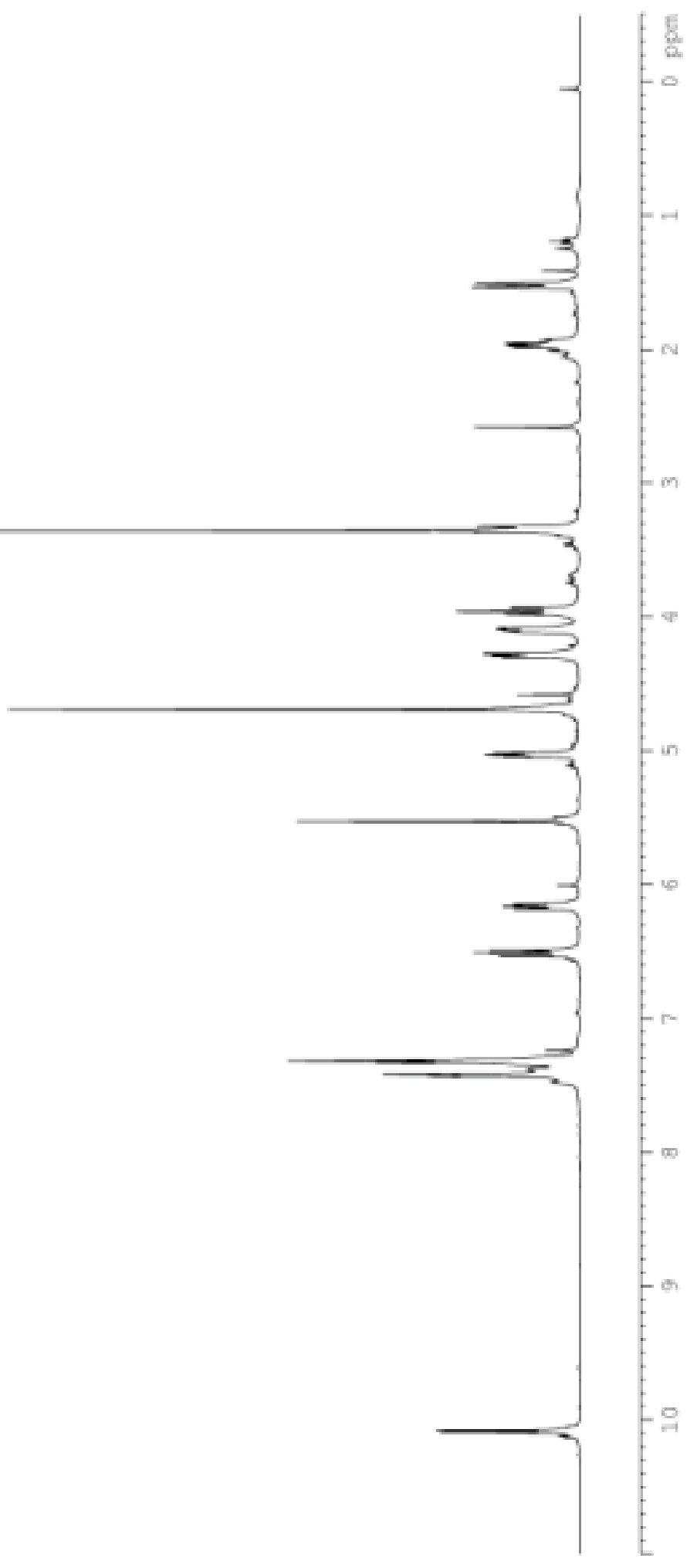




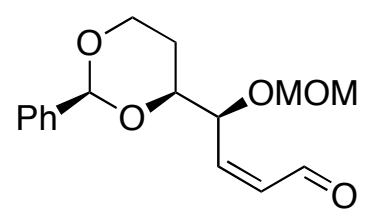

31

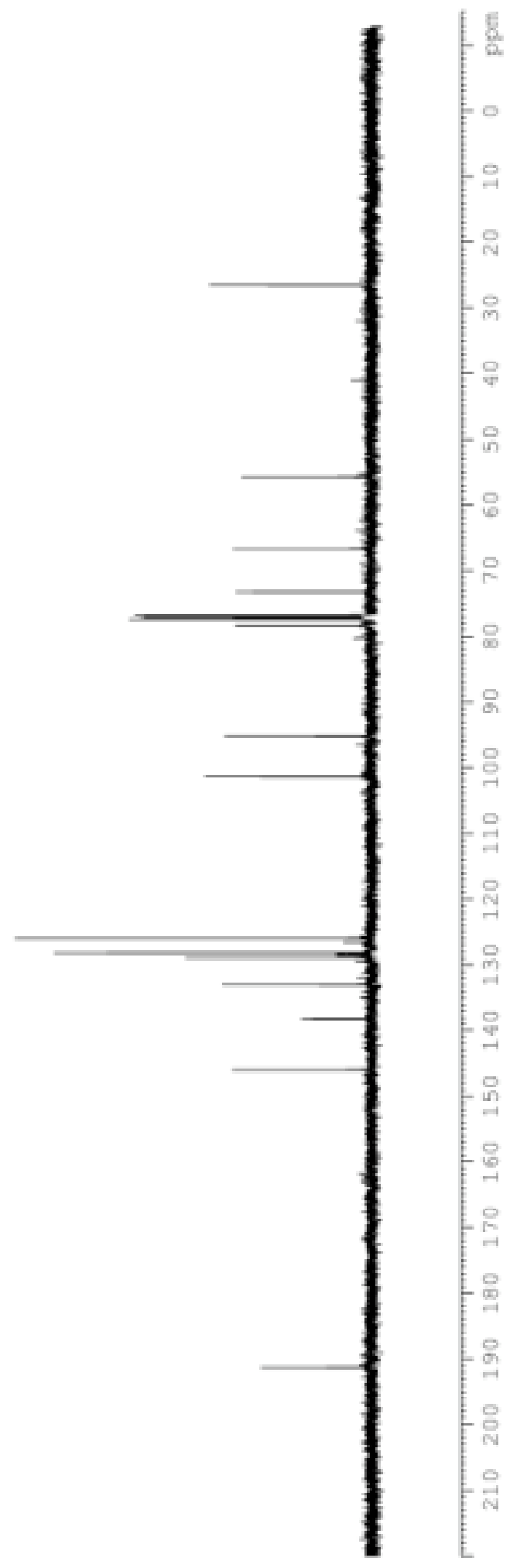



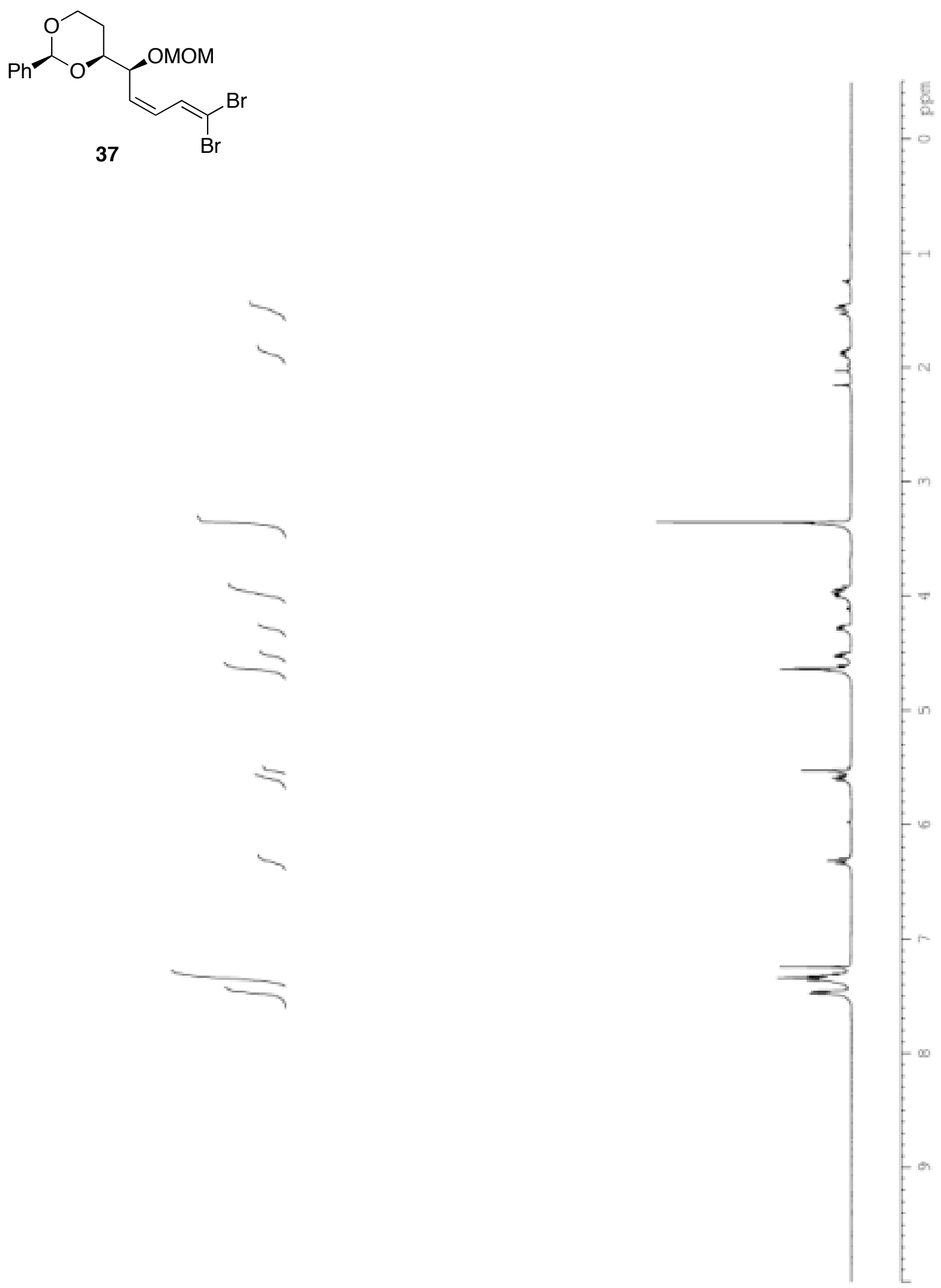
<smiles>CO[C@H](/C=C\C=C(Br)Br)[C@H]1CCOC(c2ccccc2)O1</smiles>

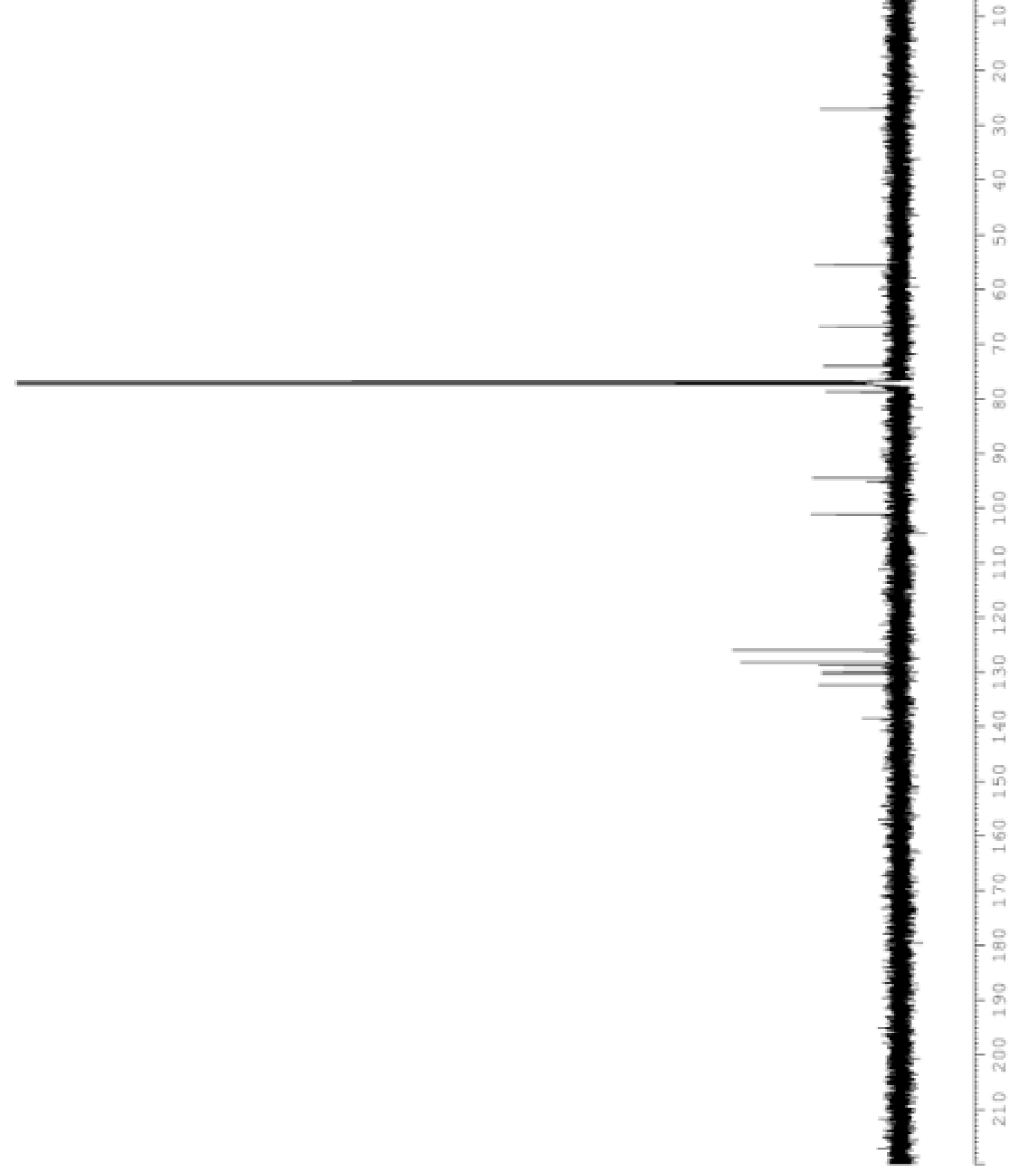



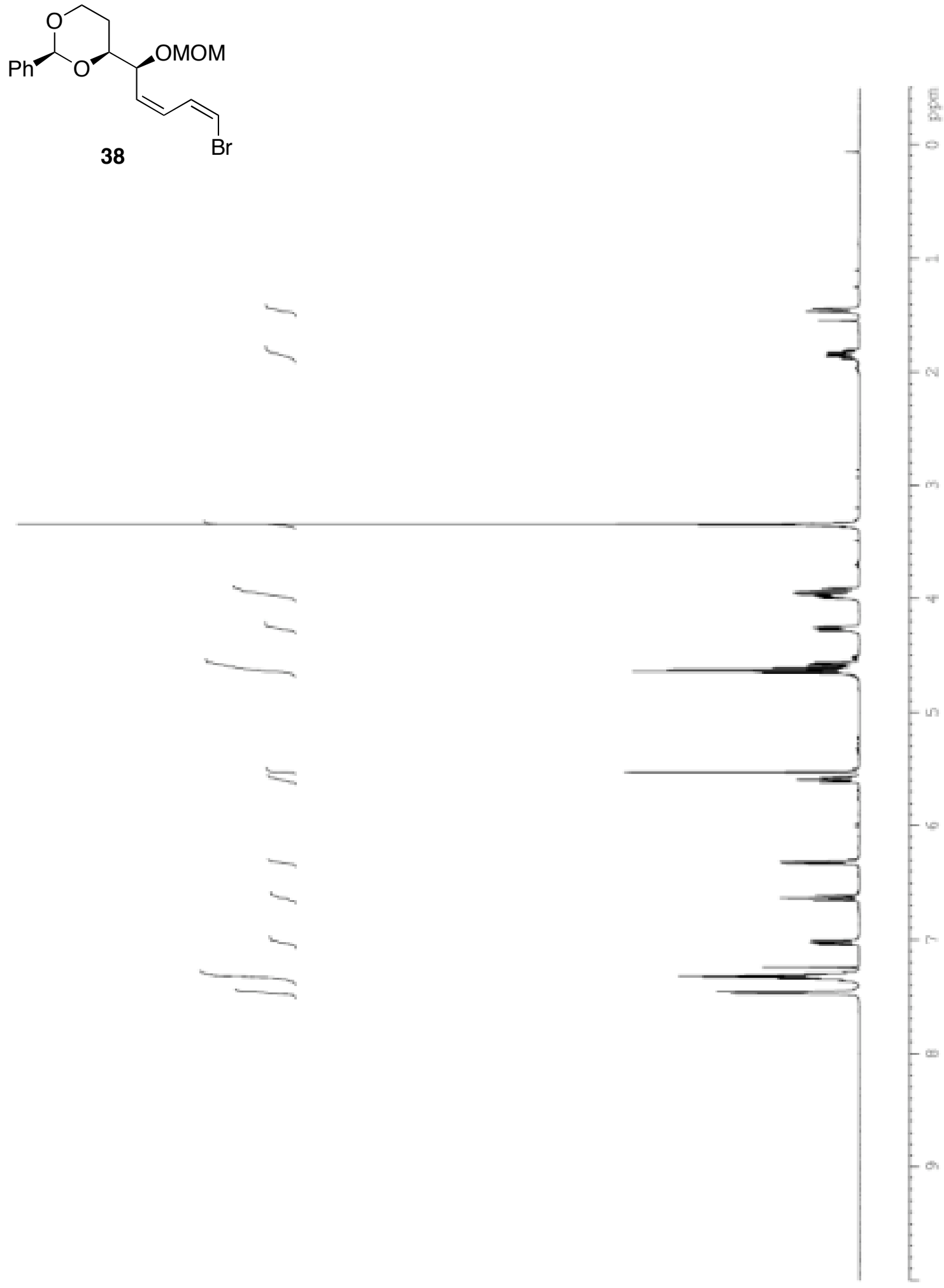

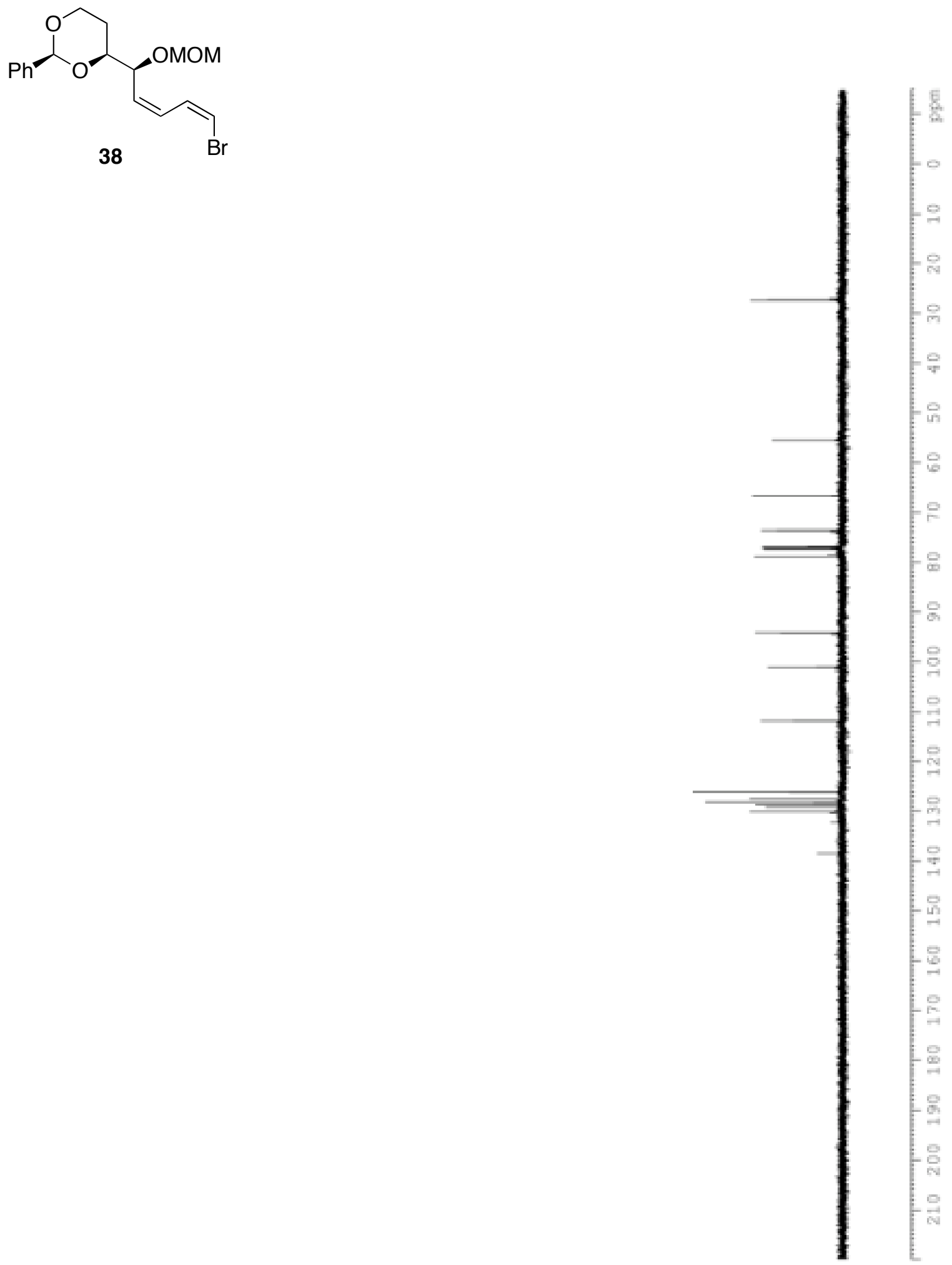

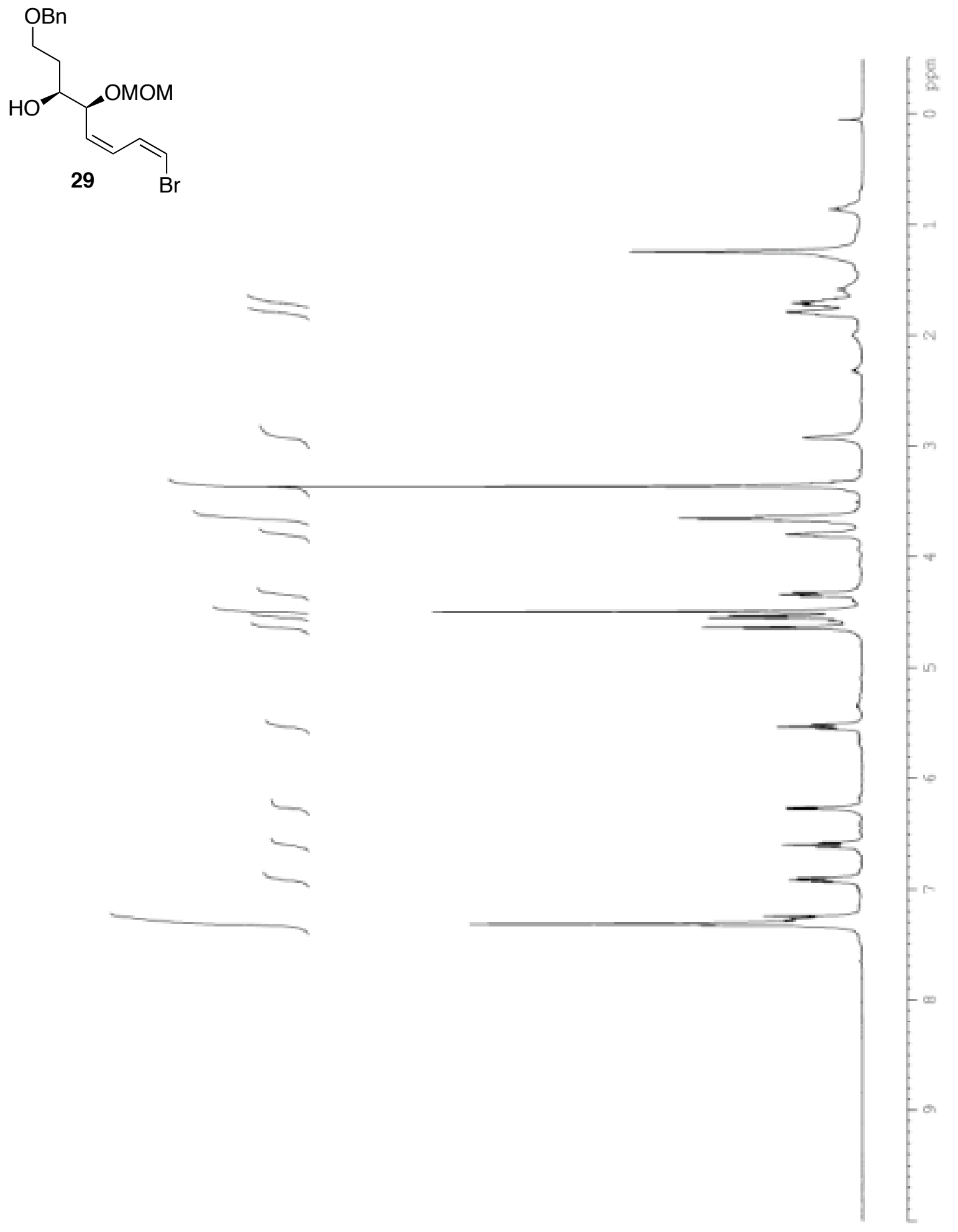

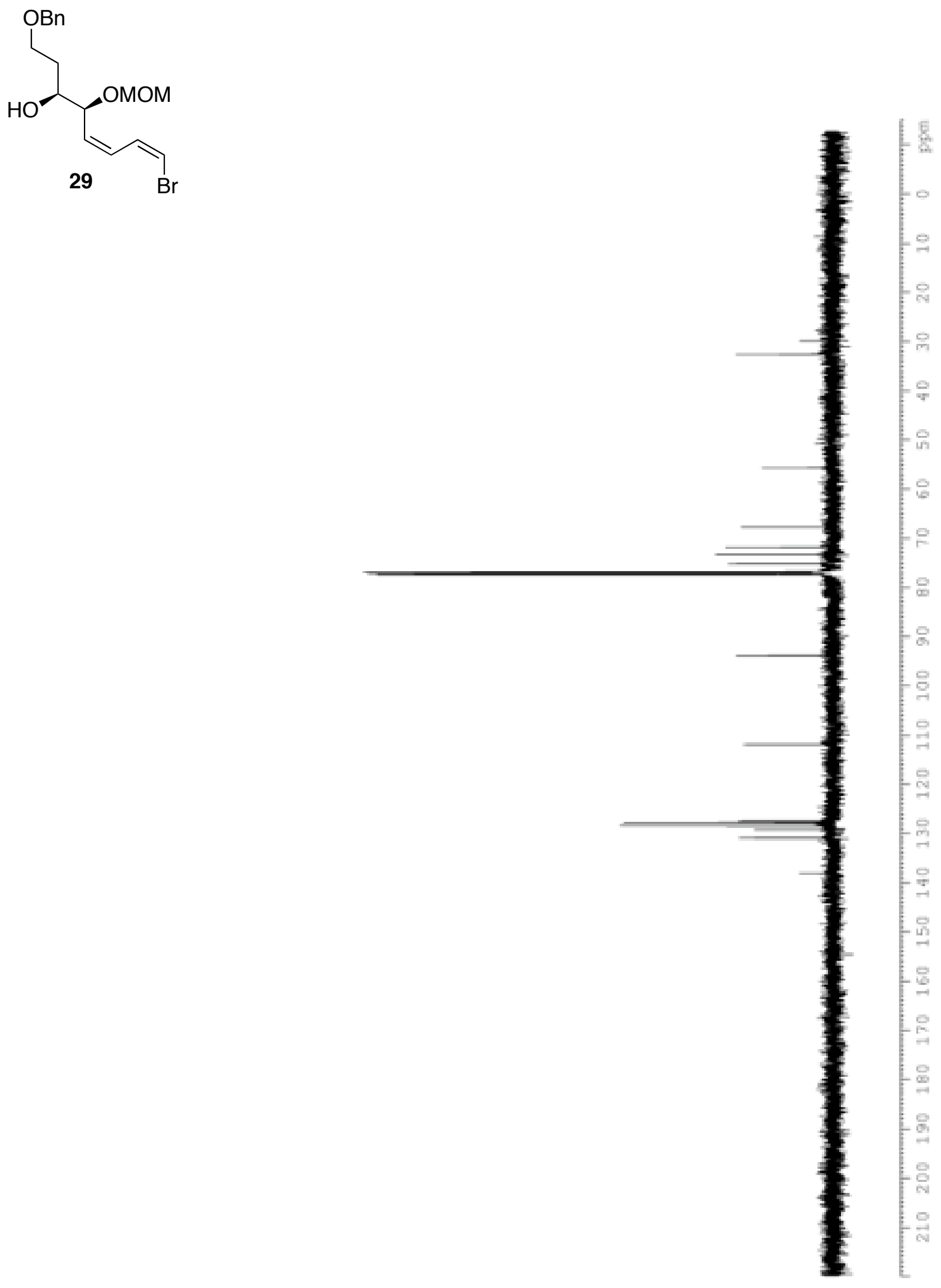

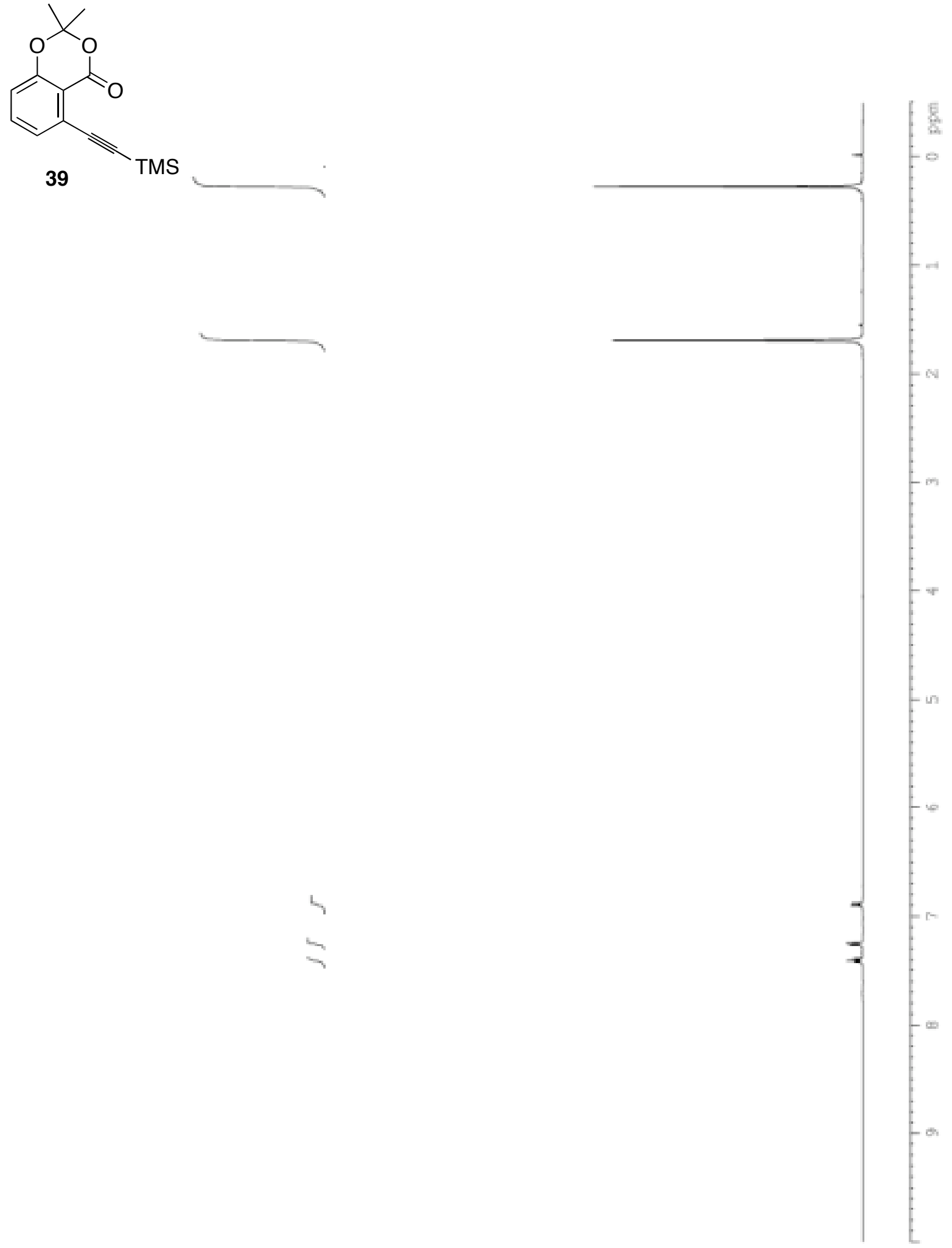

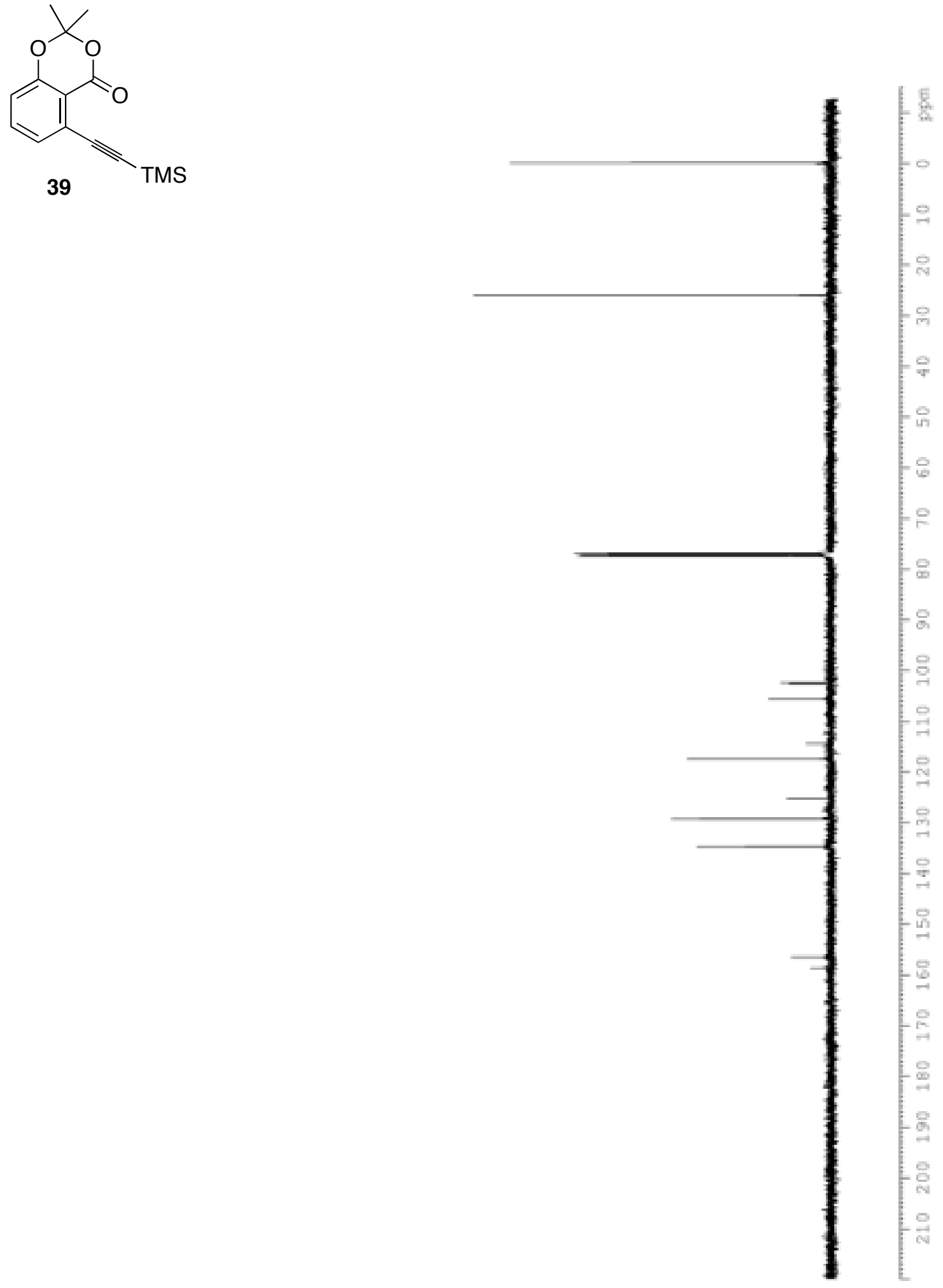

S51 

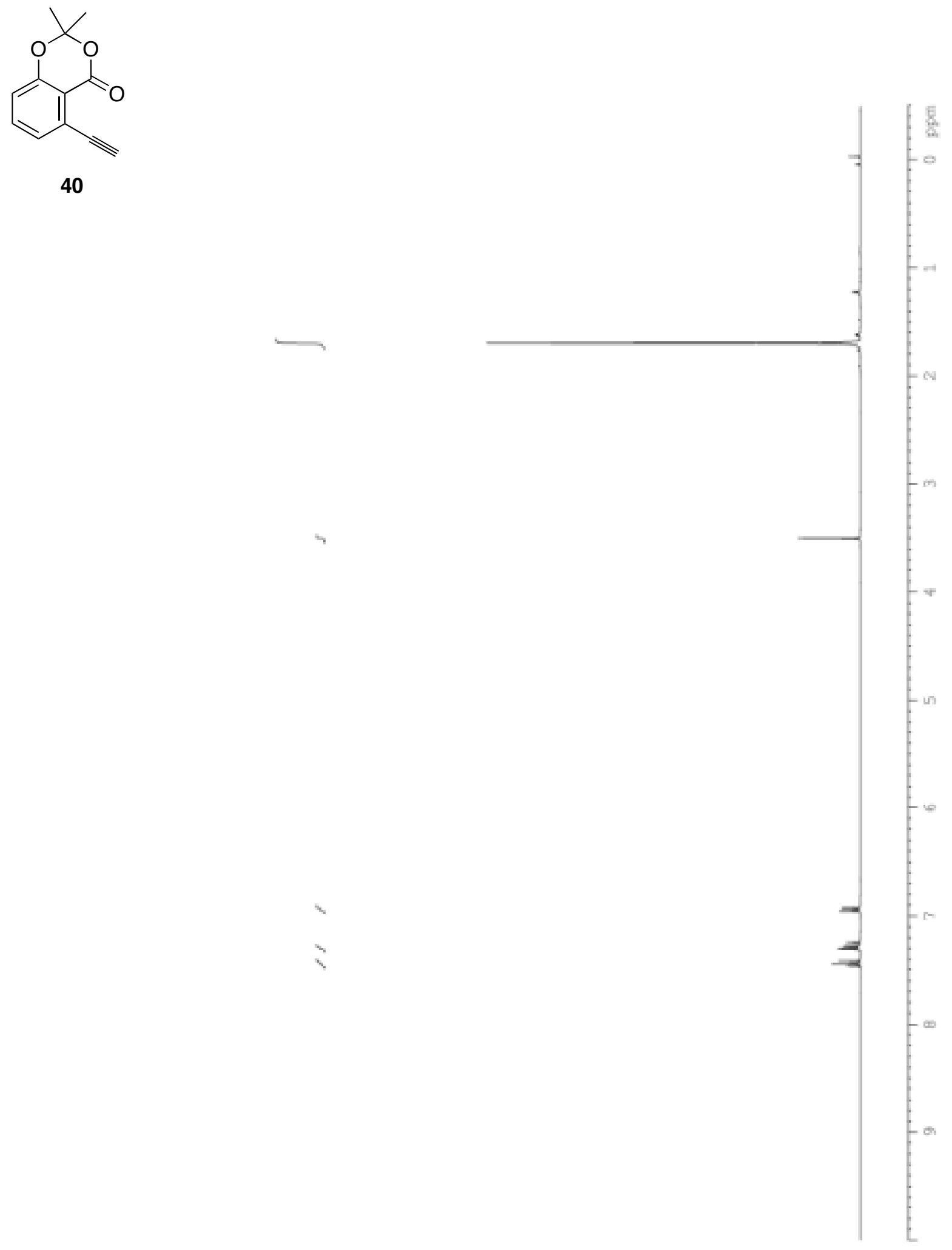

S52 

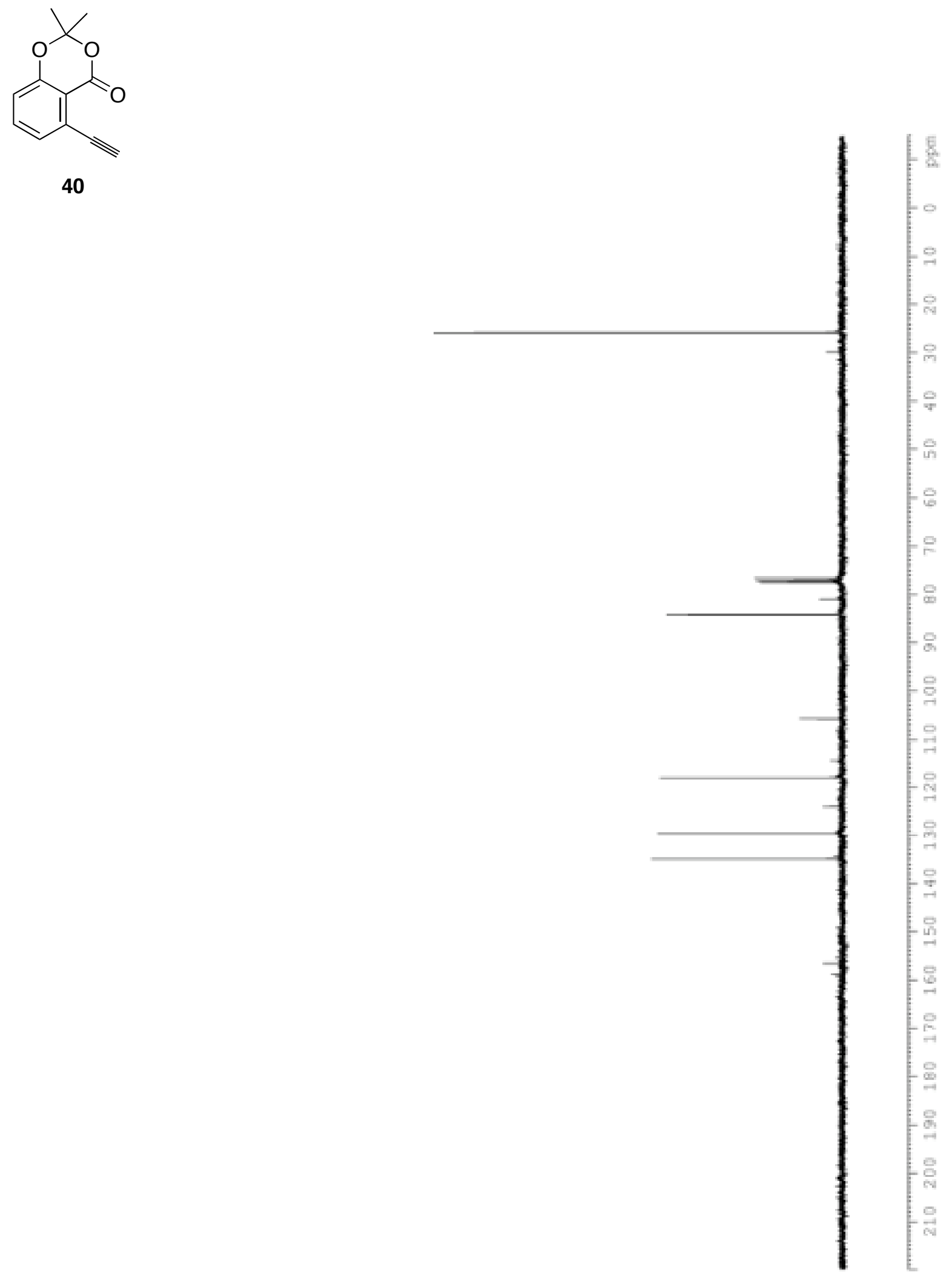

S53 

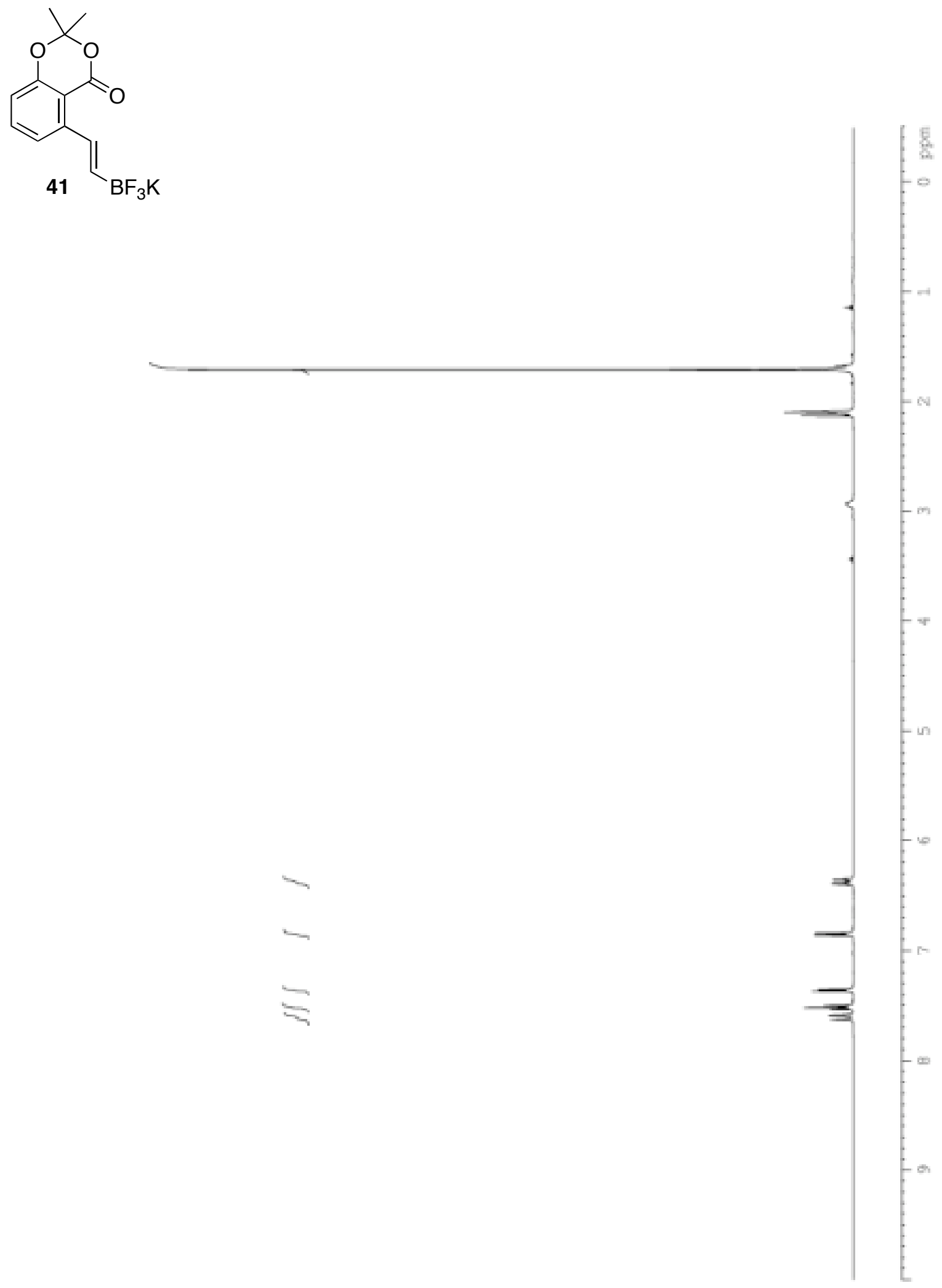

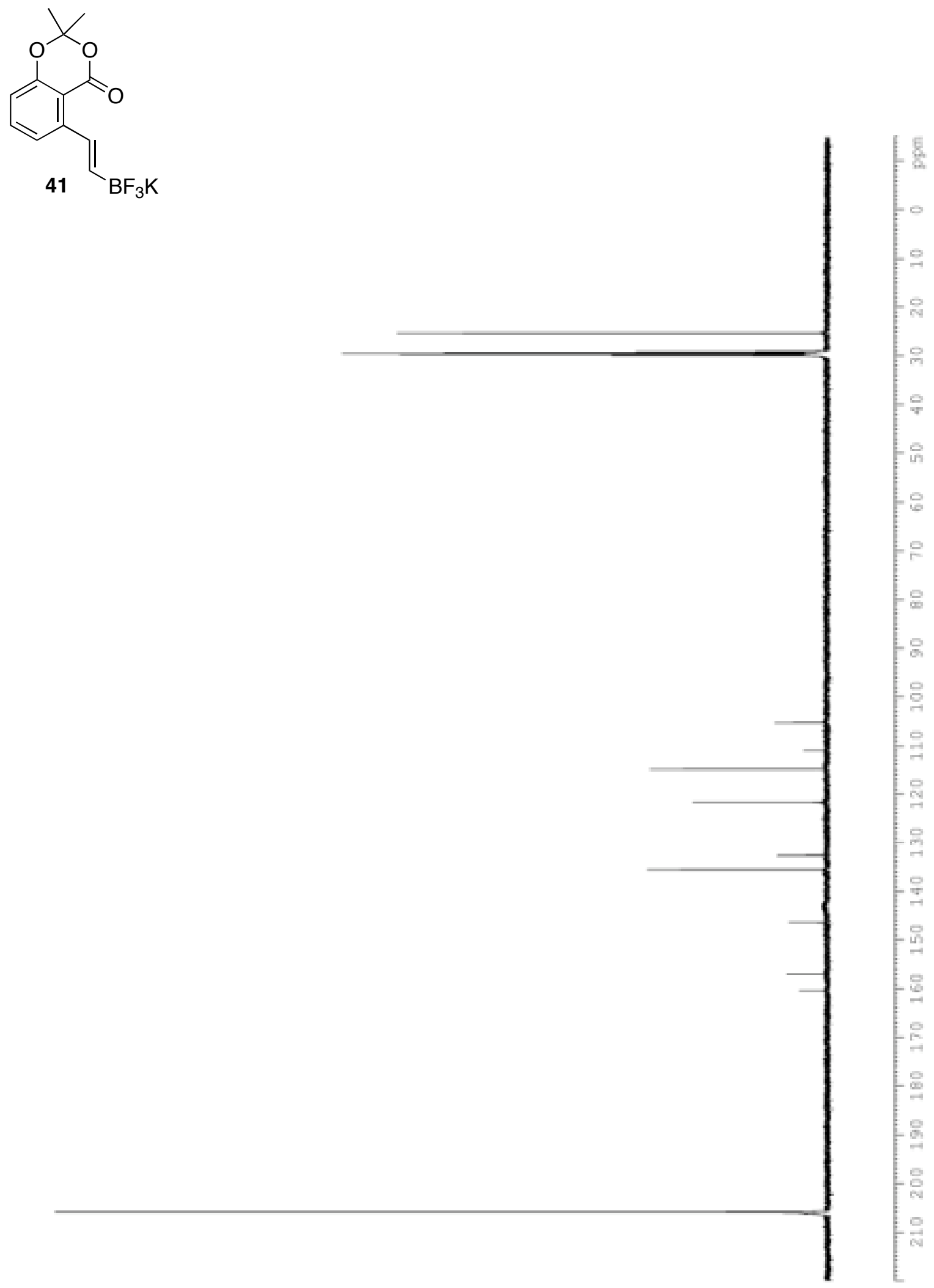


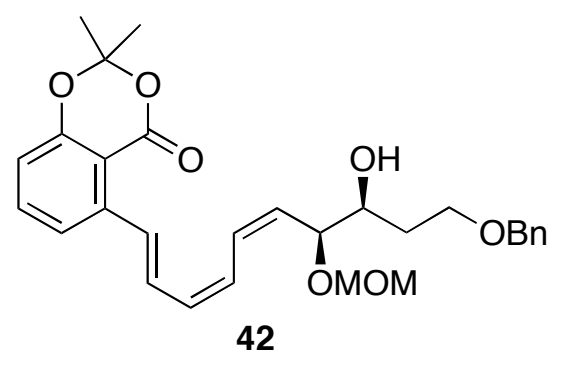

42

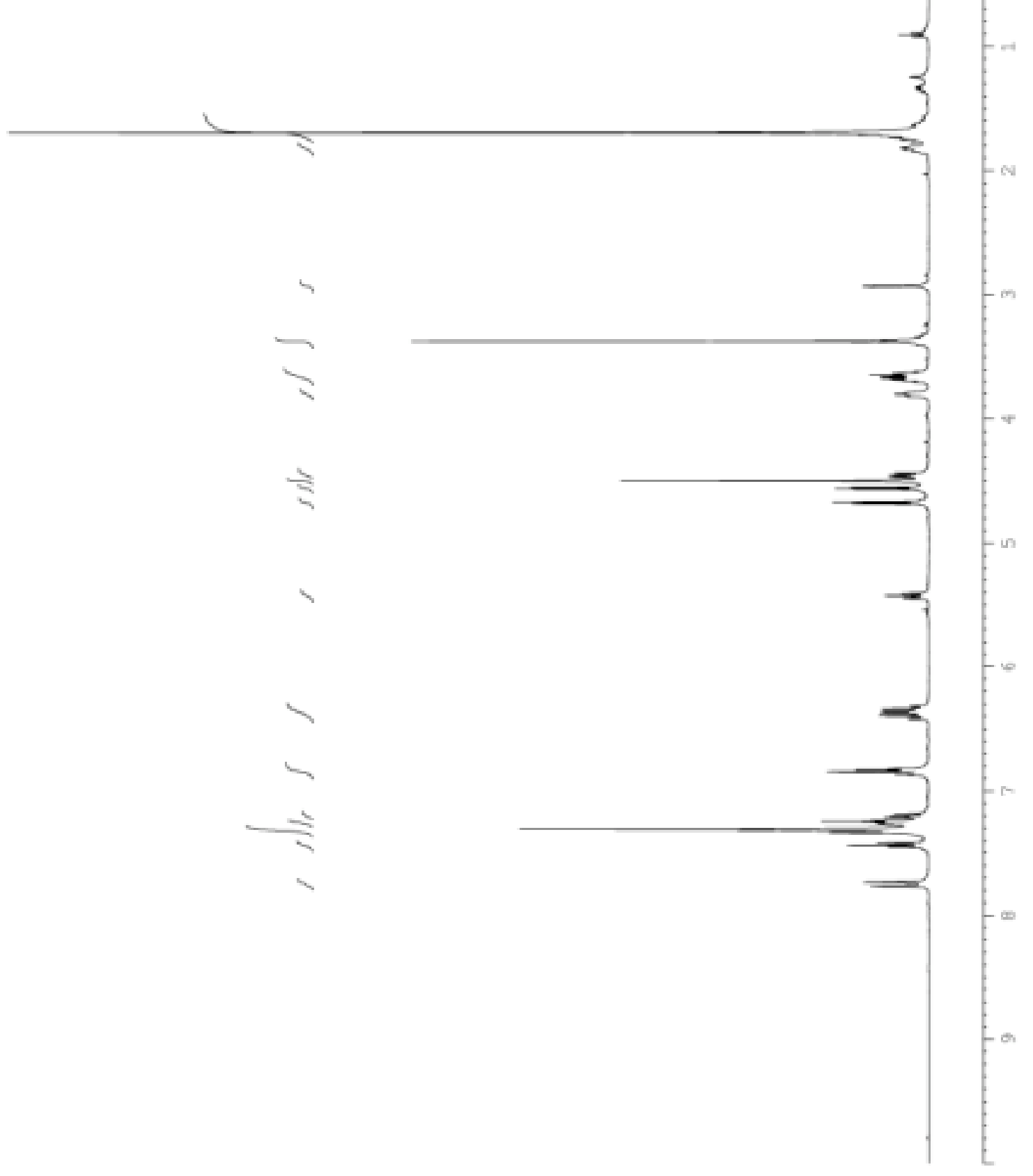



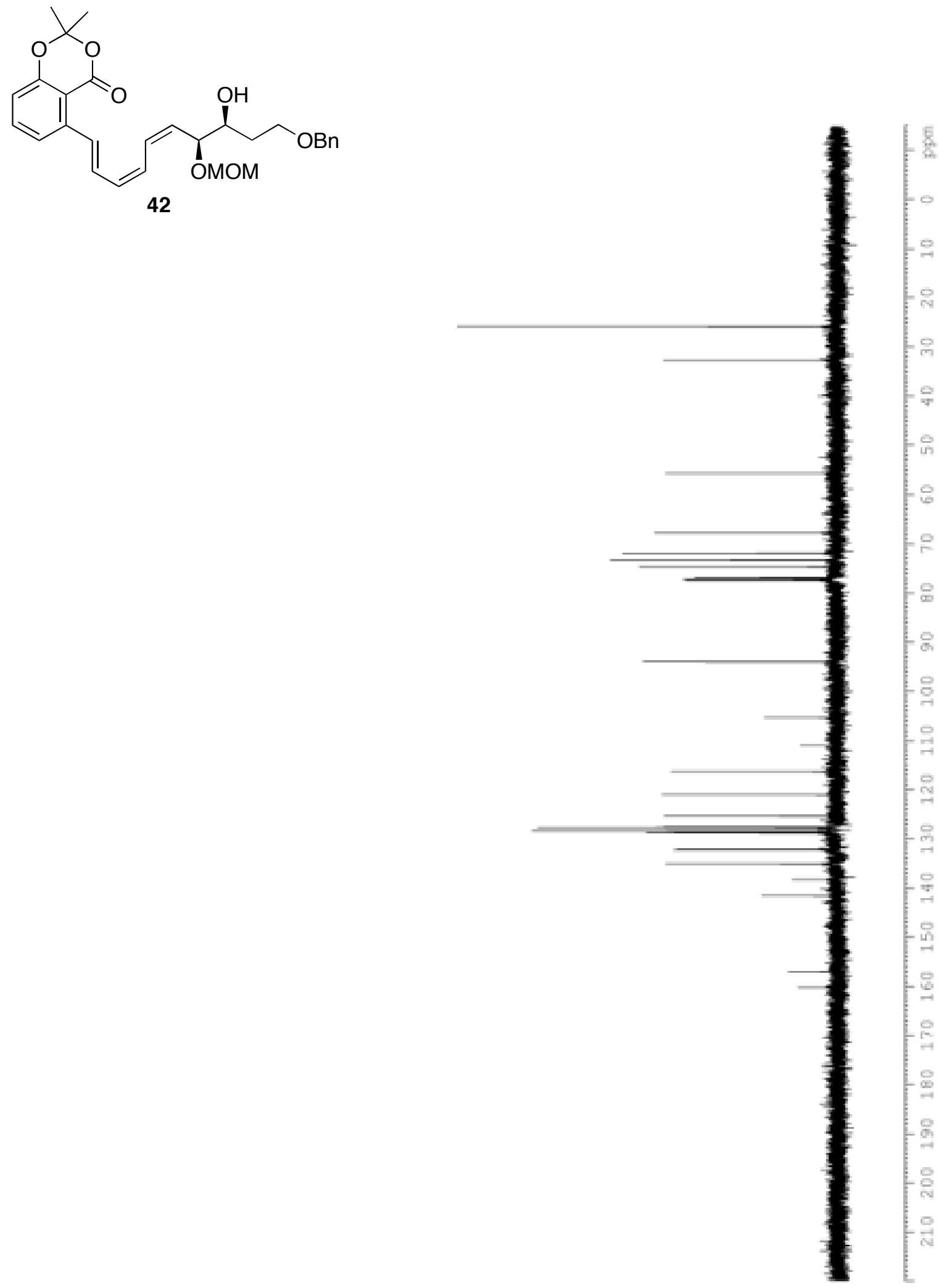


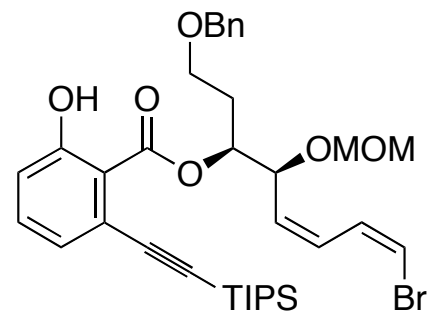

47

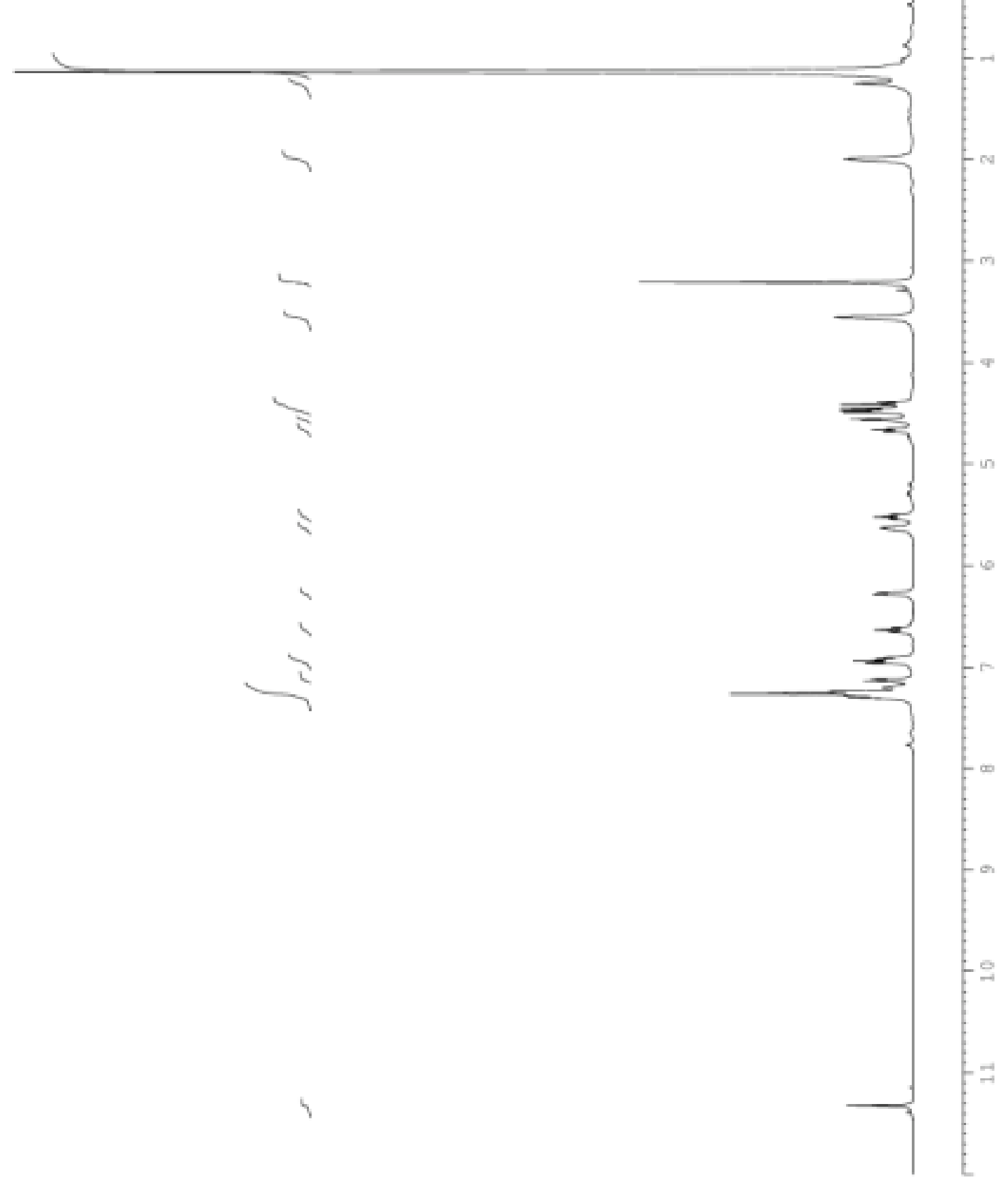



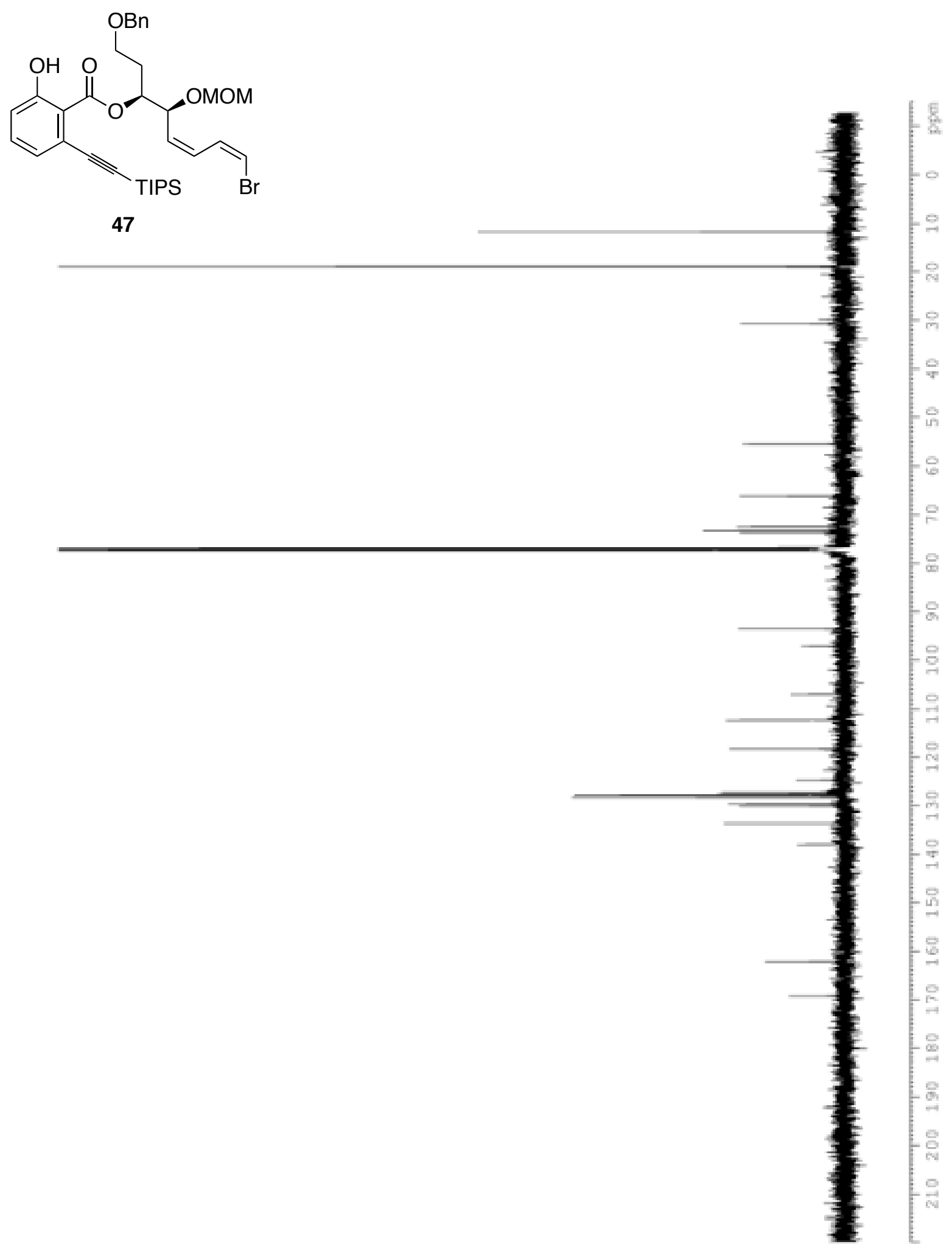


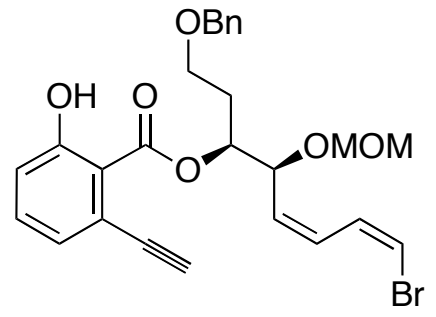

48
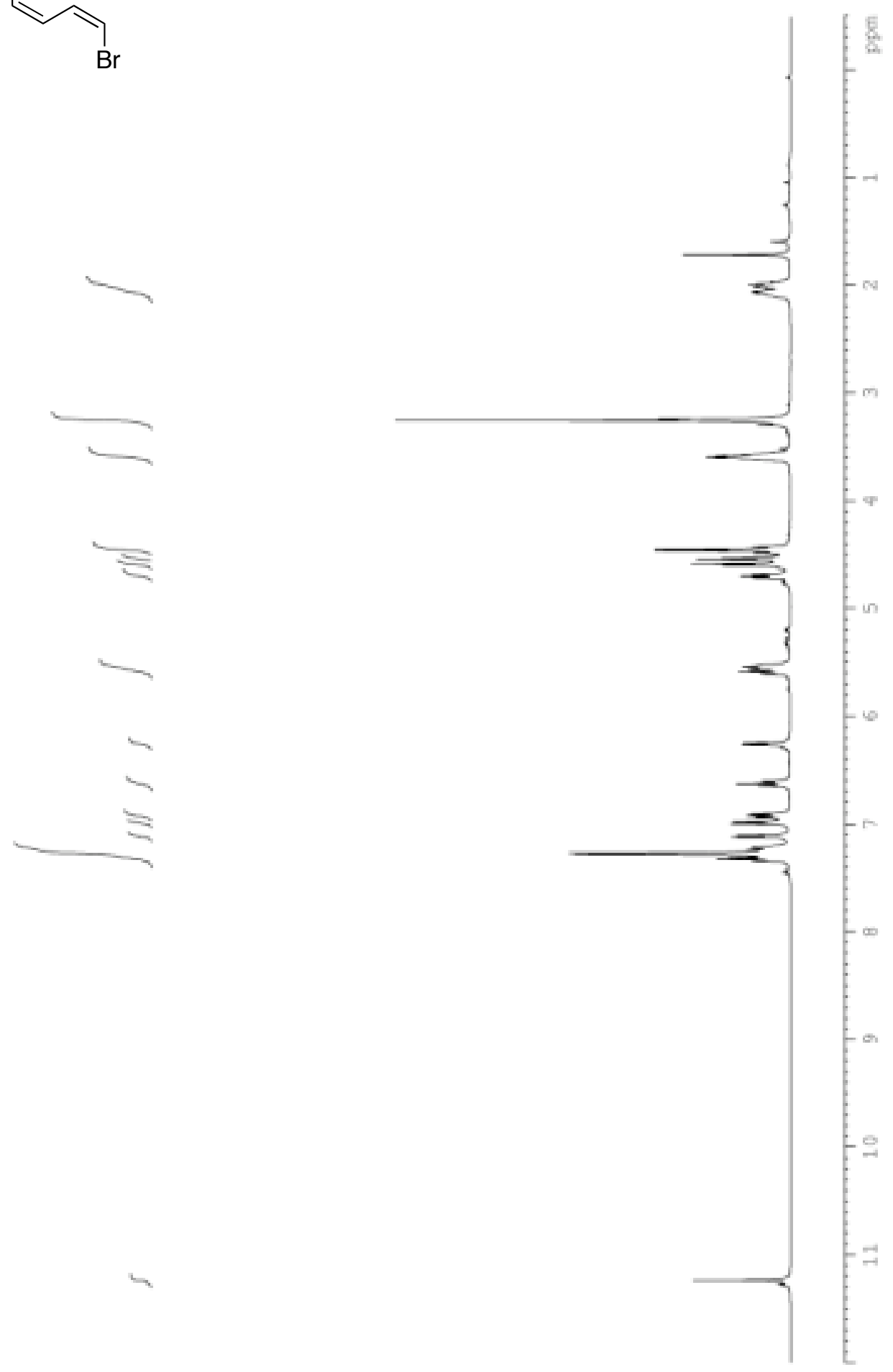


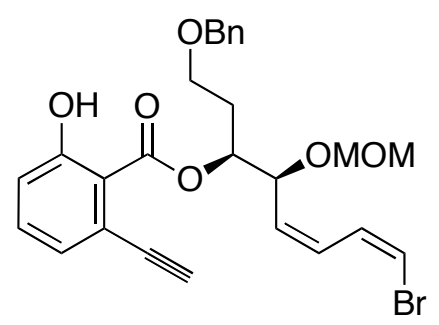

48

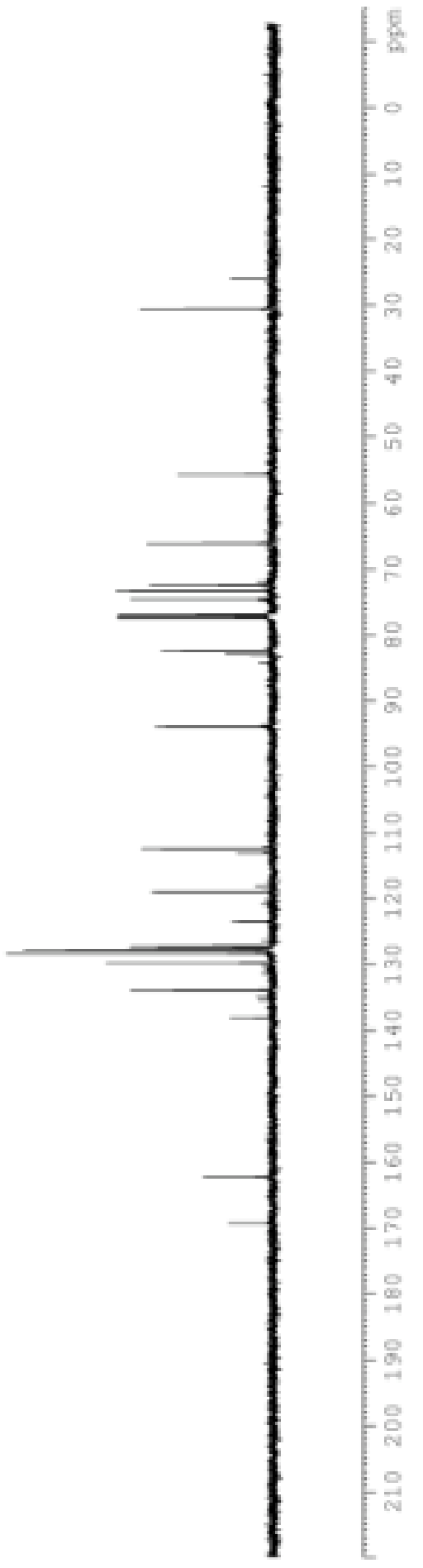




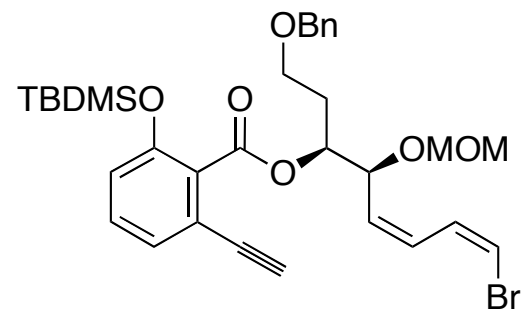

28

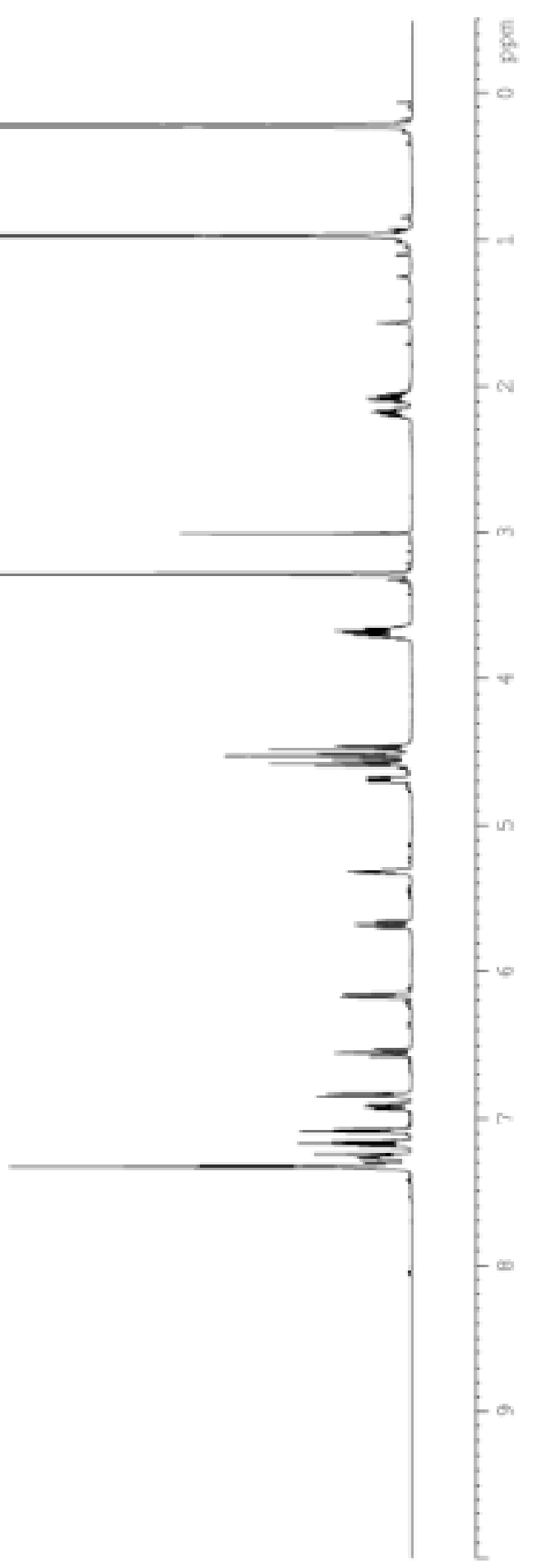




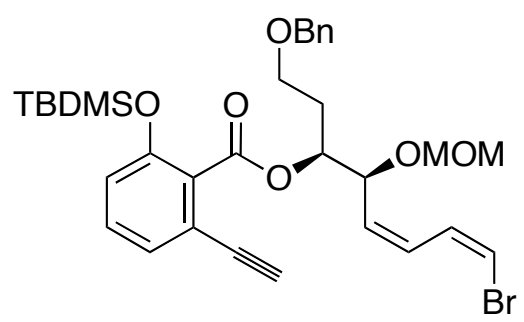

28

$\mathrm{Br}$

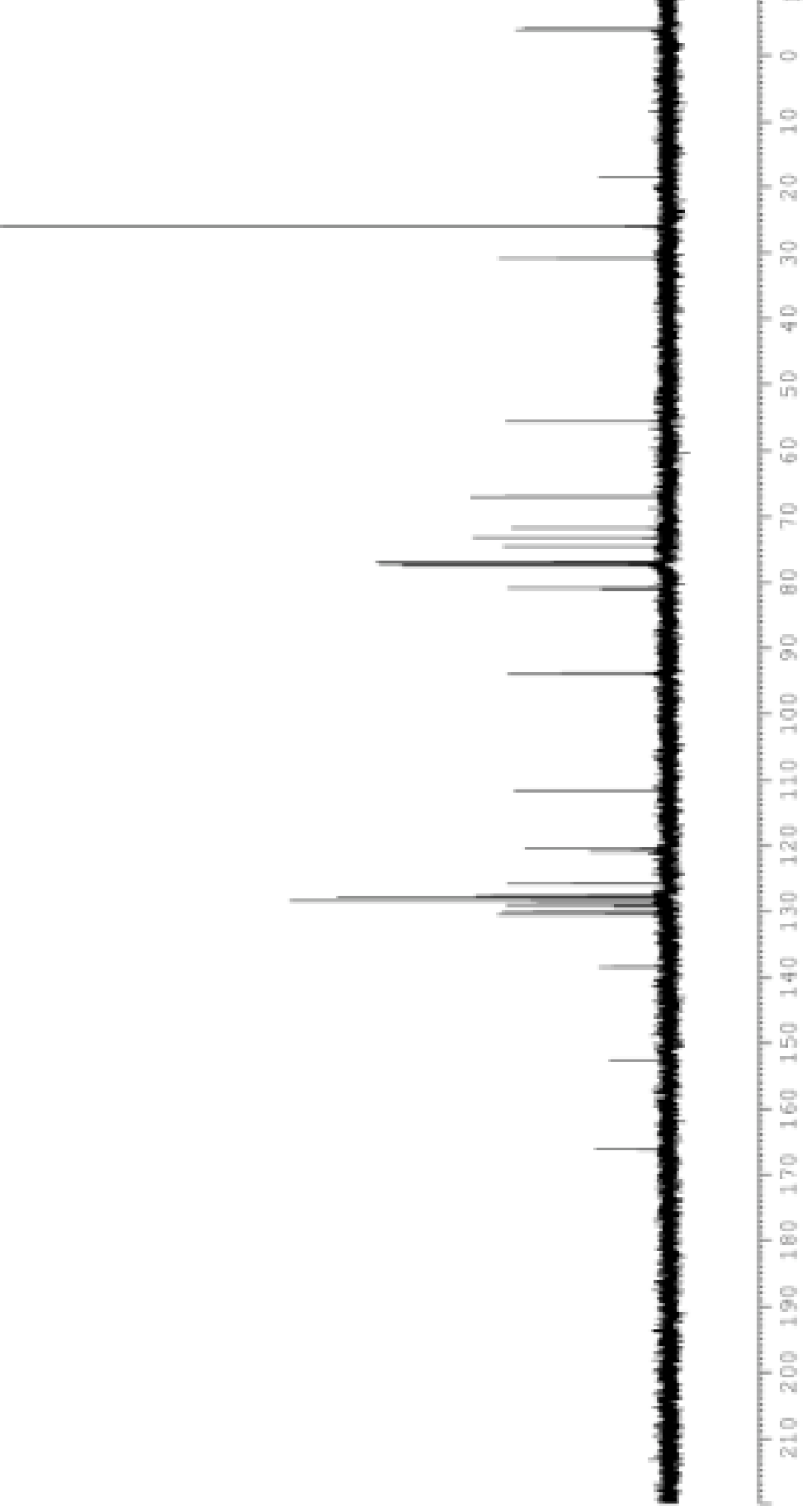




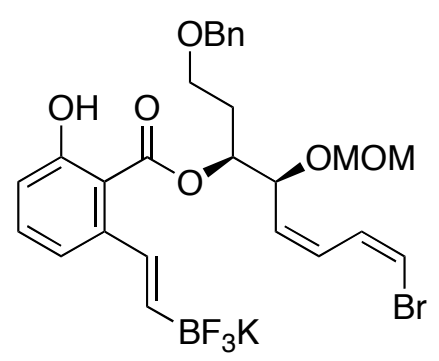

27

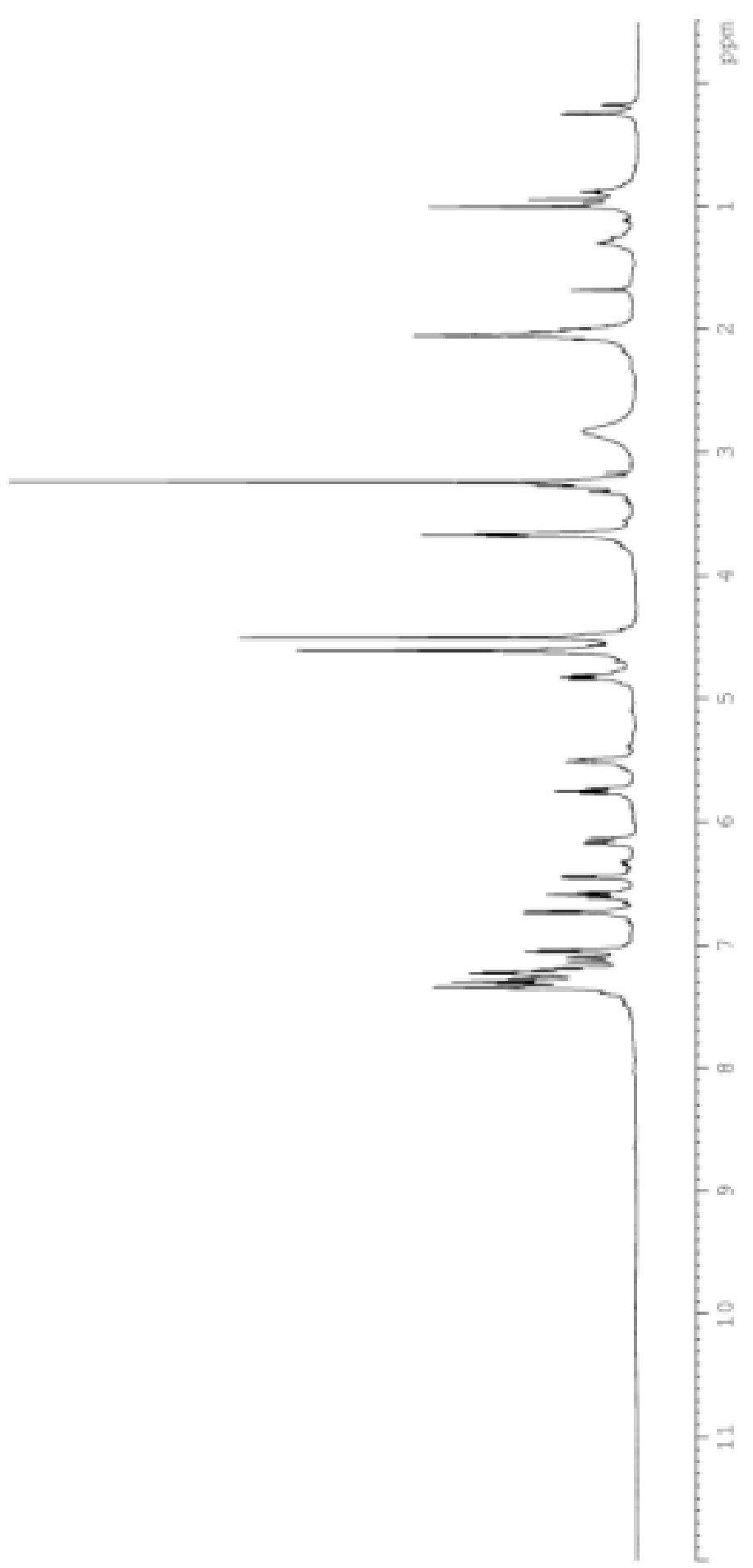




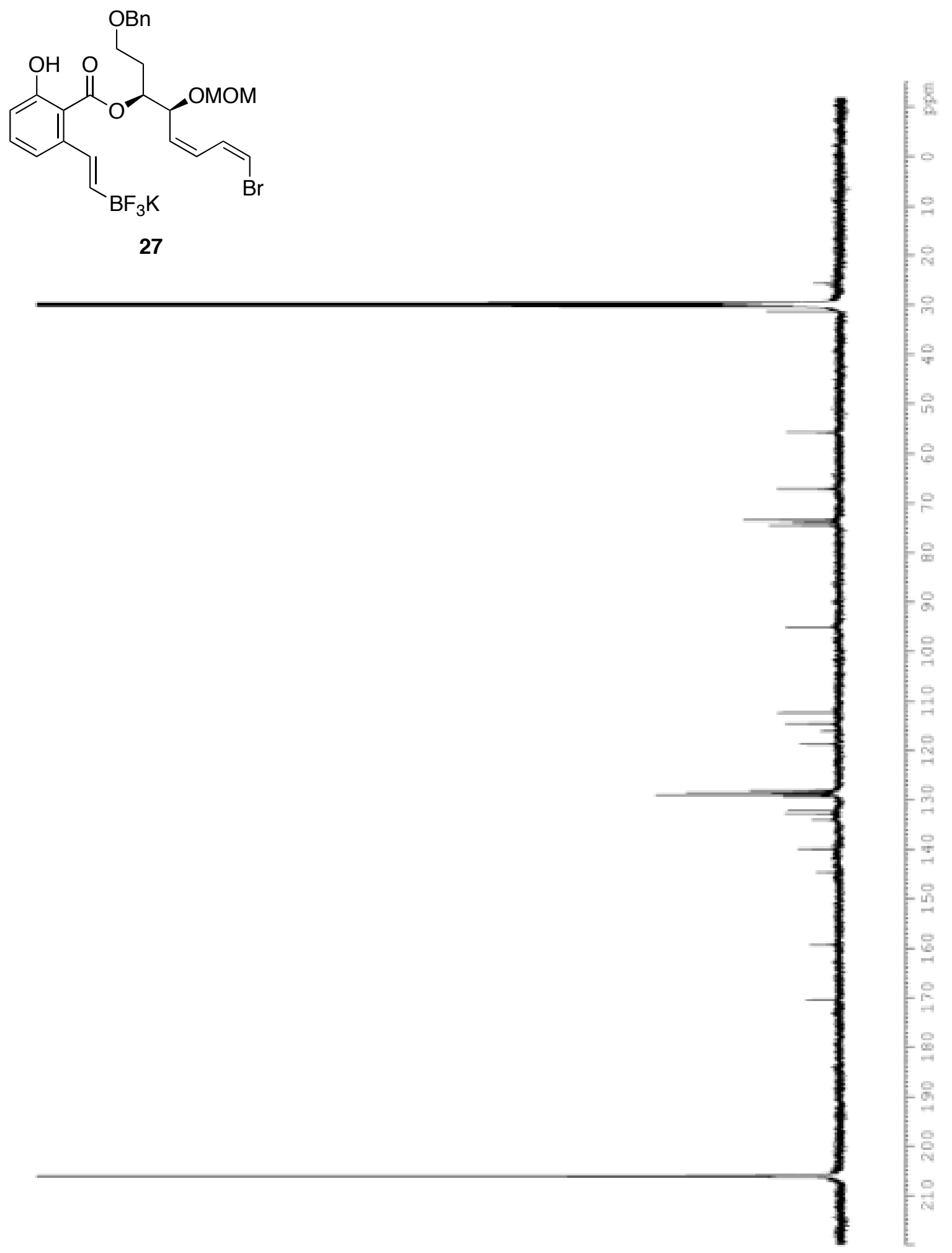

S65 

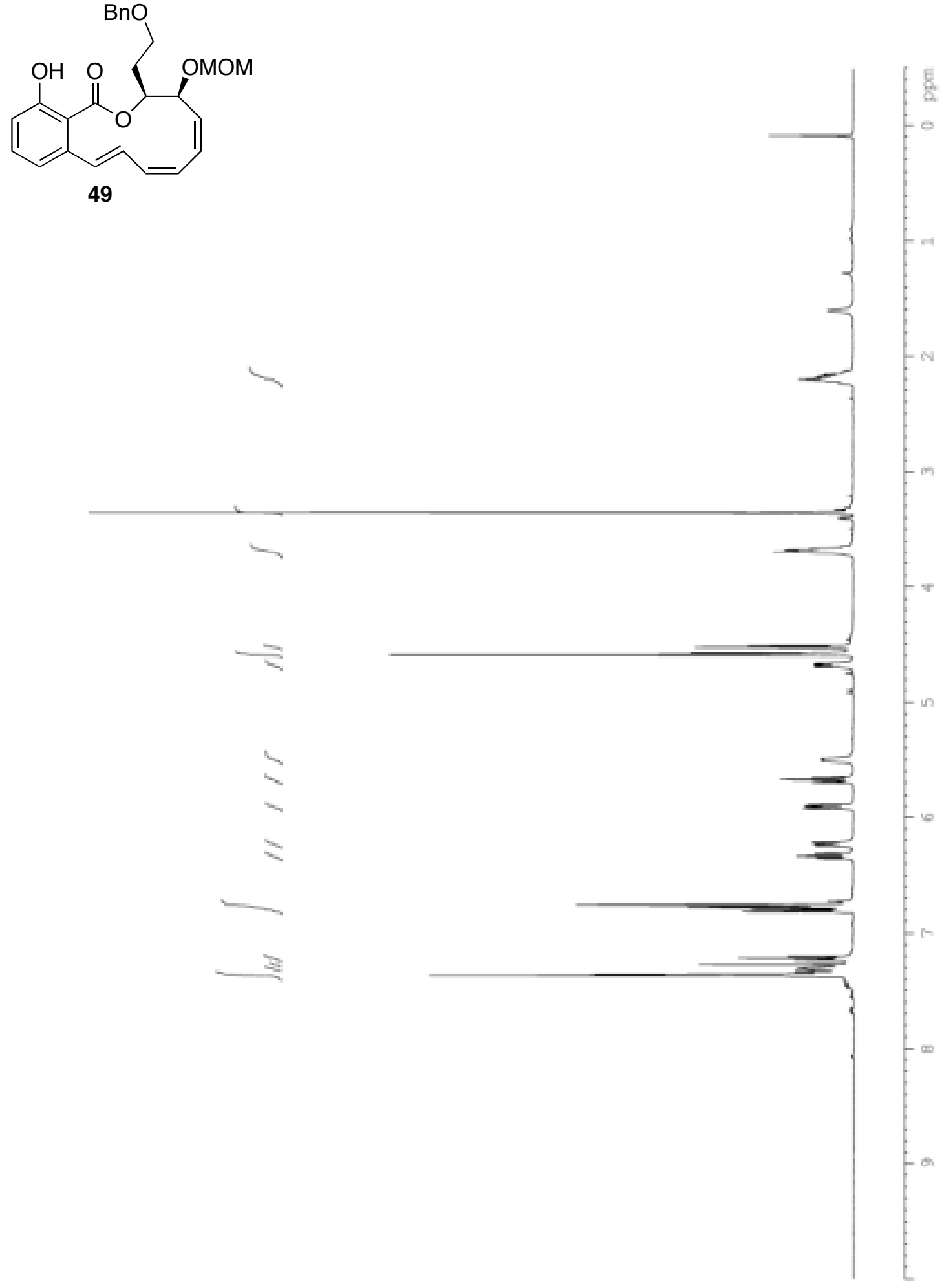

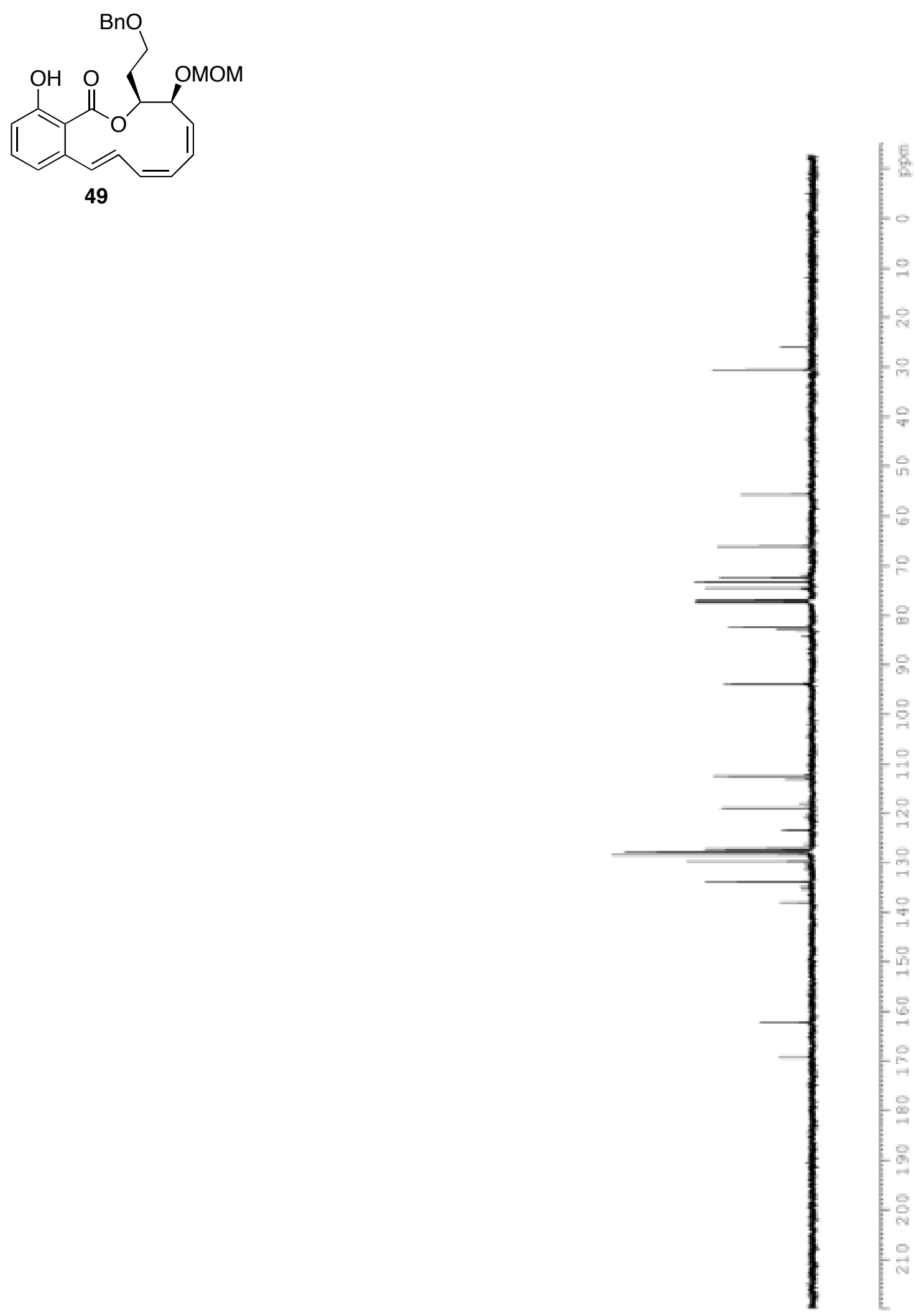

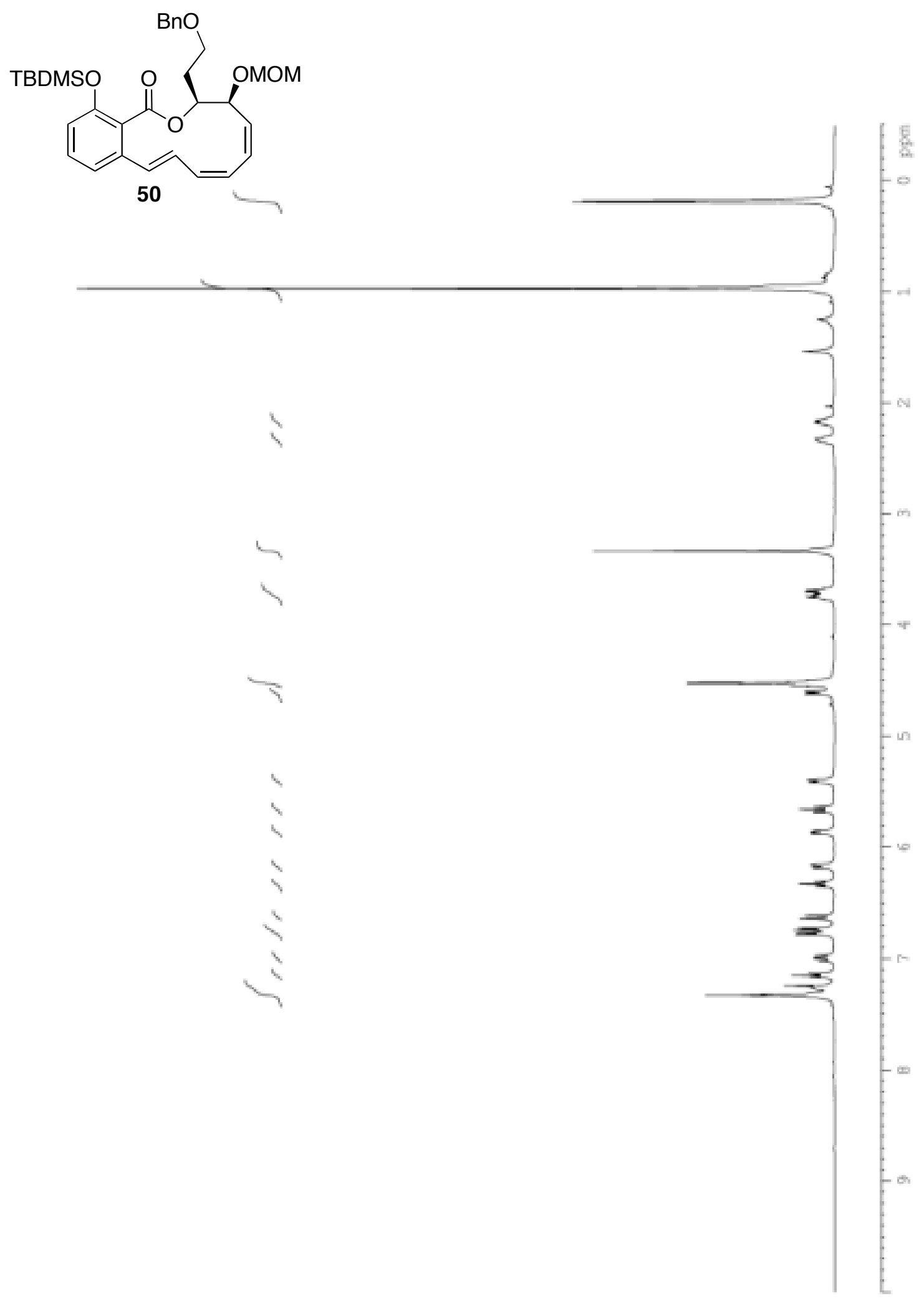

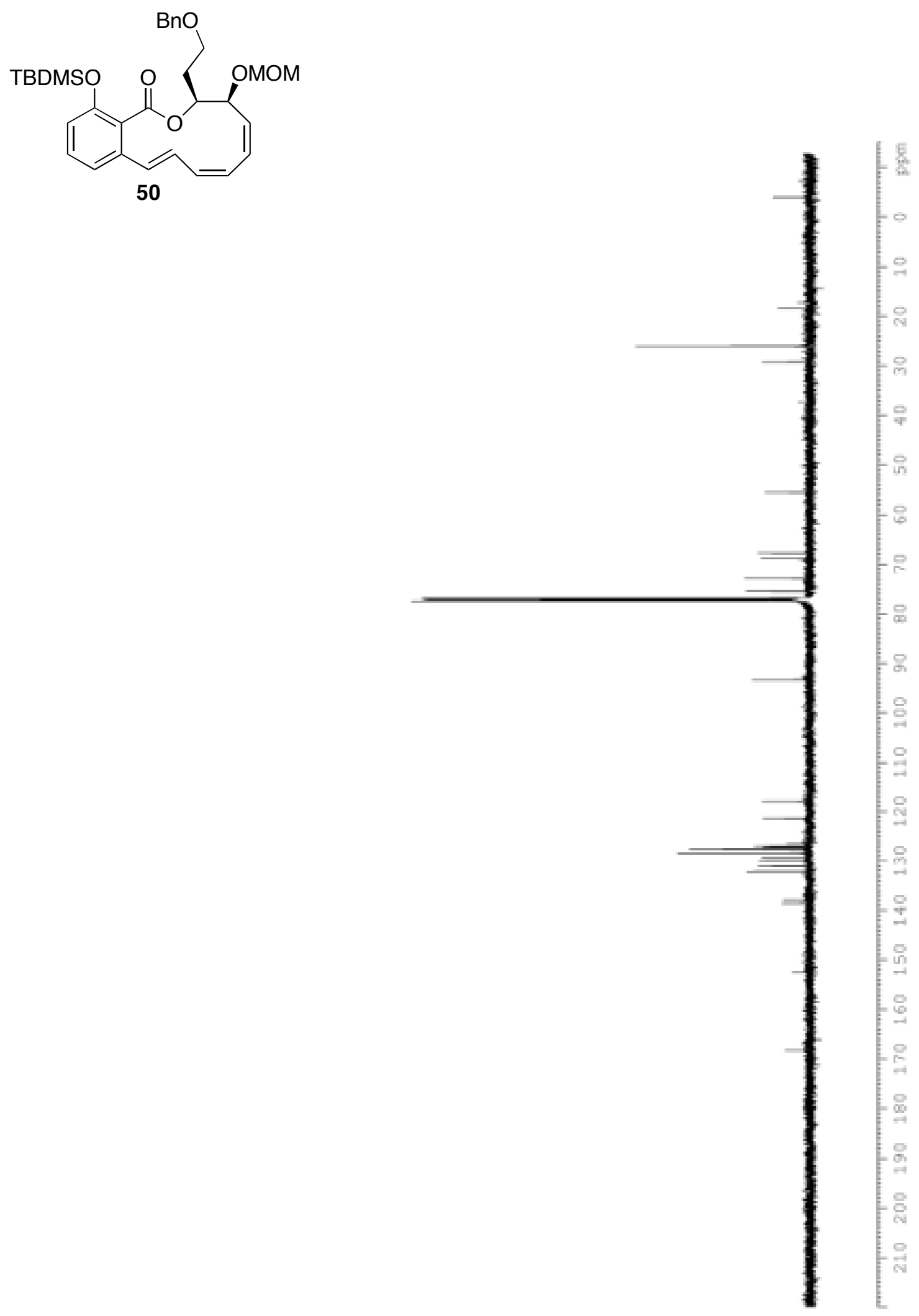

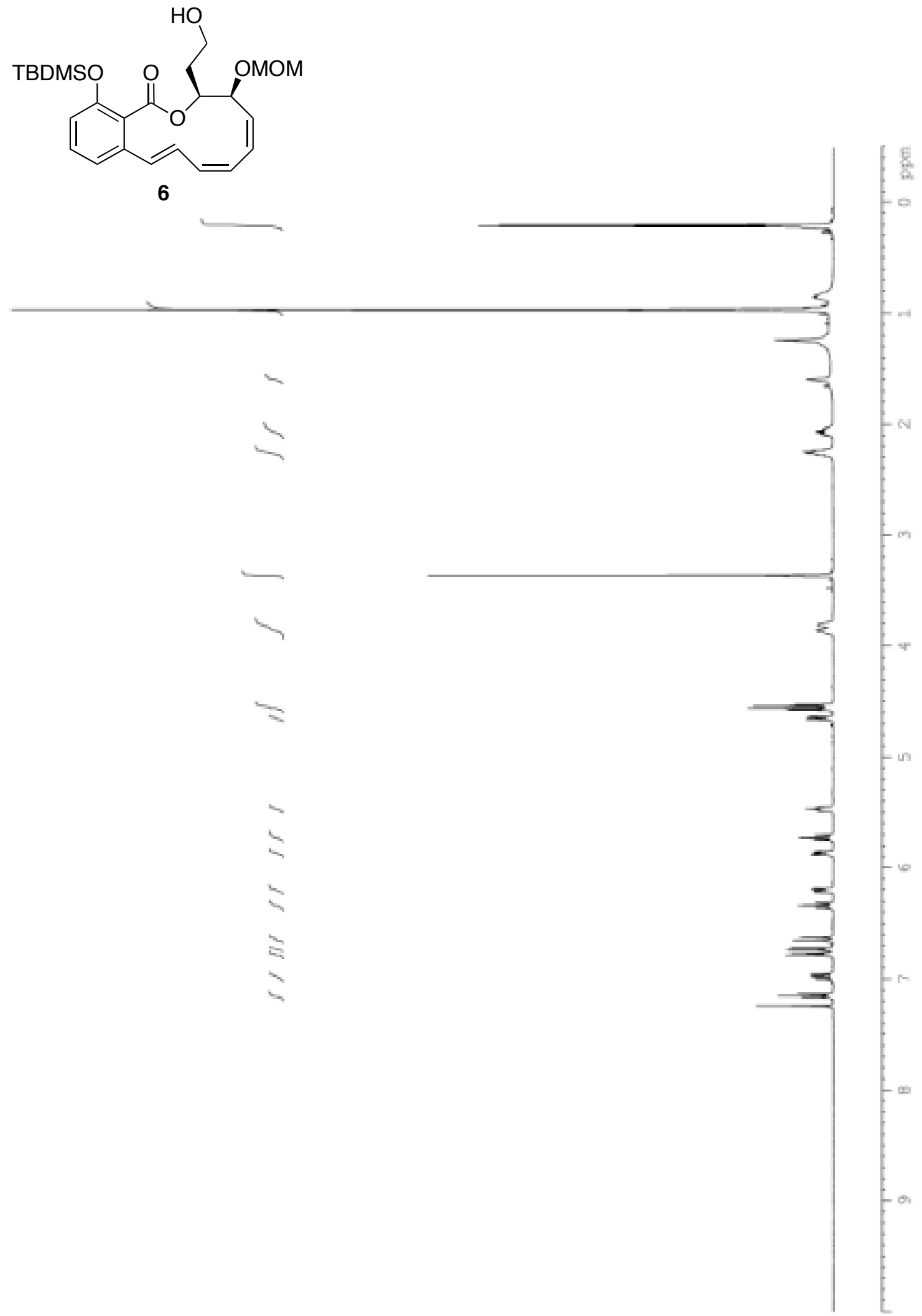

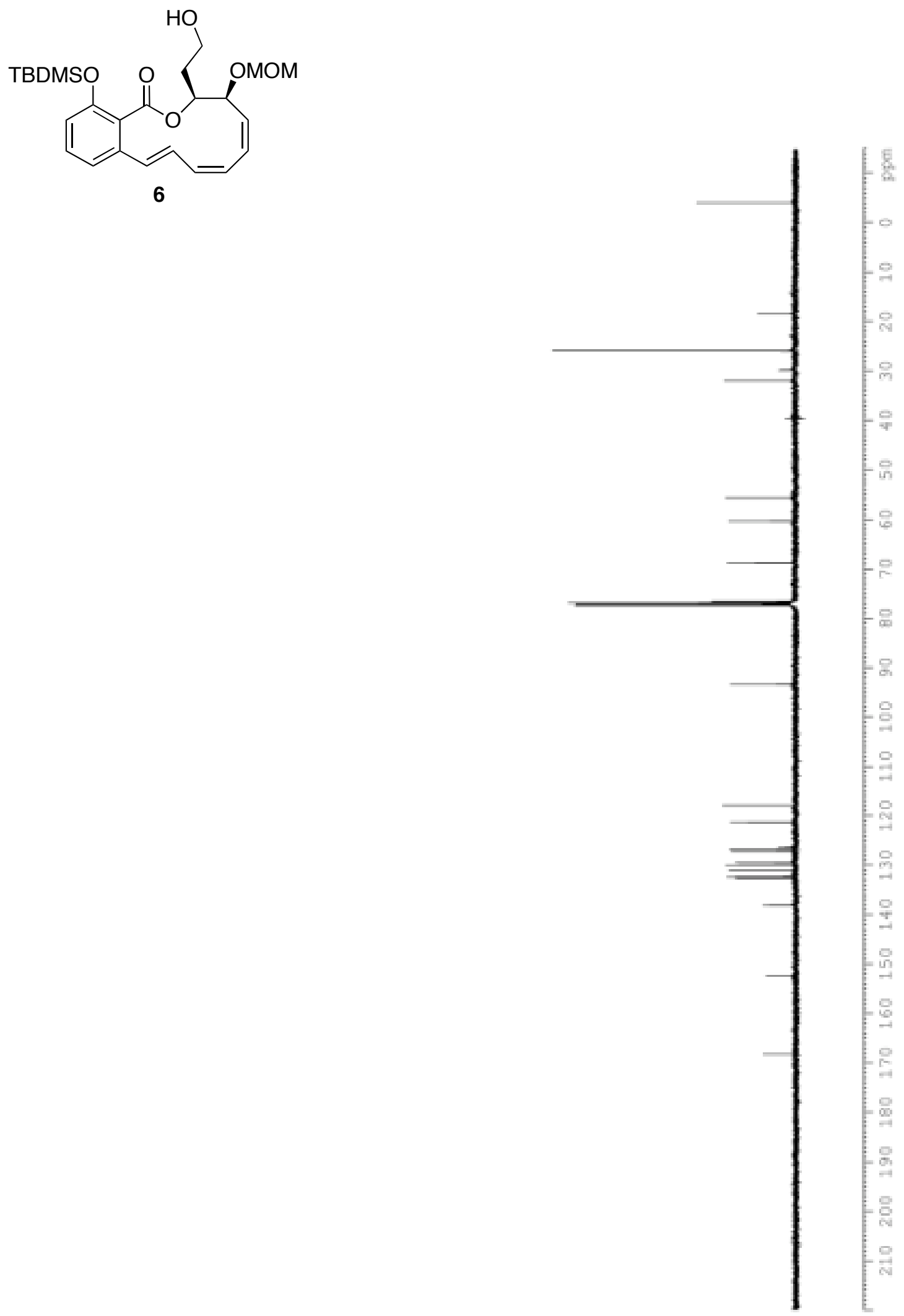